\title{
LOOP PRODUCTS AND CLOSED GEODESICS
}

\author{
MARK GORESKY ${ }^{1}$ AND NANCY HINGSTON ${ }^{2}$
}

\begin{abstract}
The critical points of the length function on the free loop space $\Lambda(M)$ of a compact Riemannian manifold $M$ are the closed geodesics on $M$. The length function gives a filtration of the homology of $\Lambda(M)$ and we show that the Chas-Sullivan product

$$
H_{i}(\Lambda) \times H_{j}(\Lambda) \stackrel{*}{\rightarrow} H_{i+j-n}(\Lambda)
$$

is compatible with this filtration. We obtain a very simple expression for the associated graded homology ring $\operatorname{GrH}_{*}(\Lambda(M))$ when all geodesics are closed, or when all geodesics are nondegenerate. We also construct a new but related cohomology product

$$
H^{i}\left(\Lambda, \Lambda_{0}\right) \times H^{j}\left(\Lambda, \Lambda_{0}\right) \stackrel{\circledast}{\rightarrow} H^{i+j+n-1}\left(\Lambda, \Lambda_{0}\right)
$$

(where $\Lambda_{0}=M$ is the constant loops), also compatible with the length filtration, and we obtain a similar expression for the ring $\operatorname{Gr} H^{*}\left(\Lambda, \Lambda_{0}\right)$ in these two cases. The non-vanishing of products $\sigma^{* n} \in H_{*}(\Lambda)$ and $\tau^{\circledast n} \in H^{*}\left(\Lambda, \Lambda_{0}\right)$ is shown to be related R. Bott's analysis of the rate at which the Morse index grows when a geodesic is iterated.
\end{abstract}

\section{CONTENTS}

1. Introduction

2. The free loop space

3. The finite dimensional approximation of Morse

4. Support, Critical values, and level homology

5. The Chas-Sullivan Product

6. Index growth

7. Level nilpotence

8. Cohomology products

9. Support and critical levels

10. Level nilpotence for cohomology

11. Level products in the nondegenerate case

12. Homology product when all geodesics are closed

13. Cohomology products when all geodesics are closed

14. Related products

Appendix A. Čech homology and cohomology

Appendix B. Thom isomorphisms

Appendix C. Theorems of Morse and Bott

Appendix D. Proof of Theorem 14.2

Appendix E. Associativity of $\circledast$

References

Key words and phrases. Chas-Sullivan product, loop product, free loop space, Morse theory, energy. 1. School of Mathematics, Institute for Advanced Study, Princeton N.J. Research partially supported by DARPA grant \# HR0011-04-1-0031.

2. Dept. of Mathematics, College of New Jersey, Ewing N.J.. 


\section{Introduction}

1.1. Let $M$ be a smooth compact manifold without boundary. In [CS], M. Chas and D. Sullivan constructed a new product structure

$$
H_{i}(\Lambda) \times H_{j}(\Lambda) \stackrel{*}{\longrightarrow} H_{i+j-n}(\Lambda)
$$

on the homology $H_{*}(\Lambda)$ of the free loop space $\Lambda$ of $M$. In CKS it was shown that this product is a homotopy invariant of the underlying manifold $M$. In contrast, the closed geodesics on $M$ depend on the choice of a Riemannian metric, which we now fix. In this paper we investigate the interaction beween the Chas-Sullivan product on $\Lambda$ and the energy function, or rather, its square root,

$$
F(\alpha)=\sqrt{E(\alpha)}=\left(\int_{0}^{1}\left|\alpha^{\prime}(t)\right|^{2} d t\right)^{1 / 2},
$$

whose critical points are exactly the closed geodesics. For any $a, 0 \leq a \leq \infty$ we denote by

$$
\Lambda^{\leq a}, \Lambda^{>a}, \Lambda^{=a}, \Lambda^{(a, b]}
$$

those loops $\alpha \in \Lambda$ such that $F(\alpha) \leq a, F(\alpha)>a, F(\alpha)=a, a<F(\alpha) \leq b$, etc. (When $a=\infty$ we set $\Lambda^{<a}=\Lambda^{\leq a}=\Lambda$.) In this paper we will use homology $H_{*}\left(\Lambda^{\leq a} ; G\right)$ with coefficients in the ring $G=\mathbb{Z}$ if $M$ is orientable and $G=\mathbb{Z} /(2)$ otherwise. In $\$ 5$ we prove the following.

1.2. Theorem. The Chas-Sullivan product extends to a family of product 1

$$
\begin{gathered}
\check{H}_{i}\left(\Lambda^{\leq a}\right) \times \check{H}_{j}\left(\Lambda^{\leq b}\right) \stackrel{*}{\longrightarrow} \check{H}_{i+j-n}\left(\Lambda^{\leq a+b}\right) \\
\check{H}_{i}\left(\Lambda^{\leq a}, \Lambda^{\leq a^{\prime}}\right) \times \check{H}_{j}\left(\Lambda^{\leq b}, \Lambda^{\leq b^{\prime}}\right) \stackrel{*}{\longrightarrow} \check{H}_{i+j-n}\left(\Lambda^{\leq a+b}, \Lambda^{\leq \max \left(a+b^{\prime}, a^{\prime}+b\right)}\right) \\
\check{H}_{i}\left(\Lambda^{\leq a}, \Lambda^{<a}\right) \times \check{H}_{j}\left(\Lambda^{\leq b}, \Lambda^{<b}\right) \stackrel{*}{\longrightarrow} \check{H}_{i+j-n}\left(\Lambda^{\leq a+b}, \Lambda^{<a+b}\right)
\end{gathered}
$$

whenever $0 \leq a^{\prime}<a \leq \infty$ and $0 \leq b^{\prime}<b \leq \infty$. These products are compatible with respect to the natural inclusions $\Lambda^{\leq c^{\prime}} \rightarrow \Lambda^{\leq c}$ whenever $c^{\prime} \leq c$.

We refer to $\check{H}_{i}\left(\Lambda^{\leq a}, \Lambda^{<a}\right)$ as the level homology group, or the homology at level $a$, with its associated level homology product (1.2.1). It is zero unless $a$ is a critical value of $F$.

1.3. In 98 we consider analogous products in the cohomology of the free loop space. (We discuss the cup product briefly in $\$ 9.4$.) It is possible to mimic the construction of the Chas-Sullivan product, word for word, in cohomology, but this results in a trivial product, cf. $\$ 8.1$. However by utilizing a certain one parameter family of reparametrizations, it is possible to construct a nontrivial product in cohomology.

\footnotetext{
${ }^{1}$ Here, $\check{H}_{i}(\Lambda \leq a)$ denotes Čech homology. In Lemma A.4 we show that the singular and Čech homology agree if $0 \leq a \leq \infty$ is a regular value or if it is a nondegenerate critical value in the sense of Bott.
} 
1.4. Theorem. Let $0 \leq a^{\prime}<a \leq \infty$ and $0 \leq b^{\prime}<b<\infty$. There is a family of products

$$
\begin{aligned}
& H^{i}\left(\Lambda, \Lambda_{0}\right) \times H^{j}\left(\Lambda, \Lambda_{0}\right) \stackrel{\circledast}{\longrightarrow} H^{i+j+n-1}\left(\Lambda, \Lambda_{0}\right) \\
& \check{H}^{i}\left(\Lambda^{\leq a}, \Lambda^{\leq a^{\prime}}\right) \times \check{H}^{j}\left(\Lambda^{\leq b}, \Lambda^{\leq b^{\prime}}\right) \stackrel{\circledast}{\longrightarrow} \check{H}^{i+j+n-1}\left(\Lambda^{\leq \min \left(a+b^{\prime}, a^{\prime}+b\right)}, \Lambda^{\leq a^{\prime}+b^{\prime}}\right) \\
& \check{H}^{i}\left(\Lambda^{\leq a}, \Lambda^{<a}\right) \times \check{H}^{j}\left(\Lambda^{\leq b}, \Lambda^{<b}\right) \stackrel{\circledast}{\longrightarrow} \check{H}^{i+j+n-1}\left(\Lambda^{\leq a+b}, \Lambda^{<a+b}\right)
\end{aligned}
$$

which are associative and (sign-)commutative, and are compatible with the homomorphisms induced by the inclusions $\Lambda^{\leq c^{\prime}} \rightarrow \Lambda^{\leq c}$ whenever $c^{\prime}<c$. The product (1.4.1) is independent of the Riemannian metric.

The same construction gives (cf. 98.4) a (possibly noncommutative) product $\circledast$ on the cohomology of the based loop space $\Omega$ such that $h^{*}(a \circledast b)=h^{*}(a) \circledast h^{*}(b)$ where $a, b \in H^{*}(\Lambda)$ and $h: \Omega \rightarrow \Lambda$ denotes the inclusion. In $\$ 13.9$ we calculate some non-zero examples of this product.

1.5. If the ring $\left(H^{*}\left(\Lambda, \Lambda_{0}\right), \circledast\right)$ is finitely generated then the existence of the product $\circledast$ is already enough to answer a question of Y. Eliashberg, cf. 99.5 , the maximal degree of an "essential" homology class of level $\leq t$ can grow at most linearly with $t$.

1.6. There is a well-known isomorphism between the Floer homology of the cotangent bundle of $M$ and the homology of the free loop space of $M$, which transforms the pair-ofpants product into the Chas-Sullivan product on homology, see AS1, AS2, SaW, Vi, CHV]. The cohomology product described above should therefore correspond to some geometrically defined product on the Floer cohomology; it would be interesting to see an explicit construction of this product. (The obvious candidate would be some 1-parameter variation of the coproduct on homology given by the upside-down pair of pants.) It would also be interesting to compare the cohomology product described above with the coproduct in homology that is outlined in $[\mathrm{Su}]$. (For odd dimensional spheres the product in [Su is zero while the $\circledast$ product is non-zero.)

1.7. The critical value (see 94 ) of a homology class $0 \neq \eta \in H_{i}(\Lambda)$ is defined to be

$$
\operatorname{cr}(\eta)=\inf \left\{a \in \mathbb{R}: \eta \text { is supported on } \Lambda^{\leq a}\right\} .
$$

The critical value of a cohomology class $0 \neq \alpha \in H^{*}\left(\Lambda, \Lambda_{0}\right)$ is defined to be

$$
\operatorname{cr}(\alpha)=\sup \left\{a \in \mathbb{R}: \quad \alpha \text { is supported on } \Lambda^{\geq a}\right\} .
$$

(These are necessarily critical values of $F$.) In Proposition 5.4 and Proposition 9.2 we show that the products $*$ and $\circledast$ satisfy the following relations:

$$
\begin{aligned}
& \operatorname{cr}(\alpha * \beta) \leq \operatorname{cr}(\alpha)+\operatorname{cr}(\beta) \text { for all } \alpha, \beta \in H_{*}(\Lambda) \\
& \operatorname{cr}(\alpha \circledast \beta) \geq \operatorname{cr}(\alpha)+\operatorname{cr}(\beta) \text { for all } \alpha, \beta \in H^{*}\left(\Lambda, \Lambda_{0}\right) .
\end{aligned}
$$


1.8. A homology class $\eta \in H_{*}(\Lambda)$ is said to be level nilpotent if $\operatorname{cr}\left(\eta^{* N}\right)<N \operatorname{cr}(\eta)$ for some $N>1$. A cohomology class $\alpha \in H^{*}\left(\Lambda, \Lambda_{0}\right)$ is level nilpotent if $\operatorname{cr}\left(\alpha^{\circledast N}\right)>N \operatorname{cr}(\alpha)$ for some $N>1$. There are analogous notions in level homology and cohomology: A homology (resp. cohomology) class $\eta$ in $\check{H}\left(\Lambda^{\leq a}, \Lambda^{<a}\right.$ ) (where $\check{H}$ denotes homology, resp. cohomology) is said to be level-nilpotent if some power vanishes: $\eta^{* N}=0$ (resp. $\left.\eta^{\circledast N}=0\right)$ in $\left.\check{H}_{(} \Lambda^{\leq N a}, \Lambda^{<N a}\right)$. In $\$ 7$ and $\$ 10$ we prove:

1.9. Theorem. If all closed geodesics on $M$ are nondegenerate then every homology class in $H_{*}(\Lambda)$, every cohomology class in $H^{*}\left(\Lambda, \Lambda_{0}\right)$, every level homology class and every level cohomology clas $\Phi^{2}$ in $H\left(\Lambda^{\leq a}, \Lambda^{<a}\right)$ is level-nilpotent (for all $a \in \mathbb{R}$ ).

1.10. On the other hand, non-nilpotent classes exist when all geodesics are closed. Suppose $E$ is the energy function of a metric in which all geodesics on $M$ are closed, simply periodic, and have the same prime length $\ell$, as defined in \$12.1. The critical values of $F=\sqrt{E}$ are the (non-negative) integer multiples of $\ell$. The set of critical points with critical value $r \ell(r \geq 1)$ form a (Morse-Bott) nondegenerate critical submanifold $\Sigma_{r} \subset \Lambda$ that is diffeomorphic to the unit sphere bundle $S M$ by the mapping $\alpha \mapsto \alpha^{\prime}(0) / r \ell$.

Let $\lambda_{r}$ be the Morse index of any geodesic of length $r \ell$. Let $h=\lambda_{1}+2 n-1$ where $n=\operatorname{dim}(M)$. Then $H_{i}(\Lambda \leq \ell)=0$ for $i>h$. Let

$$
\Theta \in H_{h}\left(\Lambda^{\leq \ell} ; G\right) \cong G
$$

be a generator of the top degree homology group. In \$12 and Corollary 12.7 we prove:

\subsection{Theorem. The $r$-fold Chas-Sullivan product}

$$
\Theta^{* r} \in H_{\lambda_{r}+2 n-1}\left(\Lambda^{\leq r \ell}, \Lambda^{<r \ell} ; G\right) \cong G
$$

generates the top degree homology at the level rl, and more generally, the Chas-Sullivan product with $\Theta$ induces an isomorphism

$$
H_{i}\left(\Lambda^{\leq a}, \Lambda^{<a}\right) \rightarrow H_{i+h-n}\left(\Lambda^{\leq a+\ell}, \Lambda^{<a+\ell}\right)
$$

for all degrees $i$ and for all level values a. The energy $E$ determines a filtration $0=I_{0} \subset$ $I_{1} \subset \cdots \subset H_{*}\left(\Lambda, \Lambda_{0}\right)$ such that $I_{j} * I_{k} \subset I_{j+k}$. The associated graded ring is isomorphic (with degree shifts) to the ring

$$
G r_{I} H_{*}\left(\Lambda, \Lambda_{0}\right) \cong H_{*}(S M)[T]_{\geq 1}
$$

of polynomials of degree $\geq 1$, where $H_{*}(S M)$ denotes the homology (intersection) ring of $S M$.

The full Chas-Sullivan ring $H_{*}(\Lambda)$ was computed by R. Cohen, J. Jones, and J. Yan CJY] for spheres and projective spaces. The relatively simple formula (1.11.1) is compatible with their computation. It seems likely that there may be other results along these lines when the Riemannian metric has large sets of closed geodesics.

\footnotetext{
${ }^{2}$ see previous footnote
} 
1.12. In \$13 and Corollary [13.8 we prove the analogous result for cohomology. Suppose that all geodesics on $M$ are closed, simply periodic, and have the same prime length $\ell$. Then $H^{i}\left(\Lambda^{\leq \ell}, \Lambda^{=0}\right)=0$ for $i<\lambda_{1}$. Let

$$
\Omega \in H^{\lambda_{1}}\left(\Lambda^{\leq \ell}, \Lambda^{=0} ; G\right) \cong G
$$

be a generator of the lowest degree cohomology group $G=\mathbb{Z}$ or $\mathbb{Z} /(2)$.

1.13. Theorem. The r-fold product

$$
\Omega^{\circledast r} \in H^{\lambda_{r}}\left(\Lambda^{\leq r \ell}, \Lambda^{<r \ell}\right) \cong G
$$

generates the lowest degree cohomology class at level $r \ell$ and more generally, the product with $\Omega$ induces an isomorphism

$$
H^{i}\left(\Lambda^{\leq a}, \Lambda^{<a}\right) \rightarrow H^{i+h-n}\left(\Lambda^{\leq a+\ell}, \Lambda^{<a+\ell}\right)
$$

for all degrees $i$ and all level values a. Moreover, the energy induces a filtration

$$
H^{i}\left(\Lambda, \Lambda_{0}\right)=I^{0} \supset I^{1} \supset I^{2} \supset \cdots
$$

by ideals such that $I^{j} \circledast I^{k} \subset I^{j+k}$. The associated graded ring $\operatorname{Gr}^{I} H^{*}\left(\Lambda, \Lambda_{0}\right)$ is isomorphic (with degree shifts) to the ring,

$$
H^{*}(S M)[T]_{\geq 1}
$$

where $H^{*}(S M)$ denotes the cohomology ring of $S M$.

1.14. Counting closed geodesics. By VS if $M$ is a compact, simply connected Riemannian manifold whose cohomology algebra $H^{*}(M ; \mathbb{Q})$ cannot be generated by a single element then the Betti numbers of $\Lambda$ form an unbounded sequence, whence by [GrM], the manifold $M$ admits infinitely many prime closed geodesics. This result leaves open the case of spheres and projective spaces (among others).

It is known [Ban, $\left[\mathrm{F}\right.$, Hi2] that any Riemannian metric on $S^{2}$ has infinitely many prime closed geodesics, and it is conjectured that the same holds for any Riemannian sphere or projective space of dimension $n>2$. (But see [Z] for examples of Finsler metrics on $S^{2}$ with finitely many prime closed geodesics, all of which are nondegenerate.) It should, in principle, be possible to count the number of closed geodesics using Morse theory on the free loop space $\Lambda$, but each prime geodesic $\gamma$ is associated with infinitely many critical points, corresponding to the iterates $\gamma^{m}$. So it would be useful to have an operation on $H_{*}(\Lambda)$ that corresponds to the iteration of closed geodesics.

If $\lambda_{1}$ is the Morse index of a prime closed geodesic $\gamma$ of length $\ell$, then by Bo1] (cf. Proposition 6.1), the Morse index $\lambda_{m}$ of the iterate $\gamma^{m}$ can be anywhere between $m \lambda_{1}-$ $(m-1)(n-1)$ and $m \lambda_{1}+(m-1)(n-1)$. For nondegenerate critical points, the ChasSullivan product $[\bar{\gamma}] * \cdots *[\bar{\gamma}]$ is non-zero exactly when (cf. Theorem 11.3 ) the index growth is minimal (i.e., when $\left.\lambda_{m}=m \lambda_{1}-(m-1)(n-1)\right)$. Here, $[\bar{\gamma}] \in H_{\lambda_{1}+1}\left(\Lambda^{\leq \ell}, \Lambda^{<\ell}\right)$ is the level homology class represented by the $S^{1}$ saturation of $\gamma$. The Pontrjagin product (on the level 
homology of the based loop space) is zero unless $\lambda_{m}=m \lambda_{1}$. The level cohomology product $\circledast$ is non-zero when the index growth is maximal (cf. Proposition 6.1).

1.15. Related products. In [CS], Chas and Sullivan also defined a Lie algebra product $\{\alpha, \beta\}$ on the homology $H_{*}(\Lambda)$ of the free loop space. In $\$ 14$ we combine their ideas with the construction of the cohomology product $\circledast$ to produce a Lie algebra product on the cohomology $H^{*}\left(\Lambda, \Lambda_{0}\right)$ In $\$ 14.3$ we use the calculations described in $\$ 1.14$ to show that these products are sometimes non-zero. Also following [CS], we construct products on the $T=S^{1}$-equivariant cohomology $H_{T}^{*}\left(\Lambda, \Lambda_{0}\right)$.

1.16. Several of the proofs in this paper require technical results that are well-known to experts (in different fields) but are difficult to find in the literature. These technical tools are described in the Appendices, as are the (tedious) proofs of Proposition 8.3 and Theorem 14.2. The collection of products and their definitions can be rather confusing, so in each case we have created a "boxed" diagram which gives a concise way to think about the product.

1.17. Acknowledgments. We wish to thank Fred Cohen and Dennis Sullivan for a number of valuable conversations. We are pleased to thank Matthias Schwarz, who long ago pointed out to the second author the concept and importance of nilpotence of products, in the context of the pair-of-pants product. The authors would also like to thank the Institute for Advanced Study in Princeton N.J. for its hospitality and support during the academic year 2005-2006. The second author was supported in 2005-06 by a grant to the Institute for Advanced Study by the Bell Companies Fellowship. The first author is grateful to the Defense Advanced Research Projects Agency for its support from grant number HR0011-04-1-0031.

\section{The free loop space}

2.1. Throughout this paper, $M$ denotes an $n$ dimensional smooth connected compact Riemannian manifold. Let $\alpha:[a, b] \rightarrow M$ be a piecewise smooth curve. Its length and energy are given by

$$
L(\alpha)=\int_{a}^{b}\left|\alpha^{\prime}(t)\right| d t \text { and } E(\alpha)=\int_{a}^{b}\left|\alpha^{\prime}(t)\right|^{2} d t .
$$

The Cauchy-Schwartz inequality says that $L(\alpha)^{2} \leq(b-a) E(\alpha)$. The formulas work out most simply if we use the Morse function $F(\alpha)=\sqrt{E(\alpha)}$.

The free loop space $\Lambda$ consists of $H^{1}$ mappings $\alpha:[0,1] \rightarrow M$ such that $\alpha(0)=\alpha(1)$. It admits the structure of a Hilbert manifold $([\mathrm{K}]$, Cht $]$ ), so it is a complete metric space, hence paracompact and Hausdorff. The loop space $\Lambda$ is homotopy equivalent to the Frechet manifold of smooth loops $\beta: S^{1} \rightarrow M$. Denote by $\Lambda_{0}=\Lambda \leq 0 \cong M$ the space of constant loops. 
The energy of a loop depends on its parametrization; the length does not. Thus, $L(\alpha) \leq$ $F(\alpha)$ for all $\alpha \in \Lambda$, with equality if and only if the loop is parametrized proportionally to arc length (abbreviated PPAL), meaning that $\left|\alpha^{\prime}(t)\right|$ is constant. Every geodesic is, by definition, parametrized proportionally to arclength. A loop $\alpha \in \Lambda$ is a critical point of $F$ if and only if $\alpha$ is a closed geodesic. Let $\Sigma \subset \Lambda$ be the set of critical points of $F$, and set $\Sigma^{=a}=\Sigma \cap \Lambda^{=a}$.

The index and nullity of the critical points of $F$ coincide with those of $E$. Recall (for example, from [K] p. 57) that the index of a closed geodesic $\gamma$ is the dimension of a maximal subspace of $T_{\gamma}(\Lambda)$ on which the Hessian $d^{2} F(\gamma)$ is negative definite, and the nullity of $\gamma$ is $\operatorname{dim}\left(T_{\gamma}^{0} \Lambda\right)-1$ where $T_{\gamma}^{0} \Lambda$ is the null space of the Hessian $d^{2} F(\gamma)$. The -1 is incorporated to account for the fact that every closed geodesic $\gamma$ occurs in an $O(2)$ orbit of closed geodesics. The critical point $\gamma$ is nondegenerate if this single orbit is a nondegenerate Morse-Bott critical submanifold, or equivalently, if the nullity is zero. A number $a \in \mathbb{R}$ is a nondegenerate critical value if the critical set $\Sigma^{=a}$ consists of nondegenerate critical orbits. In this case there are finitely many critical orbits in $\Sigma^{=a}$ and the number $a \in \mathbb{R}$ is an isolated critical value. In $\$ 12$ we will encounter a critical set $\Sigma^{=a}$ of dimension $>1$ (consisting of geodesics with nullity $>0$ ), which is nondegenerate in the sense of Bott. In this case we say the critical value $a \in \mathbb{R}$ is nondegenerate in the sense of Bott. To distinguish "nondegenerate" from "nondegenerate in the sense of Bott", we will sometimes refer to the former case with the phrase "isolated nondegenerate critical orbit".

Denote by $\mathcal{A} \subset \Lambda$ the subspace of loops parametrized proportionally to arc length (PPAL). Then $F(\alpha)=L(\alpha)$ for all $\alpha \in \mathcal{A}$. We write $\mathcal{A}^{\leq a}$ (etc.) for those $\alpha \in \mathcal{A}$ such that $F(\alpha) \leq a$, cf. equation (1.1.2). The following result is due to Anosov $\mathrm{A}$.

2.2. Proposition. For all $a \leq \infty$ the inclusion $\mathcal{A}^{\leq a} \rightarrow \Lambda^{\leq a}$ is a homotopy equivalence. A homotopy inverse is the mapping $A: \Lambda \leq a \rightarrow \mathcal{A}^{\leq a}$ which associates to any path $\alpha$ the same path parametrized proportionally to arclength, with the same basepoint. It follows that the set of loops of length $\leq a$ also has the homotopy type of $\Lambda \leq a$.

2.3. The evaluation mapping $\mathbf{e v}_{s}: \Lambda \rightarrow M$ is given by $\mathbf{e v}_{s}(\alpha)=\alpha(s)$. The figure eight space $\mathcal{F}=\Lambda \times_{M} \Lambda$ is the pullback of the diagonal under the mapping

$$
\mathbf{e v}_{0} \times \mathbf{e v}_{0}: \Lambda \times \Lambda \rightarrow M \times M .
$$

It consists of composable pairs of loops. Denote by $\phi_{s}: \mathcal{F} \rightarrow \Lambda$ the mapping which joins the two loops at time $s$, that is,

$$
\phi_{s}(\alpha, \beta)(t)=\left\{\begin{array}{ll}
\alpha\left(\frac{t}{s}\right) & \text { for } t \leq s \\
\beta\left(\frac{t-s}{1-s}\right) & \text { for } s \leq t \leq 1
\end{array} .\right.
$$

The mapping $\phi_{s}$ is one to one. The energy of the composed loop $\phi_{s}(\alpha, \beta)$ is

$$
E\left(\phi_{s}(\alpha, \beta)\right)=\frac{E(\alpha)}{s}+\frac{E(\beta)}{1-s}
$$


which is minimized when

$$
s=\sqrt{E(\alpha)} /(\sqrt{E(\alpha)}+\sqrt{E(\beta)}) .
$$

2.4. Lemma. Consider $M=\Lambda_{0} \times_{M} \Lambda_{0}$ to be a subspace of $\mathcal{F}=\Lambda \times_{M} \Lambda$. Then the mapping $\phi_{\min }: \mathcal{F}-M \rightarrow \Lambda$ defined by $\phi_{\min }(\alpha, \beta)=\phi_{s}(\alpha, \beta)$ for

$$
s=\frac{F(\alpha)}{F(\alpha)+F(\beta)}
$$

extends continuously across $M$, giving a mapping $\phi_{\min }: \Lambda \times_{M} \Lambda \rightarrow \Lambda$ which is homotopic to the embedding $\phi_{s}: \mathcal{F} \rightarrow \Lambda$ for any $s \in(0,1)$, and which satisfies

$$
F\left(\phi_{\min }(\alpha, \beta)\right)=F(\alpha)+F(\beta) .
$$

If $\alpha$ and $\beta$ are PPAL then so is $\phi_{\min }(\alpha, \beta)$.

If $A, B \subset \Lambda$ write $A \times_{M} B=(A \times B) \cap\left(\Lambda \times_{M} \Lambda\right)$ and define $A * B=\phi_{\min }\left(A \times_{M} B\right)$ to be the subset consisting of all composed loops, glued together at the energy-minimizing time. Then $\Lambda^{\leq a} * \Lambda^{\leq b} \subset \Lambda^{\leq a+b}$.

2.5. By [Cht] Prop. 2.2.3 or [BO] Prop. 1.17, the figure eight space $\mathcal{F}=\Lambda \times_{M} \Lambda$ has an $n$ dimensional normal bundle $\nu$ and tubular neighborhood $N$ in $\Lambda \times \Lambda$ (see $§$ B.1) because the mapping (2.3.1) is a submersion whose domain is a Hilbert manifold. Similarly, for any $a, b \in \mathbb{R}$ the space

$$
\mathcal{F}^{<a,<b}=\left\{(\alpha, \beta) \in \Lambda^{<a} \times \Lambda^{<b}: \alpha(0)=\beta(0)\right\}
$$

has a normal bundle and tubular neighborhood in $\Lambda^{<a} \times \Lambda^{<b}$ and the image $\phi_{s}(\mathcal{F})$ has a normal bundle and tubular neighborhood in $\Lambda$ because it is the pre-image of the diagonal $\Delta \subset M \times M$ under the submersion

$$
\left(\mathbf{e v}_{0}, \mathbf{e v}_{s}\right): \Lambda \longrightarrow M \times M .
$$

The normal bundle of $\Delta$ in $M \times M$ is non-canonically isomorphic to the tangent bundle $T M$, so the normal bundle $\nu$ is orientable if $M$ is orientable.

\section{The finite dimensional approximation of Morse}

3.1. In this section we recall some standard facts concerning the finite dimensional approximation $\mathcal{M}$ to the free loop space $\Lambda$ of a smooth compact Riemannian manifold $M$. This finite dimensional approximation was described by Morse [Mo1] but his description is rather difficult to interpret by modern standards. It was clarified by Bott [Bo2] and further described by Milnor [Mi]. Related finite dimensional models are discussed in [BC].

Fix $\rho>0$ less than one half the injectivity radius of $M$. For points $x, y \in M$ which lie at a distance less than $\rho$, we will write $|x-y|$ for this distance. 
3.2. Lemma. Fix $N \geq 1$. Let $x=\left(x_{0}, x_{1}, \cdots, x_{N}\right) \in M^{N+1}$. Let $\alpha:[0,1] \rightarrow M$ be any piecewise smooth curve such that $\alpha(i / N)=x_{i}$. If $F(\alpha) \leq \rho \sqrt{N}$ then $\left|x_{i}-x_{i-1}\right| \leq \rho$ for each $i=1,2, \cdots, N$ and hence, for each $i$ there is a unique geodesic segment from $x_{i-1}$ to $x_{i}$. If $\gamma=\gamma(x)$ denotes the path obtained by patching these geodesic segments together, with $\gamma(i / N)=x_{i}$ then

$$
F(\gamma(x))=\sqrt{N \sum_{i=1}^{N}\left|x_{i}-x_{i-1}\right|^{2}} .
$$

Proof. Let $\alpha_{i}:\left[\frac{i-1}{N}, \frac{i}{N}\right] \rightarrow M$ denote the $i$-th segment of the path. Then $L\left(\alpha_{i}\right)^{2} \leq$ $E\left(\alpha_{i}\right) / N \leq \rho^{2}$. Therefore $\left|x_{i}-x_{i-1}\right| \leq \rho$. The energy of the resulting piecewise geodesic path $\gamma$ is therefore $E(\gamma)=\Sigma_{i=1}^{N} E\left(\gamma_{i}\right)=N \Sigma_{i=1}^{N}\left|x_{i}-x_{i-1}\right|^{2}$.

For $N \geq 1$ and $a \in \mathbb{R}$ let

$$
\mathcal{M}_{N}^{\leq a}=\left\{\left(x_{0}, x_{1}, \cdots, x_{N}\right) \in M^{N+1}: x_{0}=x_{N} \text { and } F(\gamma(x)) \leq a\right\} .
$$

According to Lemma 3.2 , if $a \leq \sqrt{N} \rho$ then we have a well defined mapping

$$
\gamma: \mathcal{M}_{N}^{\leq a} \hookrightarrow \Lambda
$$

3.3. Proposition. Suppose $a \leq \sqrt{N} \rho$. Then the mapping $F \circ \gamma: \mathcal{M}_{N}^{\leq a} \rightarrow \mathbb{R}$ is smooth and proper. The restrictions $\gamma: \mathcal{M}^{\leq a} \hookrightarrow \Lambda^{\leq a}$ and $\gamma: \mathcal{M}^{<a} \hookrightarrow \Lambda^{<a}$ are homotopy equivalences. The mapping $\gamma$ identifies the critical points (with values $\leq a$ ) of $F \circ \gamma$ with the critical points (with values $\leq a$ ) of $F$. The Morse index and nullity of each critical point are preserved under this identification. If, in addition, a is a regular value of $F$ or if a is a nondegenerate critical value of $F$ in the sense of Bott (cf. \$2.1) then the spaces $\mathcal{M}_{N}^{\leq a}$ and $\Lambda \leq a$ have the homotopy types of finite simplicial complexes.

Proof. There is a homotopy inverse $h: \Lambda^{\leq a} \rightarrow \mathcal{M}_{N}^{\leq a}$ which assigns to any loop $\alpha:[0,1] \rightarrow M$ the element $x=\left(x_{0}, \cdots, x_{N}\right)$ where $x_{i}=\alpha(i / N)$ for $0 \leq i \leq N$. Since $F(\alpha) \leq a$, Lemma 3.2 implies that $F \circ \gamma(h(\alpha)) \leq a$. The composition $h \circ \gamma$ is the identity. The composition $\gamma \circ h: \Lambda^{\leq a} \rightarrow \Lambda^{\leq a}$ is homotopic to the identity: we describe a homotopy $H_{T}$ from $\alpha \in \Lambda \leq a$ to $\gamma h(\alpha)$. Given $T \in[0,1]$ there exists $i$ such that $(i-1) / N \leq T \leq i / N$. The homotopy $H_{T}(\alpha)(t)$ coincides with $\alpha(t)$ for $t \leq(i-1) / N$. It coincides with the piecewise geodesic path $\gamma(\alpha)(t)$ for $t \geq(i / N)$. For $t$ in the interval $[(i-1) / N, i / N]$ the path $H_{T}(\alpha)(t)$ agrees with $\alpha$ for $t \leq T$ and it is geodesic on $[T, i / N]$. Replacing part of the curve $\alpha$ with a geodesic segment between the same two points does not increase its energy, so $H_{T}: \Lambda^{\leq a} \times[0,1] \rightarrow \Lambda \leq a$ is the desired homotopy.

If $a \in \mathbb{R}$ is a regular value then $\mathcal{M}_{N}^{\leq a}$ is a smooth compact manifold with boundary, so it can be triangulated, hence $\Lambda^{\leq a}$ is homotopy equivalent to a simplicial complex.

If the critical value $a$ of $F$ is nondegenerate in the sense of Bott, then $a$ is also a (Bott-) nondegenerate critical value of $F \circ \gamma$. It is then possible to Whitney stratify $\mathcal{M} \underset{N}{\leq a}$ so that $\mathcal{M}_{N}^{=a}$ is a closed union of strata. The complete argument is standard but technically messy; here is an outline. Each connected component of the singular set $S$ of $F \circ \gamma$ is a stratum. 
The set $\mathcal{M}_{N}^{=a}-S$ is another stratum; it is a manifold because it contains no critical points of $F \circ \gamma$. Finally, $\mathcal{M}_{N}^{<a}$ is the open stratum. According to the generalized Morse lemma, there exist local coordinate near each point $x$ in the critical set, with respect to which the function $F \circ \gamma$ has the form $F(\gamma(x))+\sum_{i=1}^{r} x_{i}^{2}-\Sigma_{i=r+1}^{s} x_{i}^{2}$ (with the last $n-s$ coordinates not appearing in the formula). Using this, it is possible to see that the above stratification satisfies the Whitney conditions.

Every Whitney stratified space can be triangulated ([Gor], [J]), so it follows that $\mathcal{M}_{N}^{\leq a}$ is homeomorphic to a finite simplicial complex, hence $\Lambda^{\leq a}$ is homotopy equivalent to a finite simplicial complex.

\section{Support, Critical values, and level homology}

4.1. Continue with the notation $M, \Lambda, F, \Sigma$ of $\oint 2$, Let $G$ be an Abelian group. A class $\alpha \in \check{H}_{i}(\Lambda ; G)$ is supported (in Čech homology, cf. Appendix $\mathrm{A}$ ) on a closed set $A \subset \Lambda$ if there is a class $\alpha^{\prime} \in \check{H}_{i}(A ; G)$ such that $\alpha=i_{*}\left(\alpha^{\prime}\right)$ where $i: A \rightarrow \Lambda$ is the inclusion. This implies that $\alpha \mapsto 0 \in \check{H}_{i}(\Lambda, A ; G)$ but the converse does not necessarily hold. Define the critical value $\operatorname{cr}(\alpha)$ to be the infemum

$$
\begin{aligned}
\operatorname{cr}(\alpha) & =\inf \left\{a \in \mathbb{R}: \alpha \in \operatorname{Image}\left(\check{H}_{i}\left(\Lambda^{\leq a} ; G\right) \rightarrow H_{i}(\Lambda ; G)\right)\right\} \\
& =\inf \left\{a \in \mathbb{R}: \alpha \text { is supported on } \Lambda^{\leq a}\right\} .
\end{aligned}
$$

A non-zero homology class $\alpha$ normally gives rise to a non-zero class $\beta$ in level homology at the level $\operatorname{cr}(\alpha)$. Let us say that two classes $\alpha \in H_{i}(\Lambda ; G)$ and $\beta \in \check{H}_{i}\left(\Lambda^{\leq a}, \Lambda^{<a} ; G\right)$ are associated if there exists an associating class $\omega \in \check{H}_{i}(\Lambda \leq a ; G)$ with

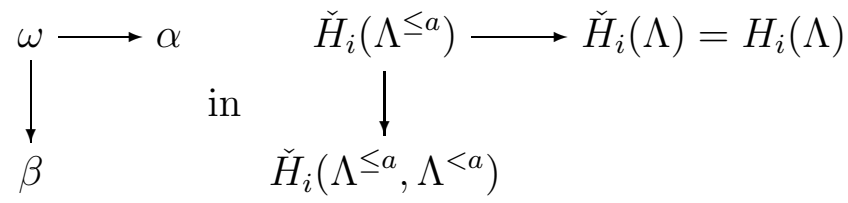

4.2. Lemma. Let $\alpha \in H_{i}(\Lambda ; G), \alpha \neq 0$. Then the following statements hold.

(1) $\operatorname{cr}(\alpha)$ is a critical value of $F$.

(2) $\operatorname{cr}(\alpha)$ is independent of the homology theory (Čech or singular) used in the definition.

(3) $\operatorname{cr}(\alpha)=\inf \left\{a \in \mathbb{R}: \alpha \in \operatorname{ker}\left(H_{i}(\Lambda) \rightarrow \check{H}_{i}(\Lambda, \Lambda \leq a ; G)\right)\right\}$.

If $a \in \mathbb{R}$ is a nondegenerate critical value in the sense of Bott, or if $G$ is a field, then

(4) $\operatorname{cr}(\alpha)=a$ if and only if there exists $0 \neq \beta \in H_{i}\left(\Lambda^{\leq a}, \Lambda^{<a} ; G\right)$ associated with $\alpha$.

(5) $\operatorname{cr}(\alpha)<a$ if and only if $\alpha$ is associated to the zero class $0=\beta \in H_{i}\left(\Lambda^{\leq a}, \Lambda^{<a} ; G\right)$.

Proof. For part (1), if $\operatorname{cr}(\alpha)$ were a regular value than the flow of $-\operatorname{grad}(F)$ would reduce the support of $\alpha$ below this value. Now let $b_{n} \downarrow \operatorname{cr}(\alpha)$ be a convergent sequence of regular values (which exists because the regular values of $F$ are dense in $\mathbb{R}$ ). By Lemma A.4 the Cech homology and singular homology of $\Lambda^{\leq b_{n}}$ coincide, which proves (2); and the homology 
sequence for the pair $\left(\Lambda, \Lambda^{\leq b_{n}}\right)$ is exact, which proves (3). If $a$ is a nondegenerate critical value (in the sense of Bott; cf. $\$ 2.1$ ) then the set $\Lambda^{\leq a}$ is a deformation retract of some open set $U \subset \Lambda$ which therefore contains $\Lambda^{\leq b_{n}}$ for sufficiently large $n$. Hence $\alpha$ is the image of some

$$
\omega \in H_{i}\left(\Lambda^{\leq b_{n}}\right) \rightarrow H_{i}(U) \cong H_{i}\left(\Lambda^{\leq a}\right) .
$$

Moreover the homology sequence for $\left(\Lambda^{\leq a}, \Lambda^{<a}\right)$ is exact, which proves (4) and (5). If $G$ is a field, see Lemma A.5.

4.3. Similarly, a cohomology class $\alpha \in H^{j}\left(\Lambda, \Lambda_{0} ; G\right)$ is supported on a closed set $B \subset$ $\Lambda-\Lambda_{0}$ if it maps to zero in $\breve{H}^{j}\left(\Lambda-B, \Lambda_{0} ; G\right)$ or equivalently, if it comes from a class in $\check{H}^{j}(\Lambda, \Lambda-B ; G)$. Define the critical value

$$
\begin{aligned}
\operatorname{cr}(\alpha) & =\sup \left\{b: \alpha \in \operatorname{ker}\left(H^{j}\left(\Lambda, \Lambda_{0} ; G\right) \rightarrow \check{H}^{j}\left(\Lambda^{<b}, \Lambda_{0} ; G\right)\right)\right\} \\
& =\sup \left\{b: \alpha \text { is supported on } \Lambda^{\geq b}\right\}
\end{aligned}
$$

Let us say the classes $\alpha \in H^{j}\left(\Lambda, \Lambda_{0} ; G\right)$ and $\beta \in \check{H}^{j}\left(\Lambda^{\leq b}, \Lambda^{<b} ; G\right)$ are associated if there exists $\omega \in \check{H}^{j}\left(\Lambda, \Lambda^{<b} ; G\right)$ with

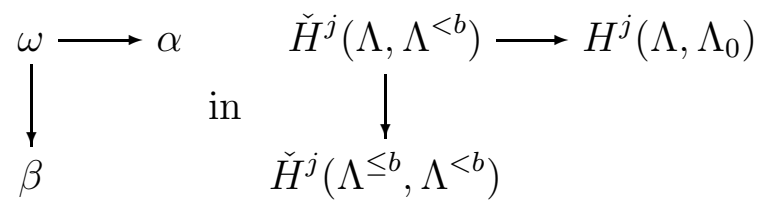

4.4. Lemma. Let $\alpha \in H^{j}\left(\Lambda, \Lambda_{0} ; G\right), \alpha \neq 0$. Then the following statements hold.

(1) $\operatorname{cr}(\alpha)$ is a critical value of $F$.

(2) $\operatorname{cr}(\alpha)$ is independent of the homology theory used in its definition.

If $a \in \mathbb{R}$ is a nondegenerate critical value in the sense of Bott, or if $G$ is a field then

(3) $\operatorname{cr}(\alpha)=b$ if and only if there exits $0 \neq \beta \in H^{j}\left(\Lambda^{\leq b}, \Lambda^{<b} ; G\right)$ associated with $\alpha$.

(4) $\operatorname{cr}(\alpha)<b$ if and only if $\alpha$ is associated with the zero class $0 \in H^{j}\left(\Lambda^{\leq b}, \Lambda^{<b} ; G\right)$.

Proof. The proof is the same as in Lemma 4.2.

\section{The Chas-Sullivan Product}

5.1. Throughout the next three sections $M$ denotes a connected Riemannian manifold of dimension $n$. We assume either (a) the manifold $M$ is orientable and oriented and coefficients for homology are taken in the ring of integers $G=\mathbb{Z}$ or (b) the manifold $M$ is not necessarily orientable, and coefficients for homology are taken in the ring $G=\mathbb{Z} /(2)$. We will often suppress mention of the coefficient ring $G$ when assumption (a) or (b) is in force. 
5.2. In [CS] a product $*: H_{i}(\Lambda) \times H_{j}(\Lambda) \rightarrow H_{i+j-n}(\Lambda)$ on the homology of the free loop space $\Lambda$ was defined. It has since been re-interpreted in a number of different contexts ([CKS], [CJ], [Co]). Recall, for example from [CJ] or [AS1] that it can be constructed as the following composition:

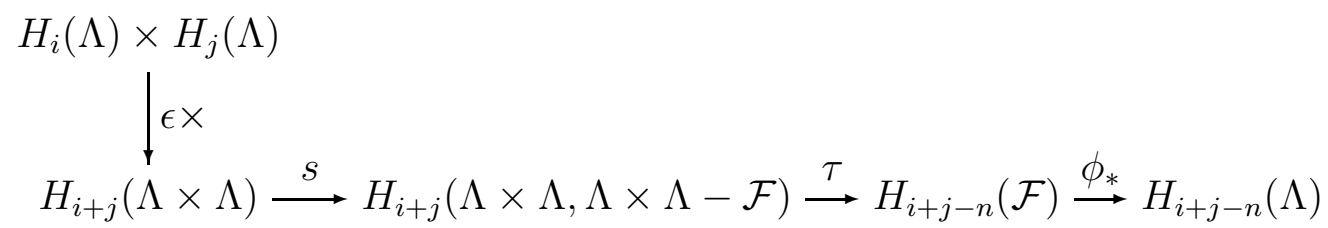

In this diagram, $\epsilon=(-1)^{n(n-j)}(\mathrm{cf}$. [D] VIII $\S 13.3$, Cht]), and $\times$ denotes the homology cross product. The map $\tau$ is the Thom isomorphism (B.1.2) for the normal bundle $\nu_{\mathcal{F}}$ of $\mathcal{F}=\Lambda \times_{M} \Lambda$ in $\Lambda \times \Lambda$ ( Cht Prop. 2.2.3, BO Prop. 1.17). The composition $\tau \circ s$ is a Gysin homomorphism (B.2.4). The map $\phi=\phi_{\frac{1}{2}}$ composes the two loops at time $t=1 / 2$. If $M$ is orientable then $\nu_{\mathcal{F}}$ is also orientable (\$2.4). We will often substitute the homotopic mapping $\phi_{\min }$ of Lemma 2.4 for $\phi_{\frac{1}{2}}$. The construction may be summarized as passing from the left to the right in the following diagram

$$
\Lambda \times \Lambda \longleftarrow \mathcal{F} \longrightarrow \Lambda
$$

It is well known that the Chas-Sullivan product is (graded) commutative, but this is not entirely obvious since it involves reversing the order of composition of loops, and the fundamental group of $M$ may be non-commutative. We include the short proof because the same method will be used in $\$ 8$.

5.3. Proposition. CS If $a \in H_{i}(\Lambda)$ and $b \in H_{j}(\Lambda)$ then $b * a=(-1)^{(i-n)(j-n)} a * b$.

Proof. The map $\sigma: \Lambda \times \Lambda \rightarrow \Lambda \times \Lambda$ that switches factors satisfies $\sigma_{*}(a \times b)=(-1)^{i j}(b \times a)$. It restricts to an involution $\sigma: \mathcal{F} \rightarrow \mathcal{F}$. Identifying $S^{1}=\mathbb{R} / \mathbb{Z}$, define $\chi_{r}: S^{1} \rightarrow S^{1}$ by $\chi_{r}(t)=t+r$. The (usual) action, $\widehat{\chi}: S_{1} \times \Lambda \rightarrow \Lambda$ of $S^{1}$ on $\Lambda$ is given by

$$
\widehat{\chi}_{r}(\gamma)=\gamma \circ \chi_{r}
$$

for $r \in S^{1}$. The action of $\widehat{\chi}_{\frac{1}{2}}$ preserves $\phi_{\frac{1}{2}}(\mathcal{F})$ and in fact

$$
\sigma(\gamma)=\widehat{\chi}_{\frac{1}{2}}(\gamma)=\beta \cdot \alpha
$$

for any $\gamma=\alpha \cdot \beta \in \phi_{\frac{1}{2}}(\mathcal{F})$ which is a composition of two loops $\alpha, \beta$ glued at time $1 / 2$. Let $\mu_{\mathcal{F}} \in H^{n}(\Lambda \times \Lambda, \Lambda \times \Lambda-\mathcal{F})$ be the Thom class of the normal bundle $\nu_{F}$. Then $\sigma^{*}\left(\mu_{\mathcal{F}}\right)=$ 
$(-1)^{n} \mu_{\mathcal{F}}$. Since $\chi_{r}: \Lambda \rightarrow \Lambda$ is homotopic to the identity we have:

$$
\begin{aligned}
(-1)^{n(n-i)} b * a & =\phi_{*}\left(\mu_{\mathcal{F}} \cap(b \times a)\right) \\
& =(-1)^{i j} \phi_{*}\left(\mu_{\mathcal{F}} \cap \sigma_{*}(a \times b)\right) \\
& =(-1)^{i j} \phi_{*} \sigma_{*}\left(\sigma^{*}\left(\mu_{\mathcal{F}} \cap(a \times b)\right)\right. \\
& =(-1)^{i j}(-1)^{n} \widehat{\chi}_{\frac{1}{2}} \phi_{*}\left(\mu_{\mathcal{F}} \cap(a \times b)\right) \\
& =(-1)^{i j}(-1)^{n}(-1)^{n(n-j)} a * b
\end{aligned}
$$

5.4. Proposition. If $M$ is oriented let $G=\mathbb{Z}$ otherwise let $G=\mathbb{Z} /(2)$. Let $\alpha, \beta \in H_{*}(\Lambda ; G)$ be homology classes supported on closed sets $E, F \subset \Lambda$ respectively. Then $\alpha * \beta$ is supported on the closed set $E * F=\phi_{\min }\left(E \times_{M} F\right)$. In particular,

$$
\operatorname{cr}(\alpha * \beta) \leq \operatorname{cr}(\alpha)+\operatorname{cr}(\beta) \text {. }
$$

For any $a, b$ with $0 \leq a, b \leq \infty$ the Chas-Sullivan product extends to a family of products,

$$
\check{H}_{i}\left(\Lambda^{\leq a} ; G\right) \times \check{H}_{j}\left(\Lambda^{\leq b} ; G\right) \rightarrow \check{H}_{i+j-n}\left(\Lambda^{\leq a+b} ; G\right)
$$

and for any $0 \leq a^{\prime}<a \leq \infty$ and $0 \leq b^{\prime}<b \leq \infty$ to products

$$
\begin{aligned}
& \check{H}_{i}\left(\Lambda^{\leq a}, \Lambda^{\leq a^{\prime}} ; G\right) \times \check{H}_{j}\left(\Lambda^{\leq b}, \Lambda^{\leq b^{\prime}} ; G\right) \rightarrow \check{H}_{i+j-n}\left(\Lambda^{\leq a+b}, \Lambda^{\leq \max \left(a+b^{\prime}, a^{\prime}+b\right)} ; G\right) \\
& \check{H}_{i}\left(\Lambda^{\leq a}, \Lambda^{<a} ; G\right) \times \check{H}_{j}\left(\Lambda^{\leq b}, \Lambda^{<b} ; G\right) \rightarrow \check{H}_{i+j-n}\left(\Lambda^{\leq a+b}, \Lambda^{<a+b} ; G\right) .
\end{aligned}
$$

These products are compatible under the mappings induced by inclusion. If the set $\mathrm{cr} \subset \mathbb{R}$ of critical values is discrete then we obtain a ring structure on the level homology

$$
\underset{a \in \mathrm{cr}}{\oplus} \check{H}_{*}\left(\Lambda^{\leq a}, \Lambda^{<a} ; R\right)
$$

Proof. First we construct, for any open sets $A^{\prime} \subset A \subset \Lambda$ and $B^{\prime} \subset B \subset \Lambda$ a product

$$
H_{i}\left(A, A^{\prime} ; G\right) \times H_{j}\left(B, B^{\prime} ; G\right) \rightarrow H_{i+j-n}\left(A * B, A * B^{\prime} \cup A^{\prime} * B\right)
$$

on singular homology, as shown in Figure 5.2.

In this diagram, $\times$ denotes the homology cross product, $\epsilon=(-1)^{n(n-j)}$, and the Thom isomorphism $\tau$ of Proposition B.2 is applied to the triple

$$
\left(A-A^{\prime}\right) \times_{M}\left(B-B^{\prime}\right) \subset A \times_{M} B \subset A \times B .
$$

The hypotheses of Proposition B.2 are satisfied because $A \times B$ is a Hilbert manifold, so $A \times{ }_{M} B$ has a normal bundle and tubular neighborhood in $A \times B$, and because the subspace $\left(A-A^{\prime}\right) \times_{M}\left(B-B^{\prime}\right)$ is closed in $A \times_{M} B$. 


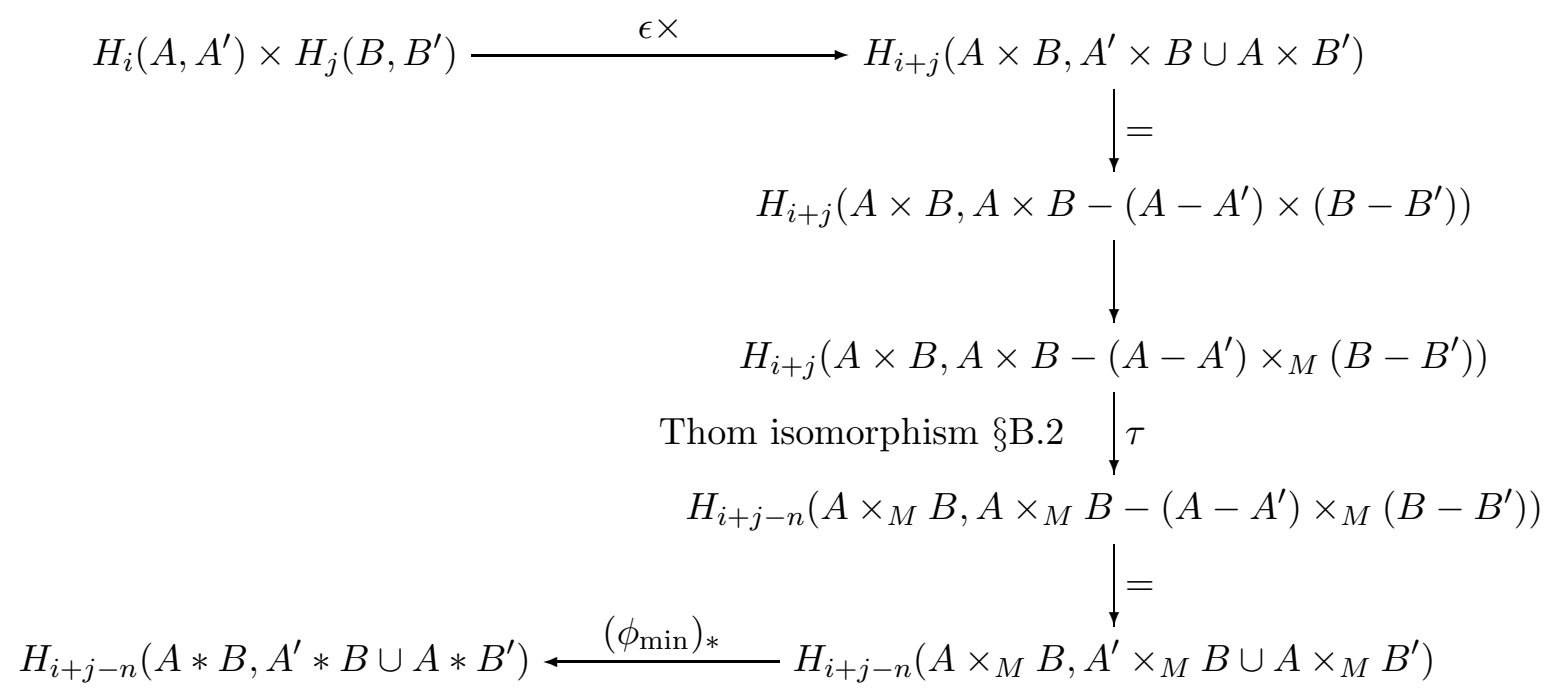

Figure 1. Relative Chas-Sullivan Product

It is easy to see that this product is compatible with the product in $\$ 5.2$ in the sense that the following diagram commutes:

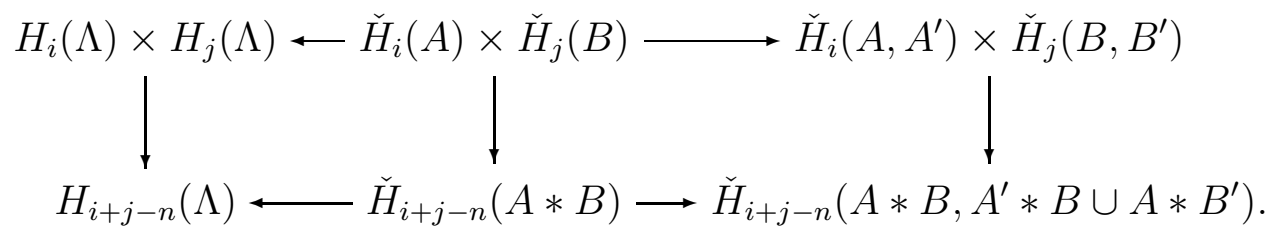

Now the other products may be obtained by a limiting procedure using Lemma A.5. The statement about the support of $\alpha * \beta$ follows by taking a sequence of open neighborhoods $A_{n} \downarrow E$ and $B_{n} \downarrow F$. To construct the product (5.4.3) for example, start with

$$
H_{i}\left(\Lambda^{<a+\epsilon}, \Lambda^{<a^{\prime}-\delta}\right) \times H_{j}\left(\Lambda^{<b+\epsilon}, \Lambda^{<b^{\prime}-\delta}\right) \rightarrow H_{i+j-n}\left(\Lambda^{<a+b+\epsilon-\delta}, \Lambda^{<\max \left(a^{\prime}+b+\epsilon-\delta, a+b^{\prime}+\epsilon-\delta\right)}\right)
$$

where $\delta>\epsilon$. Taking the (inverse) limit as $\epsilon \downarrow 0$ gives a pairing

$$
\check{H}_{i}\left(\Lambda^{\leq a}, \Lambda^{<a-\delta}\right) \times \check{H}_{j}\left(\Lambda^{\leq b}, \Lambda^{<b-\delta}\right) \rightarrow \check{H}_{i+j-n}\left(\Lambda^{\leq a+b}, \Lambda^{<\max \left(a^{\prime}+b-\delta, a+b^{\prime}-\delta\right)}\right) .
$$

Taking the direct limit as $\delta \downarrow 0$ (and recalling from $\$$ A.2 that homology commutes with direct limits) gives the pairing (5.4.3). The other products are similarly constructed. (The Chas-Sullivan product can even be constructed this way, cf. [Cht].) This completes the proof of Proposition 5.4.

5.5. If $\alpha=[A, \partial A]$ and $\beta=[B, \partial B]$ are the fundamental classes of manifolds $(A, \partial A) \subset$ $\left(\Lambda^{\leq a}, \Lambda^{\leq a^{\prime}}\right)$ and $(B, \partial B) \subset\left(\Lambda^{\leq b}, \Lambda^{\leq b^{\prime}}\right)$ which are transverse over $M$, then the C-S product $[\alpha] *[\beta]$ is represented by the fundamental class of the manifold

$$
\phi_{\min }\left(A \times_{M} B, A \times_{M} \partial B \cup \partial A \times_{M} B\right)=(A * B, \partial(A * B)) .
$$


For equations (11.5.2) and (12.5.2) we will need a similar fact about homology classes $\alpha, \beta$ which are supported on $(A, \partial A)$ and $(B, \partial B)$ but which are not necessarily the fundamental classes. The following proposition is more or less the original definition of the product $*$ from [CS]:

5.6. Proposition. Let $(A, \partial A)$ and $(B, \partial B)$ be smooth manifolds with boundary. Let $(A, \partial A) \rightarrow$ $\left(\Lambda^{<\alpha}, \Lambda^{<\alpha^{\prime}}\right)$ and $(B, \partial B) \rightarrow\left(\Lambda^{<\beta}, \Lambda^{<\beta^{\prime}}\right)$ be smooth embeddings, where $\alpha^{\prime}<\alpha$ and $\beta^{\prime}<\beta$. Assume the mappings $\mathbf{e v}_{0}: A \rightarrow M$ and $\mathbf{e v}_{0}: B \rightarrow M$ are transverse. If $M$ is oriented, then assume $A, B$ are orientable and oriented. Let $\alpha \in H_{i}(A, \partial A)$ and $\beta \in H_{i}(B, \partial B)$. Denote their images in the homology of $\Lambda$ by $[\alpha] \in H_{i}\left(\Lambda^{<a}, \Lambda^{<a^{\prime}}\right)$ and $[\beta] \in H_{j}\left(\Lambda^{<b}, \Lambda^{<b^{\prime}}\right)$. Define

$$
\alpha * \beta \in H_{i+j-n}\left(A \times_{M} B, A \times_{M} \partial B \cup \partial A \times_{M} B\right) \cong H_{i+j-n}\left(A \times_{M} B, A \times_{M} B-A^{\prime} \times_{M} B^{\prime}\right)
$$

to be the image of $(\alpha, \beta)$ under the following composition.

$$
\begin{gathered}
H_{i}(A, \partial A) \times H_{j}(B, \partial B) \longrightarrow H_{i+j}\left(A \times B, A \times B-A^{\prime} \times B^{\prime}\right) \\
H_{i+j-n}\left(A \times_{M} B, A \times_{M} B-A^{\prime} \times_{M} B^{\prime}\right) \frac{\tau}{\left(\frac{\tau .2)}{(B .2)}\right.} H_{i+j}\left(A \times B, A \times B-A^{\prime} \times_{M} B^{\prime}\right)
\end{gathered}
$$

Then $[\alpha] *[\beta]=\left(\phi_{\min }\right)_{*}(\alpha * \beta) \in H_{i+j-n}\left(\Lambda^{<a+b}, \Lambda^{\max \left(a+b^{\prime}, a^{\prime}+b\right)}\right)$.

Proof. The transversality assumption is equivalent to the statement that the mapping

$$
\left(\mathbf{e v}_{0}, \mathbf{e v}_{0}\right): A \times B \rightarrow M \times M
$$

is transverse to the diagonal $\Delta$. By [Cht Prop. 2.2.3 or [BO] Prop. 1.17, the intersection $A \times{ }_{M} B=A \times B \cap \mathcal{F}$ has a tubular neighborhood in $A \times B$ (with normal bundle $\nu_{\mathcal{F}} \mid\left(A \times_{M} B\right)$. As in the proof of Proposition 5.4 this makes it possible to apply the Thom isomorphism (B.2). Then the diagram (5.6.1) maps, term by term, to the diagram in the proof of Proposition 5.4 where the relative C-S product is defined. The Proposition amounts to the statement that these mappings commute, which they obviously do.

\section{Index growth}

Continue with the notation $M, \Lambda, F, \Sigma$ of $₫ 2$.

6.1. Proposition. Let $\gamma$ be a closed geodesic with index $\lambda$ and nullity $\nu$. Let $\lambda_{m}$ and $\nu_{m}$ denote the index and nullity of the $m$-fold iterate $\gamma^{m}$. Then $\nu_{m} \leq 2(n-1)$ for all $m$ and

$$
\begin{aligned}
\left|\lambda_{m}-m \lambda\right| & \leq(m-1)(n-1) \\
\left|\lambda_{m}+\nu_{m}-m(\lambda+\nu)\right| & \leq(m-1)(n-1) .
\end{aligned}
$$


The average index

$$
\lambda_{a v}=\lim _{m \rightarrow \infty} \frac{\lambda_{m}}{m}
$$

exists and

$$
\left|\lambda-\lambda_{a v}\right| \leq n-1 \text { and }\left|\lambda+\nu-\lambda_{a v}\right| \leq n-1 .
$$

Now assume $\gamma$ and $\gamma^{2}$ are nondegenerate critical points, i.e. they lie on isolated nondegenerate critical orbits. Then the inequalities in (6.1.3) are strict, and if $m$ is sufficiently large then the inequality (6.1.1) is also strict. Moreover if we let

$$
\begin{aligned}
& \lambda_{m}^{\min }=m \lambda_{1}-(m-1)(n-1) ; \\
& \lambda_{m}^{\max }=m \lambda_{1}+(m-1)(n-1)
\end{aligned}
$$

be the greatest and smallest possible values for $\lambda_{m}$ that are compatible with (6.1.1) then

$$
\begin{aligned}
& \lambda_{m}>\lambda_{m}^{\min } \Longrightarrow \lambda_{j}>\lambda_{j}^{\min } \text { for all } j>m ; \\
& \lambda_{m}<\lambda_{m}^{\max } \Longrightarrow \lambda_{j}<\lambda_{j}^{\max } \quad \text { for all } j>m .
\end{aligned}
$$

Much of this is standard and is well-known to experts, (see the references at the beginning of Appendix (C) but for completeness we include a proof based on the following well-known facts:

6.2. Well-Known Facts. Let $M$ be an $n$ dimensional Riemannian manifold. Let $\gamma$ be a closed geodesic. The Poincare map $P$ (linearization of the geodesic flow at a periodic point) is in $S p(2(n-1), \mathbb{R})$ and is defined up to conjugation. The index formula of Bott is

$$
\lambda_{m}=\operatorname{index}\left(\gamma^{m}\right)=\sum_{\omega^{m}=1} \Omega_{\gamma}(\omega) .
$$

where the $\omega$-index $\Omega_{\gamma}$ is an integer valued function defined on the unit circle with $\Omega_{\gamma}(\omega)=$ $\boldsymbol{\Omega}_{\gamma}(\bar{\omega})$. The function $\boldsymbol{\Omega}_{\gamma}$ is constant except at the eigenvalues of $P$. Its jump at each eigenvalue is determined by the splitting numbers $S_{P}^{ \pm}(\omega) \epsilon \mathbb{Z}$ :

$$
\lim _{\varepsilon \rightarrow 0^{+}} \Omega_{\gamma}\left(\omega e^{ \pm i \varepsilon}\right)=\Omega_{\gamma}(\omega)+S_{P}^{ \pm}(\omega)
$$

which depend only upon the conjugacy class of $P$. The nullity satisfies

$$
\nu_{m}=\operatorname{nullity}\left(\gamma^{m}\right)=\sum_{\omega^{m}=1} \mathcal{N}_{P}(\omega)
$$

where $\mathcal{N}_{P}(\omega):=\operatorname{dim} \operatorname{ker}(P-\omega I)$. The numbers $S_{P}^{ \pm}(\omega)$ and $\mathcal{N}_{P}(\omega)$ are additive on indecomposable symplectic blocks, and on each block

$$
\begin{aligned}
& S_{P}^{ \pm}(\omega) \epsilon\{0,1\} \\
& \mathcal{N}_{P}(\omega)-S_{P}^{ \pm}(\omega) \epsilon\{0,1\}
\end{aligned}
$$


6.3. Proof of Proposition 6.1. Assuming the above facts (6.2.16.2.5), the index-plusnullity satisfies

$$
\begin{aligned}
\lambda_{m}+\nu_{m}=\text { index-plus-nullity }\left(\gamma^{m}\right) & =\sum_{\omega^{m}=1} \Upsilon_{\gamma}(\omega) \\
\lim _{\varepsilon \rightarrow 0^{+}} \Upsilon_{\gamma}\left(\omega e^{ \pm i \varepsilon}\right) & =\Upsilon_{\gamma}(\omega)-T_{P}^{ \pm}(\omega) ;
\end{aligned}
$$

where

$$
\begin{aligned}
& \Upsilon_{\gamma}(\omega):=\Omega_{\gamma}(\omega)+\mathcal{N}_{P}(\omega) ; \\
& T_{P}^{ \pm}(\omega):=\mathcal{N}_{P}(\omega)-S_{P}^{ \pm}(\omega) .
\end{aligned}
$$

Thus $T_{P}^{ \pm}(\omega)$ is additive and takes values in $\{0,1\}$ on each indecomposable block, and $-\left(\lambda_{m}+\right.$ $\left.\nu_{m}\right)$ and $-\Upsilon_{\gamma}$ have the same formal properties (6.2.1, 6.2.4) as $\lambda_{m}$ and $\boldsymbol{\Omega}_{\gamma}$. Thus a proof of the statements about $\lambda_{m}$ using only these three properties will also serve as a proof of the statements about $\lambda_{m}+\nu_{m}$.

As a consequence of equations (6.2.1,6.2.4) we have:

$$
\left|\boldsymbol{\Omega}_{\gamma}(\omega)-\boldsymbol{\Omega}_{\gamma}(\tau)\right| \leq n-1 \text { for all } \omega, \tau
$$

Moreover if $\left|\Omega_{\gamma}(\omega)-\Omega_{\gamma}(\tau)\right|=n-1$, with $\operatorname{Re} \omega<\operatorname{Re} \tau$, then all the eigenvalues of $P$ lie in the unit circle, with real part in $[\operatorname{Re} \omega, \operatorname{Re} \tau]$. (To see this, note that each indecomposable block has dimension at least 2.) Equation (6.1.1) and the first half of (6.1.3) follow. Moreover,

$$
\lambda_{a v}=\frac{1}{2 \pi} \int_{0}^{2 \pi} \Omega_{\gamma}\left(e^{i t}\right) d t
$$

Equality in 6.1.1 implies that $\lambda_{m}=\lambda_{m}^{\max }$ or $\lambda_{m}=\lambda_{m}^{\min }$. Equality in the first half of 6.1.3, together with (6.3.3) and (6.3.4) implies that $\left|\Omega_{\gamma}(\omega)-\Omega_{\gamma}(1)\right|=n-1$ almost everywhere on the circle, that 1 is the only eigenvalue of $P$, and thus that $\lambda_{m}=\lambda_{m}^{\max }$ for all $m$ or $\lambda_{m}=\lambda_{m}^{\min }$ for all $m$.

If $\gamma$ and $\gamma^{2}$ are nondegenerate, then neither -1 nor 1 is an eigenvalue of $P$. Suppose that $\lambda_{2}=\lambda_{2}^{\max }=2 \lambda_{1}+n-1$. Then (using the Bott formula) $\Omega_{\gamma}(0)=\lambda_{1}$ and $\boldsymbol{\Omega}_{\gamma}(-1)=$ $\lambda_{1}+n-1$. It follows that all the eigenvalues of $P$ lie on the unit circle. Furthermore

$$
\lambda_{k}=\lambda_{k}^{\max }=\lambda_{1}+(k-1)(n-1)
$$

if and only if all the $k^{\text {th }}$ roots of unity except for 1 lie to the left of all the eigenvalues of $P$ on the unit circle. The statements about strict inequality and equations (6.1.4), (6.1.5) follow. This concludes the proof of Proposition 6.1.

The following lemma will be used in the proof of Theorem 11.3.

6.4. Lemma. Fix a basepoint $x_{0} \in M$ and let $\Omega=\Omega_{x_{0}}=\mathbf{e v}_{0}^{-1}\left(x_{0}\right)$ be the Hilbert manifold of loops that are based at $x_{0}$. Let $\gamma \in \Omega$ be a closed geodesic, all of whose iterates are nondegenerate. Let $\lambda_{r}$ be the Morse index of the iterate $\gamma^{r}$. 
(i) Suppose the index growth is maximal for $r=n$, that is, $\lambda_{n}=n \lambda_{1}+(n-1)^{2}$. Then the index $\lambda_{1}^{\Omega}$ for $\gamma$ in the based loop space is equal to the index $\lambda_{1}$ for $\gamma$ in the free loop space.

(ii) Suppose the index growth is minimal for $r=n$, that is, $\lambda_{n}=n \lambda_{1}-(n-1)^{2}$. Then the difference between $\lambda_{1}$ and $\lambda_{1}^{\Omega}$ is maximal, that is, $\lambda_{1}=\lambda_{1}^{\Omega}+n-1$.

Proof. Let $T_{\gamma(0)}^{\perp} M$ be the subspace that is orthogonal to the tangent vector $\gamma^{\prime}(0)$. Let $T_{\gamma}^{\perp} \Lambda$ (resp. $T_{\gamma}^{\perp} \Omega$ ) be the subspace of vector fields $V(t)$ along $\gamma$ with $V(t) \perp \gamma^{\prime}(t)$ for all $t$. By a standard argument, for all $r \geq 1, \lambda_{r}$ (resp. $\lambda_{r}^{\Omega}$ ) is the dimension of a maximal negative subspace of $T_{\gamma^{r}}^{\perp} \Lambda$ (resp. of $T_{\gamma^{r}}^{\perp}(\Omega)$ ). Let $W_{r} \subset T_{\gamma^{r}}^{\perp} \Lambda$ be a maximal negative subspace. Let $K_{r}$ be the kernel of the map

$$
\begin{gathered}
W_{r} \stackrel{\phi}{\longrightarrow} T_{\gamma(0)}^{\perp} M \times T_{\gamma(0)}^{\perp} M \times \cdots \times T_{\gamma(0)}^{\perp} M \\
V \quad \mapsto\left(V(0), V\left(\frac{1}{r}\right), \cdots, V\left(\frac{r-1}{r}\right)\right) .
\end{gathered}
$$

The dimension of the image of this map is $\leq r(n-1)$ so $\operatorname{dim}\left(K_{r}\right) \geq \lambda_{r}-r(n-1)$. On the other hand, the kernel of $\phi$ on $T_{\gamma^{r}}^{\perp} \Lambda$ is the direct sum of $r$ copies of $T_{\gamma}^{\perp} \Omega$ in a way that is compatible with the Hessian of $F$, so the index of $F$ on this kernel is $r \lambda_{1}^{\Omega}$. Thus, for all $r \geq 1$ we have,

$$
r \lambda_{1}^{\Omega} \geq \operatorname{dim}\left(K_{r}\right) \geq \lambda_{r}-r(n-1) .
$$

Now consider Part (i). Clearly $\lambda_{1}^{\Omega} \leq \lambda_{1}$ so we need to verify the opposite inequality. But taking $r=n$ and $\lambda_{n}=n \lambda_{1}+(n-1)^{2}$ in (6.4.1) gives $\lambda_{1}^{\Omega} \geq \lambda_{1}-\frac{n-1}{n}$ as claimed.

Now consider Part (ii). Taking $r=1$ in (6.4.1) gives $\lambda_{1}^{\Omega} \geq \lambda_{1}-(n-1)$. However it is also clear that $\lambda_{n} \geq \lambda_{n}^{\Omega} \geq n \lambda_{1}^{\Omega}$ (because we can concatenate $n$ negative vector fields along $\gamma$ to obtain a negative vector field along $\left.\gamma^{n}\right)$. Taking $\lambda_{n}=n \lambda_{1}-(n-1)^{2}$ gives

$$
\lambda_{1}-\frac{(n-1)^{2}}{n}=\lambda_{1}-(n-1)+\frac{n-1}{n} \geq \lambda_{1}^{\Omega} .
$$

\section{Level nilpotence}

7.1. Let $R$ be a commutative ring with unit, let $a \in \mathbb{R}$, and let $\beta \in \check{H}_{i}\left(\Lambda^{\leq a}, \Lambda^{<a} ; R\right)$. We way that $\beta$ is level nilpotent if there exists $m$ such that the Chas-Sullivan product in level homology vanishes:

$$
0=\beta^{* m}=\beta * \beta * \cdots * \beta \in \check{H}_{m i+(m-1) n}\left(\Lambda^{\leq m a}, \Lambda^{<m a} ; R\right) .
$$

Let $\alpha \in \check{H}_{i}(\Lambda ; R)$. We say that $\alpha$ is level nilpotent if there exists $m$ such that $\operatorname{cr}\left(\alpha^{* m}\right)<$ $\operatorname{mcr}(\alpha)$.

7.2. Lemma. Let $\alpha \in \check{H}_{i}(\Lambda ; R), \alpha \neq 0$, and let $a=\operatorname{cr}(\alpha)$. Let $\beta \in \check{H}_{i}\left(\Lambda^{\leq a}, \Lambda^{<a} ; R\right)$ be an associated class. (A non-zero such class $\beta$ exists when a is a nondegenerate critical value in the sense of Bott, cf. Lemma 4.2.) If $\beta$ is level nilpotent then $\alpha$ is also level nilpotent. 
Proof. Let $\omega \in H_{i}\left(\Lambda^{\leq a} ; R\right)$ be a class which associates $\alpha$ and $\beta$. Then $\omega^{* m} \in \check{H}_{b}\left(\Lambda^{\leq m a} ; R\right)$ associates $\alpha^{* m}$ and $\beta^{* m}$, where $b=m i+(m-1) n$. But $\beta^{* m}=0$ if $m$ is sufficiently large, which implies by Lemma 4.2 that $\operatorname{cr}\left(\alpha^{* m}\right)<m a$.

7.3. Theorem. Let $F: \Lambda \rightarrow \mathbb{R}$ as above. If $M$ is orientable let $G=\mathbb{Z}$, otherwise let $G=\mathbb{Z} /(2)$. Suppose all the critical orbits of $F$ are isolated and nondegenerate. Then every homology class $\alpha \in H_{i}(\Lambda ; G)$ is level nilpotent, and for every a $\in \mathbb{R}$, every level homology class $\beta \in H_{i}\left(\Lambda^{\leq a}, \Lambda^{<a} ; G\right)$ is level nilpotent.

Proof. By Lemma 7.2 it suffices to prove that $\beta \in H_{i}\left(\Lambda^{\leq a}, \Lambda^{<a} ; G\right)$ is level nilpotent, where $a \in \mathbb{R}$ is a nondegenerate critical value. We suppress mention of the coefficient ring $G$. The critical set $\Sigma^{=a}=\Sigma(F) \cap F^{-1}(a)$ consists of the $S^{1}$ orbits of finitely many closed geodesics, say, $\gamma_{1}, \cdots, \gamma_{r}$. Let $\bar{\gamma}$ denote the $S^{1}$ orbit of $\gamma$. For $1 \leq j \leq r$ let $U_{j} \subset \Lambda$ be a neighborhood of $\bar{\gamma}_{j}$, chosen so that $U_{j} \cap U_{k}=\phi$ whenever $j \neq k$. We may choose the ordering so that there exists $s \leq r$ such that $H_{i}\left(\Lambda^{\leq a} \cap U_{j}, \Lambda^{<a} \cap U_{j}\right) \neq 0$ if and only if $1 \leq j \leq s$. The $\gamma_{j}$ with $1 \leq j \leq s$ are the critical points that are relevant to $\beta$, and Theorem C.2 implies that the index of $\gamma_{j}(1 \leq j \leq s)$ is either $i$ or $i-1$.

Set $\Sigma_{0}^{=a}=\cup_{j=1}^{s} \bar{\gamma}_{j}$. Using Proposition A.6, the level homology group is a direct sum

$$
H_{i}\left(\Lambda^{\leq a}, \Lambda^{<a}\right) \cong \oplus_{j=1}^{r} H_{i}\left(\Lambda^{\leq a} \cap U_{j}, \Lambda^{<a} \cap U_{j}\right)
$$

Since these factors vanish for $j>s$ we have canonical isomorphisms

$$
\begin{aligned}
H_{i}\left(\Lambda^{\leq a}, \Lambda^{<a}\right) & \cong \oplus_{j=1}^{s} H_{i}\left(U_{j}^{<a} \cup \bar{\gamma}_{j}, U_{j}^{<a}\right) \\
& \cong H_{i}\left(\Lambda^{<a} \cup \Sigma_{0}^{=a}, \Lambda^{<a}\right)
\end{aligned}
$$

using excision and homotopy equivalences. By comparing the long exact sequence for the pair $\left(\Lambda^{\leq a}, \Lambda^{<a}\right)$ with the long exact sequence for the pair $\left(\Lambda^{<a} \cup \Sigma_{0}^{=a}, \Lambda^{<a}\right)$ and using the five lemma, we conclude that the inclusion induces an isomorphism

$$
H_{i}\left(\Lambda^{<a} \cup \Sigma_{0}^{=a}\right) \cong H_{i}\left(\Lambda^{\leq a}\right) .
$$

Therefore $\beta$ is supported on $\Lambda^{<a} \cup \Sigma_{0}^{=a}$ so for any $m \geq 1, \beta^{* m}$ is supported on

$$
\left(\Lambda^{<a} \cup \Sigma_{0}^{=a}\right)^{* m} \subset \Lambda^{<m a} \cup\left(\Sigma_{0}^{=a}\right)^{* m} .
$$

The only critical points in $\left(\Sigma_{0}^{=a}\right)^{* m}$ are the $m$-fold iterates of the geodesics in $\bar{\gamma}_{j}$ (with $1 \leq j \leq s)$. Thus, using an arbitrarily brief flow along the trajectories of $-\nabla F$ we obtain an isomorphism

$$
H_{b}\left(\Lambda^{<m a} \cup\left(\Sigma_{0}^{=a}\right)^{* m}, \Lambda^{<m a}\right) \cong \stackrel{s}{\oplus} H_{b=1}\left(U_{j, m}^{<m a} \cup \overline{\gamma_{j}^{m}}, U_{j, m}^{<m a}\right) \ni \beta^{* m}
$$

where the $U_{j, m}$ are disjoint neighborhoods of the $\overline{\gamma_{j}^{m}}$ containing no other critical points, and where

$$
b=m i-(m-1)(n-1) .
$$


(There may be other critical points at level $m a$, but the support of $\beta^{* m}$ does not contain such points.)

We will now show that each of the summands on the right hand side of equation (7.3.1) vanishes if $m$ is sufficiently large. Fix $j$ with $1 \leq j \leq s$ and let $\lambda_{m}$ denote the index of $\gamma_{j}^{m}$. According to Theorem C.2. $\lambda_{1} \in\{i-1, i\}$. If the $j$-th summand in (17.3.1) is not zero then $\lambda_{m} \in\{b-1, b\}$. But this contradicts the strict inequality in (6.1.1), which holds if $m$ is sufficiently large.

\section{Cohomology products}

8.1. Throughout this section we take cohomology with coefficients in the integers $\mathbb{Z}$ if $M$ is orientable (in which case we assume an orientation has been chosen); otherwise in $\mathbb{Z} /(2)$. To simplify the notation, we suppress further mention of these coefficients. It is possible to define a product in cohomology, exactly along the same lines as the Chas-Sullivan product in $\$ 5.2$, as the composition

$$
H^{i}(\Lambda) \times H^{j}(\Lambda) \rightarrow H^{i+j}(\Lambda \times \Lambda) \rightarrow H^{i+j}(\mathcal{F}) \stackrel{\cong}{\longrightarrow} H^{i+j+n}\left(\Lambda, \Lambda-\phi_{\frac{1}{2}}(\mathcal{F})\right) \rightarrow H^{i+j+n}(\Lambda)
$$

(with $\phi_{\frac{1}{2}}: \mathcal{F} \rightarrow \Lambda$ as in 92.3 ) using the Thom isomorphism for the normal bundle of the figure eight space $\phi_{\frac{1}{2}}(\mathcal{F}) \subset \Lambda$. However we will show below that this product is zero when $i, j>n$. Instead, we will construct a cohomology product,

$$
H^{i}\left(\Lambda, \Lambda_{0}\right) \times H^{j}\left(\Lambda, \Lambda_{0}\right) \stackrel{\circledast}{\longrightarrow} H^{i+j+n-1}\left(\Lambda, \Lambda_{0}\right)
$$

using the following mapping

$$
J: \Lambda \times[0,1] \rightarrow \Lambda \text { given by } J(\alpha, s)=\alpha \circ \theta_{\frac{1}{2} \rightarrow s} .
$$

Here, $\theta=\theta_{\frac{1}{2} \rightarrow s}:[0,1] \rightarrow[0,1]$ is the reparametrization function that is linear on $\left[0, \frac{1}{2}\right]$, linear on $\left[\frac{1}{2}, 1\right]$, has $\theta(0)=0, \theta(1)=1$, and $\theta\left(\frac{1}{2}\right)=s$, see Figure 8.1 and 88.9

Let $\mathcal{F}^{>0,>0}=\left(\Lambda-\Lambda_{0}\right) \times_{M}\left(\Lambda-\Lambda_{0}\right)$ denote the set of composable pairs $(\alpha, \beta)$ such that $F(\alpha)>0$ and $F(\beta)>0$. The relative Thom isomorphism (B.2.1) for cohomology gives

$$
H^{m}\left(\mathcal{F}, \mathcal{F}-\mathcal{F}^{>0,>0}\right) \cong H^{m+n}\left(\Lambda, \Lambda-\phi_{\frac{1}{2}}\left(\mathcal{F}^{>0,>0}\right) .\right.
$$

The cohomology product is then the composition down the left hand column of Figure 3 , Here, $\omega=(-1)^{j(n-1)}, I=[0,1], \mathcal{F}^{\bullet, 0}=\Lambda \times_{M} \Lambda_{0}$ and $\mathcal{F}^{0, \bullet}=\Lambda_{0} \times_{M} \Lambda$. The mapping $\tau$ is the Thom isomorphism given by the cup product with the Thom class $\mu_{\mathcal{F}}$ of the normal bundle of $\phi_{\frac{1}{2}} \mathcal{F}$ in $\Lambda$, and $\kappa$ is given by the Künneth theorem. It uses the fact that $J\left(\Lambda_{0} \times[0,1]\right)$ and $J(\Lambda \times\{0,1\})$ are disjoint from $\phi_{\frac{1}{2}}\left(\mathcal{F}^{>0,>0}\right)$. 


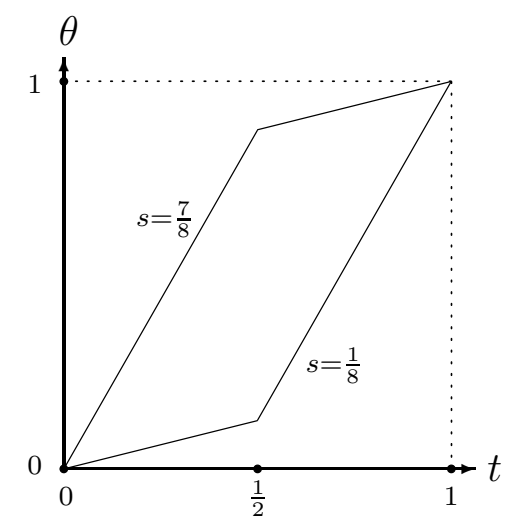

FiguRE 2. Graph of $\theta_{\frac{1}{2} \rightarrow s}$

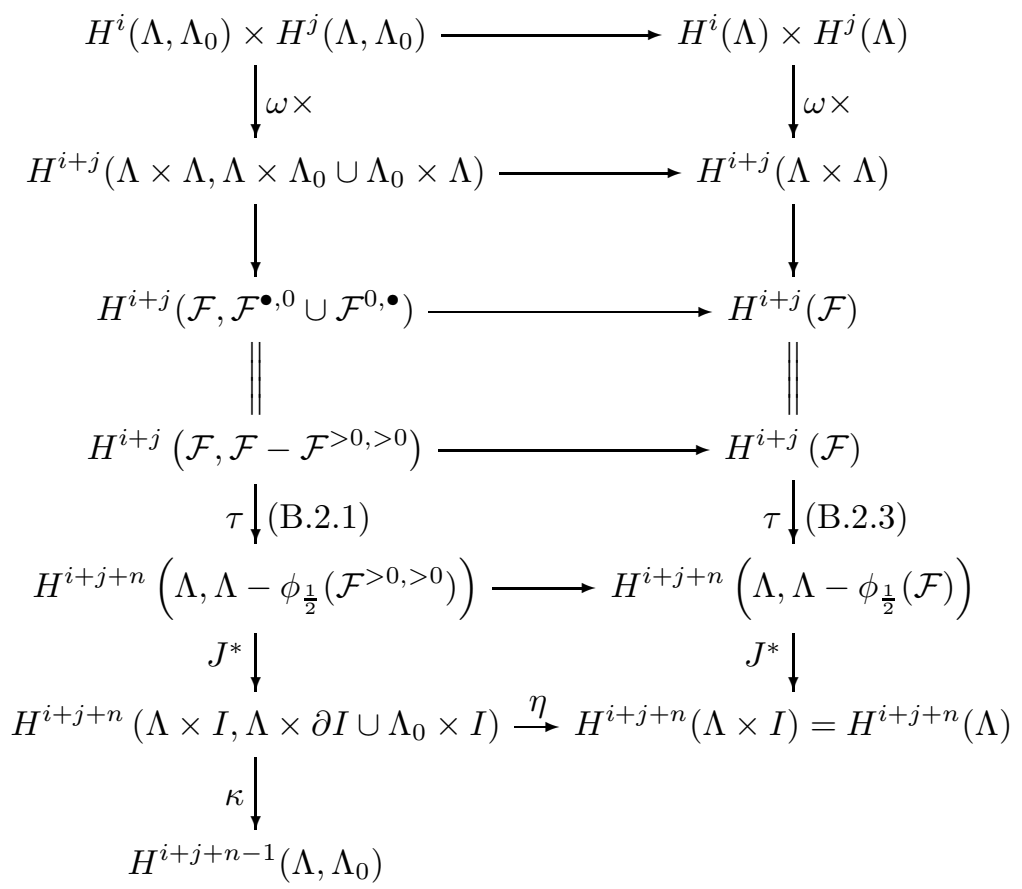

Figure 3. Definition of $\circledast$

Denote the cohomology product of two classes $\alpha, \beta \in H^{*}\left(\Lambda, \Lambda_{0}\right)$ by $\alpha \circledast \beta$. The construction may be summarized as passing from the left to the right in the following diagram.

$$
\Lambda \times \Lambda \longleftarrow \mathcal{F} \longrightarrow \Lambda \stackrel{J}{\longleftarrow} \Lambda \times I
$$

In Figure 3, the first horizontal mapping is an isomorphism if $i, j>n$ since $\Lambda_{0} \cong M$ has dimension $n$. The mapping $\eta$ is part of the long exact sequence for the pair $\left(\Lambda, \Lambda_{0}\right) \times(I, \partial I)$ 
so it is zero. It follows that the cohomology product defined in $₫ 8.1$, which is the composition down the right hand column, vanishes if $i, j>n$.

8.2. Remarks. Although the finite dimensional approximation $\mathcal{M}_{N}$ of Morse plays only a minor role in this paper, the cohomology product was first discovered as a product defined on the level cohomology of the subspace $\mathcal{A}_{N} \subset \mathcal{M}_{N}$, consisting of loops in $\mathcal{M}_{N}$ for which the $N$ pieces all have the same length, as follows. Using Poincaré duality, $H^{*}\left(\mathcal{A}_{N}^{\leq a}, \mathcal{A}_{N}^{<a}\right) \cong$ $H_{*}\left(\mathcal{A}_{N}^{\geq a}, \mathcal{A}_{N}^{>a}\right)$. Then apply the (homology) Chas-Sullivan product, followed by Poincaré duality again. Although the space $\mathcal{A}_{N}$ is a smooth retraction of $\mathcal{M}_{N}$ in a neighborhood of the critical set, it has singularities and (what is worse), spurious critical points, so it is preferable to work with $\mathcal{M}_{N}$. When the construction is translated to $\mathcal{M}_{N}$ it becomes the Chas-Sullivan product on Poincaré duals, followed by multiplication by a one parameter family of reparametrizations (the mapping $J$ ). This "level product" on $\mathcal{M}_{N}$ then extends to a product on $H^{*}\left(\mathcal{M}_{N}, \mathcal{M}_{N}^{=0}\right)$ that is independent of $N$, and hence it extends to a product on $H^{*}\left(\Lambda, \Lambda_{0}\right)$.

8.3. Proposition. The cohomology product $\circledast$ is associative. If $x \in H^{i}\left(\Lambda, \Lambda_{0}\right)$ and $y \in$ $H^{j}\left(\Lambda, \Lambda_{0}\right)$ then

$$
y \circledast x=(-1)^{(i+n-1)(j+n-1)} x \circledast y .
$$

Proof. First we prove that the product $\circledast$ is commutative. As in $\$ 5.3$ let $\sigma: \Lambda \times \Lambda \rightarrow \Lambda \times \Lambda$ switch the two factors and let $\widehat{\chi}_{r}: \Lambda \rightarrow \Lambda$ be the action of $r \in S^{1}$ given by $\widehat{\chi}_{r}(\gamma)=\gamma \circ \chi_{r}$ where $\chi_{r}(t)=r+t(\bmod 1)$. Then

$$
\chi_{1-s} \circ \theta_{\frac{1}{2} \rightarrow s}=\theta_{\frac{1}{2} \rightarrow(1-s)} \circ \chi_{\frac{1}{2}}
$$

(which may be seen from a direct calculation). For $0 \leq r \leq 1$ define $J_{r}: \Lambda \times I \rightarrow \Lambda$ by

$$
J_{r}(\gamma, s)=\gamma \circ \chi_{r(1-s)} \circ \theta_{\frac{1}{2} \rightarrow s}
$$

Then $J_{r}\left((\Lambda \times \partial I) \cup\left(\Lambda_{0} \times I\right)\right) \subset \Lambda-\phi_{\frac{1}{2}}\left(\mathcal{F}^{>0,>0}\right)$ because when $s=0$ or $s=1$ the loop $J_{r}(\gamma, s)$ stays fixed on either $\left[0, \frac{1}{2}\right]$ or $\left[\frac{1}{2}, 1\right]$. Therefore the mapping $J^{*}$ in Figure 3 may be replaced by $J_{r}^{*}$ for any $r \in[0,1]$. However, $J_{0}=J$ and

$$
\begin{aligned}
J_{1}(\gamma, s) & =\gamma \circ \chi_{1-s} \circ \theta_{\frac{1}{2} \rightarrow s}=\gamma \circ \theta_{\frac{1}{2} \rightarrow(1-s)} \circ \chi_{\frac{1}{2}} \\
& =\widehat{\chi}_{\frac{1}{2}}(J(\gamma, 1-s)) .
\end{aligned}
$$

This means that $J_{1}$ reverses the $s \in I$ coordinate and it switches the front and back half of any figure eight loop $\gamma \in \mathcal{F}$, cf. equation (5.3.1). Consequently, if $i: \mathcal{F} \rightarrow \Lambda \times \Lambda$ denotes 
the inclusion, we have:

$$
\begin{aligned}
(-1)^{i(n-1)} y \circledast x & =\kappa J_{0}^{*}\left(\mu_{\mathcal{F}} \cup i^{*}(y \times x)\right) \\
& =(-1)^{i j} \kappa J_{0}^{*}\left(\mu_{\mathcal{F}} \cup i^{*} \sigma^{*}(x \times y)\right) \\
& =(-1)^{i j}(-1)^{n} \kappa J_{0}^{*} \sigma^{*}\left(\mu_{\mathcal{F}} \cup i^{*}(x \times y)\right) \\
& =(-1)^{i j}(-1)^{n} \kappa J_{1}^{*} \sigma^{*}\left(\mu_{\mathcal{F}} \cup i^{*}(x \times y)\right) \\
& =(-1)^{i j}(-1)^{n}(-1) \kappa J_{0}^{*}\left(\mu_{\mathcal{F}} \cup i^{*}(x \times y)\right) \\
& =(-1)^{i j+n+1}(-1)^{j(n-1)} x \circledast y .
\end{aligned}
$$

giving equation (8.3.1). The proof of associativity can be found in Appendix E.

8.4. Based loop space and Pontrjagin product. Fix a base point $x_{0} \in M$ and let $\Omega=\Omega_{x_{0}}=\mathbf{e v}_{0}^{-1}\left(x_{0}\right)$ be the (based) loop space. It is a Hilbert submanifold of codimension $n=\operatorname{dim}(M)$ in $\Lambda$ with a trivial normal bundle. The Pontrjagin product

$$
\Omega \times \Omega \stackrel{\bullet}{\longrightarrow} \Omega
$$

(speed up by a factor of 2 and concatenate at time 1/2) is an embedding. Denote its image (which is the "based loops" analog of the figure eight space) by $\mathcal{F}_{\Omega}=\Omega \bullet \Omega$. It is a Hilbert submanifold of $\Omega$, with trivial $n$-dimensional normal bundle. We obtain a fiber (or "Cartesian") square,

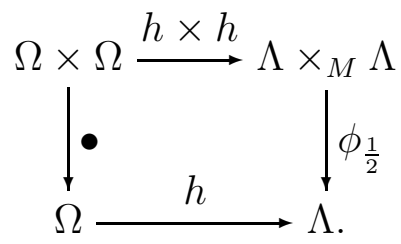

It follows that the Pontrjagin product (which is not necessarily commutative) and the ChasSullivan product are related ([CS] Prop. 3.4) by

$$
h^{!}(a * b)=h^{!}(a) \bullet h^{!}(b)
$$

for all $a, b \in H_{*}(\Lambda)$, where $h^{!}: H_{*}(\Lambda) \rightarrow H_{*-n}(\Omega)$ denotes the Gysin homomorphism (B.2.4).

A similar construction holds in cohomology. We use the same letter $J$ to denote its restriction, $J: \Omega \times I \rightarrow \Omega$ with $I=[0,1]$.

8.5. Proposition. Replacing $\left(\Lambda, \Lambda_{0}\right)$ by $\left(\Omega, x_{0}\right)$ in Figure 3 gives a product

$$
H^{i}\left(\Omega, x_{0}\right) \times H^{j}\left(\Omega, x_{0}\right) \stackrel{\circledast}{\longrightarrow} H^{i+j+n-1}\left(\Omega, x_{0}\right)
$$

such that

$$
h^{*}(a \circledast b)=\underset{23}{h^{*}(a) \circledast h^{*}(b)}
$$


for any $a \in H^{i}\left(\Lambda, \Lambda_{0}\right)$ and $b \in H^{j}\left(\Lambda, \Lambda_{0}\right)$. This product is often nontrivial. Suppose $X, Y \subset$ $\Omega$ are smooth compact oriented submanifolds of dimension $i, j$ respectively, and suppose $Z \subset \Omega$ is an oriented compact submanifold of dimension $i+j+n-1$ such that the mapping $J: Z \times I \rightarrow \Omega$ is transverse to $\mathcal{F}_{\Omega}=\Omega \bullet \Omega$ and such that

$$
J^{-1}\left(\mathcal{F}_{\Omega}\right)=(X \bullet Y) \times\left\{\frac{1}{2}\right\} .
$$

Then

$$
\langle a \circledast b, Z\rangle=\langle a,[X]\rangle \cdot\langle b,[Y]\rangle
$$

for any $a \in H^{i}\left(\Omega, x_{0}\right)$ and $b \in H^{j}\left(\Omega, x_{0}\right)$.

Proof. The nontriviality of the product will be taken up in $\$ 13.9$, The following diagram is a fiber (or "Cartesian") square so the Thom class of $X \bullet Y$ in $Z \times I$ is $J^{*}\left(\mu_{\mathcal{F}}\right)$ :

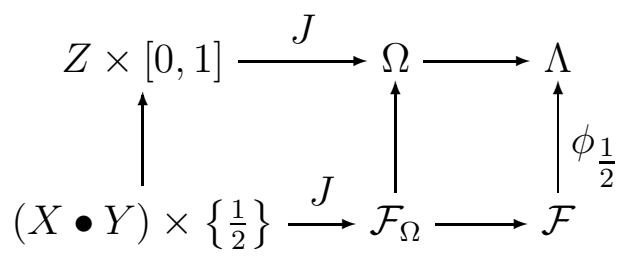

and the following diagram commutes.

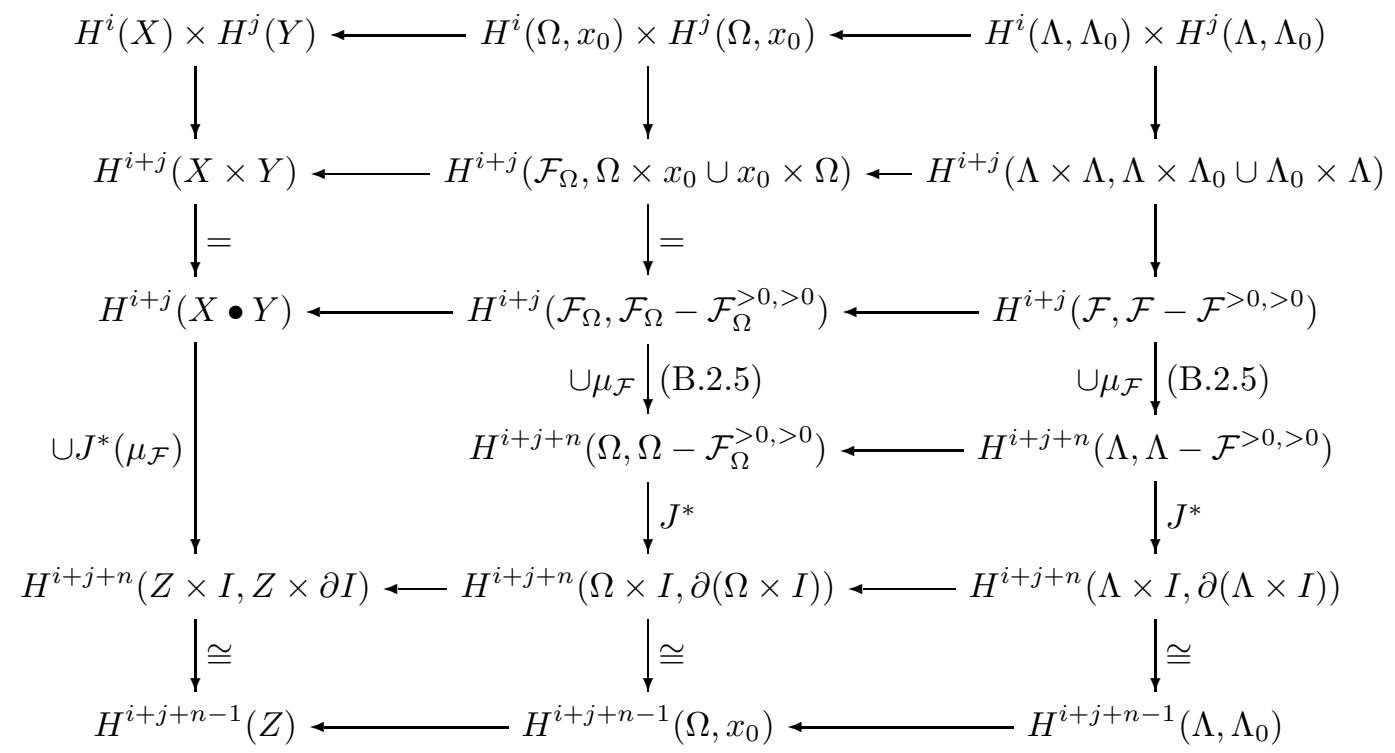

Here, $\partial(\Omega \times I)=x_{0} \times I \cup \Omega \times \partial I$ and $\partial(\Lambda \times I)=\Lambda_{0} \times I \cup \Lambda \times \partial I$. 
8.6. Proposition. Let $0 \leq a^{\prime}<a \leq \infty$ and $0 \leq b^{\prime}<b<\infty$. Then the cohomology product induces a family of compatible products

$$
\begin{aligned}
& H^{i}\left(\Lambda^{<a}, \Lambda^{<a^{\prime}}\right) \times H^{j}\left(\Lambda^{<b}, \Lambda^{<b^{\prime}}\right) \stackrel{\circledast}{\longrightarrow} H^{i+j+n-1}\left(\Lambda^{<c}, \Lambda^{<c^{\prime}}\right) \\
& \check{H}^{i}\left(\Lambda^{\leq a}, \Lambda^{\leq a^{\prime}}\right) \times \check{H}^{j}\left(\Lambda^{\leq b}, \Lambda^{\leq b^{\prime}}\right) \stackrel{\circledast}{\longrightarrow} \check{H}^{i+j+n-1}\left(\Lambda^{\leq c}, \Lambda^{\leq c^{\prime}}\right) \\
& \check{H}^{i}\left(\Lambda^{\leq a}, \Lambda^{<a}\right) \times \check{H}^{j}\left(\Lambda^{\leq b}, \Lambda^{<b}\right) \stackrel{\circledast}{\longrightarrow} \check{H}^{i+j+n-1}\left(\Lambda^{\leq a+b}, \Lambda^{<a+b}\right) .
\end{aligned}
$$

where $c=\min \left(a+b^{\prime}, a^{\prime}+b\right)$ and $c^{\prime}=a^{\prime}+b^{\prime}$. It is compatible with the homomorphisms induced by inclusions $\Lambda^{<e} \rightarrow \Lambda^{<f}$ whenever $e \leq f$.

The construction of the product (8.6.1) will be taken up in the next few sections. The existence of (8.6.2) and (8.6.3) follows from (8.6.1) and Appendix A.3.

8.7. For technical reasons it is easiest to construct this product using the space $\mathcal{A}$ of loops PPAL, rather than $\Lambda$. This choice allows us to work with the mapping $\phi_{\frac{1}{2}}$, which is an embedding, rather than more awkward mapping $\phi_{\min }$. It also allows us to keep track of the effect of the function $J$ on the energy; see (8.9.3). Recall (\$2.1) if $\alpha \in \mathcal{A}$ is a loop parametrized proportionally to arclength then $F(\alpha)=\sqrt{E(\alpha)}=L(\alpha)$ is its length. Let $\mathcal{F}_{\mathcal{A}}=\mathcal{A} \times{ }_{M} \mathcal{A}$ be the associated figure eight space consisting of pairs of composable loops, each parametrized proportionally to arclength. Let $\mathcal{A}_{\frac{1}{2}}$ be the set of loops $\alpha \in \Lambda$ such that $\alpha \mid[0,1 / 2]$ is PPAL and $\alpha \mid[1 / 2,1]$ is PPAL. Similarly let

$$
\begin{aligned}
& \mathcal{F}_{\mathcal{A}}^{<u,<v}=\left\{(\alpha, \beta) \in \mathcal{A} \times_{M} \mathcal{A}: L(\alpha)<u \text { and } L(\beta)<v .\right\} \\
& \mathcal{A}_{\frac{1}{2}}^{<u,<v}=\left\{\alpha \in \mathcal{A}_{\frac{1}{2}}: L(\alpha \mid[0,1 / 2])<u \text { and } L(\alpha \mid[1 / 2,1]<v .\}\right.
\end{aligned}
$$

and similarly for $\mathcal{A}_{\frac{1}{2}}^{=u,=v}$, etc. Then the mapping $\phi_{\frac{1}{2}}$ restricts to a closed embedding

$$
\phi_{\frac{1}{2}}: \mathcal{F}_{\mathcal{A}}^{<u,<v} \rightarrow \mathcal{A}_{\frac{1}{2}}^{<u,<v}
$$

8.8. Lemma. The orientation of $M$ induces a Thom isomorphism

$$
H^{i}\left(\mathcal{F}_{\mathcal{A}}^{<u,<v}\right) \cong H^{i+n}\left(\mathcal{A}_{\frac{1}{2}}^{<u,<v}, \mathcal{A}_{\frac{1}{2}}^{<u,<v}-\phi_{\frac{1}{2}}\left(\mathcal{F}_{\mathcal{A}}^{<u,<v}\right)\right)
$$

for the image, $\phi_{\frac{1}{2}}\left(\mathcal{F}_{\mathcal{A}}^{<u,<v}\right)$. If $Z \subset \mathcal{F}_{\mathcal{A}}^{<u,<v}$ is a closed subset then this restricts to a relative Thom isomorphism,

$$
H^{i}\left(\mathcal{F}_{\mathcal{A}}^{<u,<v}, \mathcal{F}_{\mathcal{A}}^{<u,<v}-Z\right) \cong H^{i+n}\left(\mathcal{A}_{\frac{1}{2}}^{<u,<v}, \mathcal{A}_{\frac{1}{2}}^{<u,<v}-Z\right) .
$$

Proof. The space $\phi_{\frac{1}{2}}\left(\mathcal{F}_{\mathcal{A}}^{<u,<v}\right)$ may be described as the pre-image of the diagonal under the mapping

$$
\left(\mathbf{e v}_{0}, \mathbf{e v}_{\frac{1}{2}}\right): \mathcal{A}_{\frac{1}{2}}^{<u,<v} \rightarrow M \times M
$$


If $\mathcal{A}_{\frac{1}{2}}$ were a Hilbert manifold, this would imply the existence of a normal bundle and tubular neighborhood for $\phi_{\frac{1}{2}}\left(\mathcal{F}_{\mathcal{A}}^{<u,<v}\right)$ in $\mathcal{A}_{\frac{1}{2}}^{<u,<v}$. Unfortunately $\mathcal{A}_{\frac{1}{2}}$ is probably not a Hilbert manifold, and even though it may be a Banach manifold, we do not know of any standard reference for the existence of tubular neighborhoods which can be applied in this setting. However we have the following commutative diagram, where the vertical maps are homotopy equivalences, cf. Proposition 2.2 ,

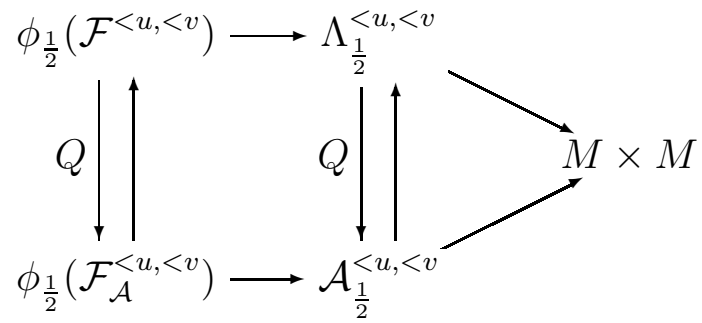

Here, $\Lambda_{\frac{1}{2}}^{<u,<v}$ denotes the set of all $\alpha \in \Lambda$ such that $E\left(\alpha_{1}\right)<u^{2}$ and $E\left(\alpha_{2}\right)<v^{2}$ where $\alpha_{1}(t)=\alpha(2 t)(0 \leq t \leq 1 / 2)$ and $\alpha_{2}(t)=\alpha(2 t-1)(1 / 2 \leq t \leq 1)$. (In other words, if $\alpha \mid[0,1 / 2]$ and $\alpha \mid[1 / 2,1]$ are both expanded into paths defined on $[0,1]$ then their respective energies are bounded by $u^{2}$ and $v^{2}$.) Since $\Lambda$ is a Hilbert manifold the same holds for $\Lambda_{\frac{1}{2}}^{<u,<v}$ hence $\phi_{\frac{1}{2}}\left(\mathcal{F}^{<u,<v}\right)$ has a tubular neighborhood and normal bundle in $\Lambda^{<u,<v}$ and we have a Thom isomorphism

$$
H^{i}\left(\mathcal{F}^{<u,<v}\right) \cong H^{i+n}\left(\Lambda^{<u,<v}, \Lambda^{<u,<v}-\phi_{\frac{1}{2}}\left(\mathcal{F}^{<u,<v}\right)\right) .
$$

The vertical homotopy equivalence in this diagram assigns to any $\alpha \in \Lambda_{\frac{1}{2}}^{<u,<v}$ the same curve but with $\alpha \mid[0,1 / 2]$ reparametrized proportionally to arclength and with $\alpha \mid[1 / 2,1]$ similarly reparametrized. It restricts to a homotopy equivalences $\phi_{\frac{1}{2}}\left(\mathcal{F}^{<u,<v}\right) \rightarrow \phi_{\frac{1}{2}}\left(\mathcal{F}_{\mathcal{A}}^{<u,<v}\right)$ and also

$$
\Lambda_{\frac{1}{2}}^{<u,<v}-\phi_{\frac{1}{2}}\left(\mathcal{F}^{<u,<v}\right) \rightarrow \mathcal{A}_{\frac{1}{2}}^{<u,<v}-\phi_{\frac{1}{2}}\left(\mathcal{F}_{\mathcal{A}}^{<u,<v}\right)
$$

because the points $\alpha(0), \alpha(1 / 2)$ are fixed. The Thom isomorphism (8.8.1) follows. The relative Thom isomorphism (8.8.2) follows as in Proposition B.2.

8.9. Let $I=[0,1]$ denote the unit interval. The mapping $J: \Lambda \times I \rightarrow \Lambda$ restricts to a mapping $J_{\mathcal{A}}: \mathcal{A} \times I \rightarrow \mathcal{A}_{\frac{1}{2}}$ which factors through the quotient $\mathcal{A} \times I /\left(\mathcal{A}_{0} \times I\right)$ that is obtained from $\mathcal{A} \times I$ by identifying $\mathcal{A}_{0} \times I$ to a point. The resulting mapping

$$
\mathcal{A} \times I /\left(\mathcal{A}_{0} \times I\right) \rightarrow \mathcal{A}_{\frac{1}{2}}
$$

is a homeomorphism, and in fact the inverse mapping can be described as follows. Let $\alpha \in \mathcal{A}_{\frac{1}{2}}-\mathcal{A}_{0}$. Let $L_{0}$ denote the length of the segment $\alpha \mid\left[0, \frac{1}{2}\right]$ (which is PPAL) and let $L_{1}$ denote the length of the segment $\alpha \mid\left[\frac{1}{2}, 1\right]$. Set $s=L_{0} /\left(L_{0}+L_{1}\right)$. Assume for the moment that $0<s<1$. Let $\theta_{s \rightarrow \frac{1}{2}}=\left(\theta_{\frac{1}{2} \rightarrow s}\right)^{-1}$ be the inverse function to $\theta_{\frac{1}{2} \rightarrow s}$; it is linear on $[0, s]$, 
linear on $[s, 1]$, and takes the values $\theta_{s \rightarrow \frac{1}{2}}(0)=0, \theta_{s \rightarrow \frac{1}{2}}(s)=\frac{1}{2}, \theta_{s \rightarrow \frac{1}{2}}(1)=1$. Then $\alpha \circ \theta_{s \rightarrow \frac{1}{2}}$ is PPAL throughout the interval $[0,1]$ so we may set

$$
J_{\mathcal{A}}^{-1}(\alpha)=\left(\alpha \circ \theta_{s \rightarrow \frac{1}{2}}, s\right) .
$$

If $s=0$ (resp. $s=1$ ) then this formula still makes sense because in this case, the loop $\alpha$ will be constant on $\left[0, \frac{1}{2}\right]$ (resp. on $\left[\frac{1}{2}, 1\right]$ ). If $\alpha_{0}, \alpha_{1} \in \mathcal{A}$ are composable loops, not both constant, then the composed loop $\phi_{\frac{1}{2}}\left(\alpha_{0}, \alpha_{1}\right) \in \mathcal{A}_{\frac{1}{2}}$ and

$$
J_{\mathcal{A}}^{-1}\left(\phi_{\frac{1}{2}}\left(\alpha_{0}, \alpha_{1}\right)\right)=\left(\phi_{s}\left(\alpha_{0}, \alpha_{1}\right), s\right)=\left(\phi_{\min }\left(\alpha_{0}, \alpha_{1}\right), s\right)
$$

where $s=L\left(\alpha_{0}\right) /\left(L\left(\alpha_{0}\right)+L\left(\alpha_{1}\right)\right)$ is the unique energy minimizing value, cf. Lemma 2.4.

It follows that if $A, B \subset \mathcal{A}$ then the mapping $J_{\mathcal{A}}: \mathcal{A} \times[0,1] \rightarrow \mathcal{A}_{\frac{1}{2}}$ takes

$$
(\mathcal{A}-A * B) \times[0,1] \text { into } \mathcal{A}_{\frac{1}{2}}-\phi_{\frac{1}{2}}\left(A \times_{M} B\right) .
$$

For $J\left(\mathcal{A}_{0} \times[0,1]\right)=\mathcal{A}_{0}$ which is contained in the right hand side, so it suffices to check that $J(A * B \times[0,1]) \supset \phi_{\frac{1}{2}}\left(A \times_{M} B\right)$, which follows from (8.9.1).

Similarly the mapping $J_{\mathcal{A}}$ satisfies

$$
J_{\mathcal{A}}(\alpha, s) \in \mathcal{A}_{\frac{1}{2}}^{=s L(\alpha),=(1-s) L(\alpha)}
$$

which means the following: if we express $J_{\mathcal{A}}(\alpha, s)=\phi_{\frac{1}{2}}\left(\beta_{1}, \beta_{2}\right)$ as a composition of two (not necessarily closed) paths $\beta_{1}, \beta_{2}$, each PPAL, and joined at time $1 / 2$, then $L\left(\beta_{1}\right)=s L(\alpha)$ and $L\left(\beta_{2}\right)=(1-s) L(\alpha)$.

8.10. Define

$$
\begin{aligned}
T^{<a,<b} & =J_{\mathcal{A}}^{-1}\left(\mathcal{F}_{\mathcal{A}}^{<a,<b}\right) \\
& =\{(\alpha, s) \in \mathcal{A} \times I: s L(\alpha)<a \text { and }(1-s) L(\alpha)<b\} \\
T^{\left[a^{\prime}, a\right),\left[b^{\prime}, b\right)} & =J_{\mathcal{A}}^{-1}\left(\mathcal{F}_{\mathcal{A}}^{\left[a^{\prime}, a\right),\left[b^{\prime}, b\right)}\right) \\
& =\left\{(\alpha, s) \in \mathcal{A}_{\frac{1}{2}} \times I: a^{\prime} \leq s L(\alpha)<a, \text { and } b^{\prime} \leq(1-s) L(\alpha)<b\right\} .
\end{aligned}
$$

Figure 8.10 consists of three diagrams of $L=\sqrt{E}$ versus $s \in[0,1]$ illustrating the curves $s L=a$ and $(1-s) L=b$ that occur in the definition of $T^{<a,<b}$. These curves intersect at the point with coordinates $s=a /(a+b)$ and $L=a+b$. The diagrams on the right illustrate the corresponding regions for $T^{\left[a^{\prime} \cdot a\right),\left[b^{\prime}, b\right)}$. (The interval $I^{\prime}$ is defined in the next paragraph, §.11.) 

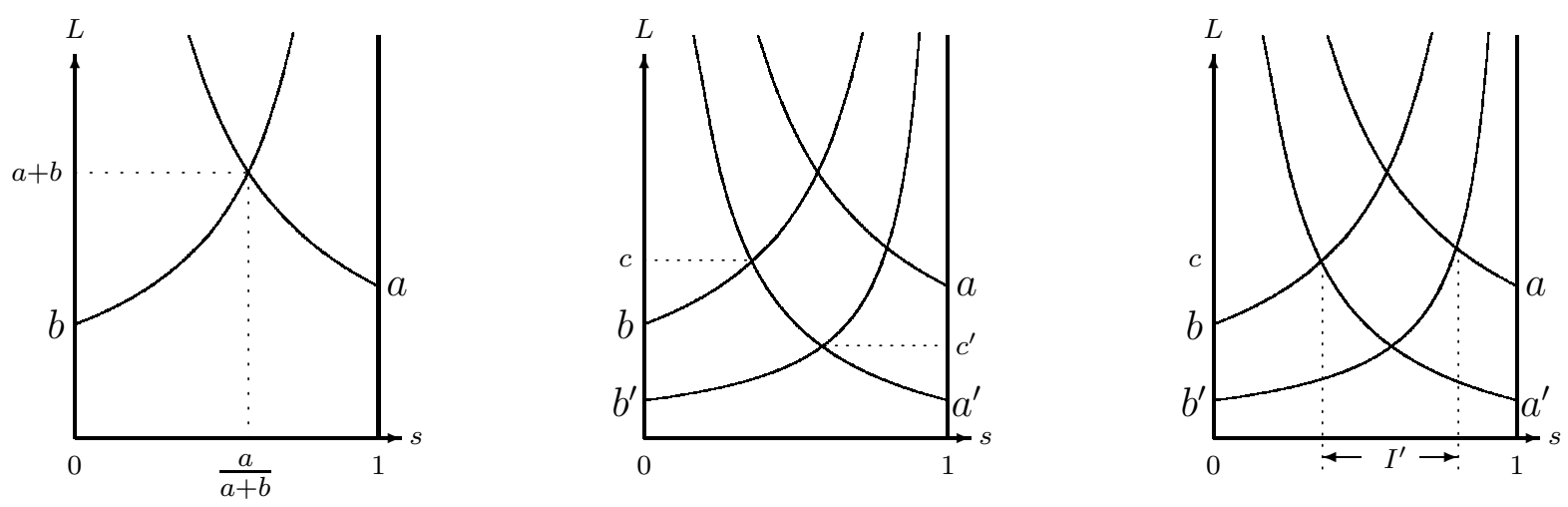

FiguRe 4. The regions $T^{<a,<b}$ and $T^{\left[a^{\prime}, a\right),\left[b^{\prime}, b\right)}$

8.11. The product (8.6.1) is constructed in several steps. First, use the cross product,

$$
H^{i}\left(\mathcal{A}^{<a}, \mathcal{A}^{<a^{\prime}}\right) \times H^{j}\left(\mathcal{A}^{<b}, \mathcal{A}^{<b^{\prime}}\right) \rightarrow H^{i+j}\left(\mathcal{A}^{<a} \times \mathcal{A}^{<b}, \mathcal{A}^{<a} \times \mathcal{A}^{<b^{\prime}} \cup \mathcal{A}^{<a^{\prime}} \times \mathcal{A}^{<b}\right)
$$

then restrict to

$$
H^{i+j}\left(\mathcal{F}_{\mathcal{A}}^{<a,<b}, \mathcal{F}_{\mathcal{A}}^{<a,<b^{\prime}} \cup \mathcal{F}_{\mathcal{A}}^{<a^{\prime},<b}\right)=H^{i+j}\left(\mathcal{F}_{\mathcal{A}}^{<a,<b}, \mathcal{F}_{\mathcal{A}}^{<a,<b}-\mathcal{F}_{\mathcal{A}}^{\left[a^{\prime}, a\right),\left[b^{\prime}, b\right)}\right) .
$$

Using the embedding (8.7.1) and the Thom isomorphism we arrive at

$$
\begin{aligned}
& H^{i+j}\left(\phi_{\frac{1}{2}}\left(\mathcal{F}_{\mathcal{A}}^{<a,<b}\right), \phi_{\frac{1}{2}}\left(\mathcal{F}_{\mathcal{A}}^{<a,<b}\right)-\phi_{\frac{1}{2}}\left(\mathcal{F}_{\mathcal{A}}^{\left[a^{\prime}, a\right),\left[b^{\prime}, b\right)}\right)\right) \\
\cong & H^{i+j+n}\left(\mathcal{A}_{\frac{1}{2}}^{<a,<b}, \mathcal{A}_{\frac{1}{2}}^{<a,<b}-\phi_{\frac{1}{2}}\left(\mathcal{F}_{\mathcal{A}}^{\left[a^{\prime}, a\right),\left[b^{\prime}, b\right)}\right)\right) .
\end{aligned}
$$

Pulling back under $J_{\mathcal{A}}$ gives a class in

$$
H^{i+j+n}\left(T^{<a,<b}, T^{<a,<b}-T^{\left[a^{\prime}, a\right),\left[b^{\prime}, b\right)} .\right)
$$

Let $I^{\prime}$ denote the interval $\left[\frac{a^{\prime}}{a^{\prime}+b}, \frac{a}{a+b^{\prime}}\right]$. Then $\mathcal{A}^{<c} \times I^{\prime} \subset T^{<a,<b}$ and

$$
\left(\mathcal{A}^{<c} \times \partial I^{\prime}\right) \cup\left(\mathcal{A}^{<c^{\prime}} \times I^{\prime}\right) \subset T^{<a .<b}-T^{\left[a^{\prime}, a\right),\left[b^{\prime}, b\right)}
$$

where $c=\min \left(a+b^{\prime}, a^{\prime}+b\right)$ and $c^{\prime}=a^{\prime}+b^{\prime}$. In other words, the mapping $J_{\mathcal{A}}$ restricts to a map of pairs,

$$
J_{\mathcal{A}}:\left(\mathcal{A}^{<c}, \mathcal{A}^{<c^{\prime}}\right) \times\left(I^{\prime}, \partial I^{\prime}\right) \rightarrow\left(\phi_{\frac{1}{2}}\left(\mathcal{F}_{\mathcal{A}}^{<a,<b}\right), \phi_{\frac{1}{2}}\left(\mathcal{F}_{\mathcal{A}}^{<a,<b}\right)-\phi_{\frac{1}{2}}\left(\mathcal{F}_{\mathcal{A}}^{\left[a^{\prime}, a\right),\left[b^{\prime}, b\right)}\right)\right)
$$

Therefore the class in (8.11.1) pulls back to a class in

$$
H^{i+j+n}\left(\left(\mathcal{A}^{<c}, \mathcal{A}^{<c^{\prime}}\right) \times\left(I^{\prime}, \partial I^{\prime}\right)\right) \cong H^{i+j+n-1}\left(\mathcal{A}^{<c}, \mathcal{A}^{<c^{\prime}}\right)
$$

as claimed. This completes the proof of Proposition 8.6

Taking $a, b=\infty$ and $a^{\prime}, b^{\prime}=0$ gives an equivalent construction of the cohomology product $\circledast$ using the space $\mathcal{A}$ rather than $\Lambda$. This will also be important in the next section. 


\section{Support and critical levels}

9.1. As in the previous sections, we take cohomology with coefficients in $G=\mathbb{Z}$ if $M$ is orientable, or $G=\mathbb{Z} /(2)$ otherwise, but we suppress mention of $G$ in our notation for cohomology. Proposition 8.6 gives:

9.2. Proposition. If $\alpha, \beta \in H^{*}\left(\Lambda, \Lambda_{0}\right)$ then $\operatorname{cr}(\alpha \circledast \beta) \geq \operatorname{cr}(\alpha)+\operatorname{cr}(\beta)$.

As in $\$ 2.1$ and $\$ 8.7$ let $\mathcal{A}$ be the set of loops parametrized proportionally to arclength, let $\mathcal{A}_{0}=\Lambda_{0}$ be the constant loops and let $\mathcal{A}_{\frac{1}{2}} \subset \Lambda$ be the collection of those loops which are PPAL on $[0,1 / 2]$ and are PPAL on $[1 / 2,1]$. We have continuous mappings

$$
J_{\mathcal{A}}: \mathcal{A} \times[0,1] \rightarrow \mathcal{A}_{\frac{1}{2}}, \quad \phi_{\frac{1}{2}}: \mathcal{A} \times_{M} \mathcal{A} \rightarrow \mathcal{A}_{\frac{1}{2}}, \quad \text { and } \phi_{\min }: \mathcal{A} \times_{M} \mathcal{A} \rightarrow \mathcal{A} \subset \mathcal{A}_{\frac{1}{2}} .
$$

9.3. Proposition. Suppose $\alpha \in H^{i}\left(\mathcal{A}, \mathcal{A}_{0}\right)$ is supported on a closed set $A \subset \mathcal{A}-\mathcal{A}_{0}$. Suppose $\beta \in \check{H}^{j}\left(\mathcal{A}, \mathcal{A}_{0}\right)$ is supported on a closed set $B \subset \mathcal{A}-\mathcal{A}_{0}$. Then $\alpha \circledast \beta$ is supported on the closed set $A * B=\phi_{\min }\left(A \times_{M} B\right) \subset \mathcal{A}-\mathcal{A}_{0}$.

Proof. Using (8.9.2) we obtain a cohomology product

$$
H^{i}(\mathcal{A}, \mathcal{A}-A) \times H^{j}(\mathcal{A}, \mathcal{A}-B) \rightarrow H^{i+j+n-1}(\mathcal{A}, \mathcal{A}-A * B)
$$

as the composition

$$
\begin{aligned}
& H^{i+j}(\mathcal{A} \times \mathcal{A}, \mathcal{A} \times \mathcal{A}-A \times B) \longrightarrow H^{i+j}\left(\mathcal{F}_{\mathcal{A}}, \mathcal{F}_{\mathcal{A}}-A \times_{M} B\right) \\
& \text { (8.8.2) } \downarrow \phi_{\frac{1}{2}} \\
& H^{i+j+n}((\mathcal{A}, \mathcal{A}-A * B) \times(I, \partial I)) \stackrel{J_{\mathcal{A}}^{*}}{\longleftarrow} H^{i+j+n}\left(\mathcal{A}_{\frac{1}{2}}, \mathcal{A}_{\frac{1}{2}}-\phi_{\frac{1}{2}}\left(A \times_{M} B\right)\right)
\end{aligned}
$$

where $I=[0,1]$.

9.4. We remark that the analogous statement for the cup product in cohomology says that $\alpha \smile \beta$ is supported on the intersection $A \cap B$. In particular, for any $0 \leq a^{\prime}<a \leq \infty$ and $0 \leq b^{\prime}<b \leq \infty$ the cup product gives mappings (with coefficients in $\mathbb{Z}$ ),

$$
H^{i}\left(\Lambda^{\leq a}, \Lambda^{\leq a^{\prime}}\right) \times H^{j}\left(\Lambda^{\leq b}, \Lambda^{\leq b^{\prime}}\right) \rightarrow H^{i+j}\left(\Lambda^{\leq \min (a, b)}, \Lambda^{\leq \max \left(a^{\prime}, b^{\prime}\right)}\right) .
$$

9.5. On a question of Eliashberg. In a lecture at Princeton University in June 2007, Y. Eliashberg asked the following question. Let $M$ be a smooth compact Riemannian manifold and let $\Lambda$ be its free loop space. Given $0<t \in \mathbb{R}$ let $d(t)$ be the maximal degree of an essential homology class at level $t$, that is,

$$
d(t)=\max \left\{k: \operatorname{Image}\left(H_{k}\left(\Lambda^{\leq t}, \mathbb{Q}\right) \rightarrow H_{k}(\Lambda ; \mathbb{Q})\right) \neq 0\right\} .
$$

Does there exist a constant $C \in \mathbb{R}$, independent of the metric, so that for all $t_{1}, t_{2} \in \mathbb{R}^{+}$the following holds:

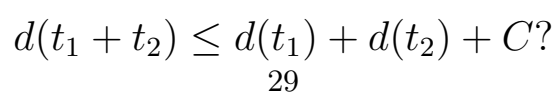


Inequalities in the opposite direction are known. If the cohomology $\operatorname{ring}\left(H^{*}\left(\Lambda, \Lambda_{0} ; \mathbb{Q}\right), \circledast\right)$ is finitely generated, then we are able to give an affirmative answer to this question. Moreover, in $\$ 13$ we show that the cohomology ring (with rational coefficients) is indeed finitely generated if $M$ is orientable and admits a metric in which all geodesics are closed. (If the orientability condition is dropped then the cohomology ring with $\mathbb{Z} /(2)$ coefficients is finitely generated.) This includes the case of spheres and projective spaces.

9.6. Theorem. Let $M$ be a smooth Riemannian $n$-dimensional manifold. Fix a coefficient field $G$, and for $t \in \mathbb{R}^{+}$define $d(t)=d(t ; G)$ by (9.5.1), but replacing the coefficients $\mathbb{Q}$ with the field $G$. Assume the cohomology ring $\left(H^{*}\left(\Lambda, \Lambda_{0} ; G\right), \circledast\right)$ is finitely generated, with all generators having degree $\leq g$. If $t_{1}, t_{2} \in \mathbb{R}^{+}$then

$$
d\left(t_{1}+t_{2}\right) \leq d\left(t_{1}\right)+d\left(t_{2}\right)+2 n+g-2 .
$$

Proof. Let $t_{1}, t_{2}, t_{3} \in \mathbb{R}^{+}$and let $d_{i}=d\left(t_{i} ; G\right)$ for $i=1,2,3$. We will show: if

$$
d_{3}>d_{1}+d_{2}+2 n+g-2
$$

then $t_{3}>t_{1}+t_{2}$. From the definition (9.5.1) and since $d_{3}>n=\operatorname{dim}\left(\Lambda_{0}\right)$, there exist non-zero homology classes $z^{\prime}$ and $z$, with:

$$
\begin{array}{r}
H_{d_{3}}\left(\Lambda^{\leq t_{3}}, \Lambda_{0}\right) \underset{i_{*}}{\longrightarrow} H_{d_{3}}\left(\Lambda, \Lambda_{0}\right) \\
z^{\prime} \longrightarrow=i_{*}\left(z^{\prime}\right)
\end{array}
$$

Let $Z \in H^{d_{3}}\left(\Lambda, \Lambda_{0}\right)$ be a cohomology class with non-zero Kronecker product,

$$
\langle Z, z\rangle \neq 0 \text {. }
$$

The class $Z$ is a sum of products of generators of the ring $\left(H^{*}\left(\Lambda, \Lambda_{0}\right), \circledast\right)$, and at least one term in this sum has a non-zero Kronecker product with $z$. Replacing $Z$ by this term, it may be expressed as a product of generators,

$$
Z=U_{1} \circledast U_{2} \circledast \cdots \circledast U_{q}
$$

with each $\operatorname{deg}\left(U_{i}\right) \leq g$.

We claim there exist $X, Y \in H^{*}\left(\Lambda, \Lambda_{0}\right)$ such that $Z=X \circledast Y$, with $\operatorname{deg}(X) \geq d_{1}+1$ and $\operatorname{deg}(Y) \geq d_{2}+1$. For, choose $p$ so that

$$
\begin{aligned}
\operatorname{deg}\left(U_{1} \circledast U_{2} \circledast \cdots \circledast U_{p-1}\right) & \leq d_{1} \\
\operatorname{deg}\left(U_{1} \circledast U_{2} \circledast \cdots \circledast U_{p}\right) & \geq d_{1}+1
\end{aligned}
$$

Take $X=U_{1} \circledast \cdots \circledast U_{p}$ and $Y=U_{p+1} \circledast \cdots \circledast U_{q}$. Then $\operatorname{deg}\left(U_{p}\right) \leq g \operatorname{sodeg}(X) \leq d_{1}+g+n-1$, while $\operatorname{deg}(X \circledast Y)=d_{3}>d_{1}+d_{2}+2 n+g-2$, so $\operatorname{deg}(Y)>d_{2}$.

Using this claim and (9.5.1), and setting $j=\operatorname{deg}(X)$ and $k=\operatorname{deg}(Y)$, there exists $\widehat{X}$ which maps to $X$ in the following exact sequence,

$$
\begin{gathered}
H^{j}\left(\Lambda, \Lambda^{\leq t_{1}}\right) \longrightarrow H^{j}\left(\Lambda, \Lambda_{0}\right) \longrightarrow H^{j}\left(\Lambda^{\leq t_{1}}, \Lambda_{0}\right) \\
\widehat{X} \longrightarrow \underset{30}{X}
\end{gathered}
$$


Similarly the class $Y$ has some lift $\widehat{Y} \in H^{k}\left(\Lambda, \Lambda \leq t_{2}\right)$. Then $\widehat{X} \circledast \widehat{Y} \in H^{d_{3}}\left(\Lambda, \Lambda^{t_{1}+t_{2}}\right)$ maps to $Z$. But this implies that $t_{3}>t_{1}+t_{2}$. Otherwise, $\Lambda^{\leq t_{3}} \subset \Lambda^{\leq t_{1}+t_{2}}$ so in the following diagram,

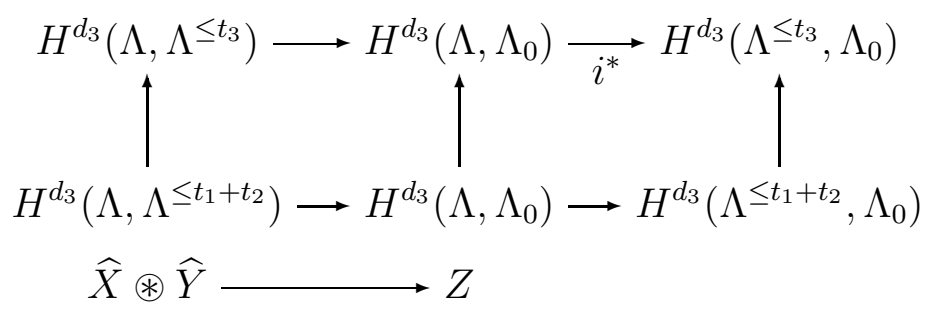

we would have $\langle Z, z\rangle=\left\langle Z, i_{*}\left(z^{\prime}\right)\right\rangle=\left\langle i^{*}(Z), z^{\prime}\right\rangle=0$ which contradicts (9.6.3).

\section{Level nilpotence for cohomology}

10.1. We say that a class $\alpha \in H^{i}\left(\Lambda, \Lambda_{0}\right)$ is level nilpotent if there exists $m$ so that $\operatorname{cr}\left(\alpha^{\circledast m}\right)>$ $m \operatorname{cr}(\alpha)$. We say that a class $\beta \in \check{H}^{i}\left(\Lambda^{\leq a}, \Lambda^{<a}\right)$ is level nilpotent if there exists $m$ so that $\beta^{\circledast m}=0$ in $\check{H}^{m i+(m-1)(n-1)}\left(\Lambda^{\leq m a}, \Lambda^{<m a}\right)$.

Let us say that two classes $\alpha \in H^{i}\left(\Lambda, \Lambda_{0}\right)$ and $\beta \in \check{H}^{i}\left(\Lambda \leq a, \Lambda^{<a}\right)$ are associated if there exists an associating class $\omega \in \check{H}^{i}\left(\Lambda, \Lambda^{<a}\right)$ with

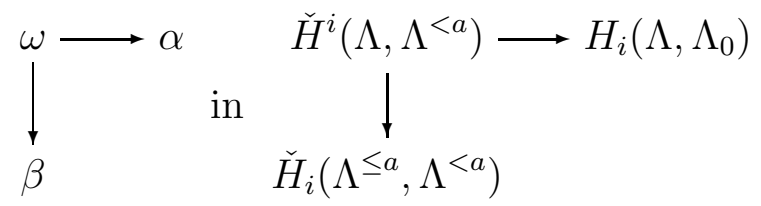

Then $\operatorname{cr}(\alpha)>a$ if and only if $\alpha$ is associated to the zero class $\beta=0 \in \check{H}^{i}\left(\Lambda^{\leq a}, \Lambda^{<a}\right)$.

10.2. Lemma. Suppose $\alpha \in H^{i}\left(\Lambda, \Lambda_{0}\right)$ and $\beta \in \check{H}^{i}\left(\Lambda^{\leq a}, \Lambda^{<a}\right)$ are associated, where $a=$ $\operatorname{cr}(\alpha)$. If $\beta$ is level nilpotent, then $\alpha$ is also level-nilpotent.

Proof. The proof is exactly parallel to that of Lemma 7.2

10.3. Theorem. Let $M$ be a compact $n$ dimensional Riemannian manifold and suppose that all critical points of the function $F=\sqrt{E}: \Lambda \rightarrow \mathbb{R}$ are nondegenerate (i.e. they lie on isolated nondegenerate critical orbits). If $M$ is orientable let $G=\mathbb{Z}$, otherwise let $G=\mathbb{Z} /(2)$. Then every class $\alpha \in H^{i}\left(\Lambda, \Lambda_{0} ; G\right)$ is level-nilpotent and every class $\beta \in H^{i}\left(\Lambda^{\leq a}, \Lambda^{<a} ; G\right)$ is level nilpotent (for any $i>0$ and any $a \in \mathbb{R}$ ).

Proof. The proof is similar to that of Theorem 7.3 .

\section{Level products in the nondegenerate case}

11.1. Throughout this section homology and cohomology will be taken with coefficients in $G=\mathbb{Z}$. Let $\Sigma \subset \Lambda$ be a nondegenerate critical orbit of index $\lambda$ and let $U \subset \Lambda$ be a 
sufficiently small neighborhood of $\sigma$. Assume the negative bundle $\Gamma \rightarrow \Sigma$ is orientable. Then the (local, level) homology groups are

$$
\check{H}_{i}\left(\Lambda^{<c} \cup \Sigma, \Lambda^{<c}\right) \cong H_{i}\left(\Lambda^{\leq c} \cap U, \Lambda^{<c} \cap U\right) \cong \begin{cases}\mathbb{Z} & \text { if } i=\lambda, \lambda+1 \\ 0 & \text { otherwise }\end{cases}
$$

and the same holds for the cohomology groups $H^{i}\left(\Lambda^{\leq c} \cap U, \Lambda^{<c} \cap U\right)$.

11.2. Now suppose $\gamma \in \Lambda$ is a prime geodesic, all of whose iterates are nondegenerate. Let $a$ be its length. Let $\gamma^{r}$ denote the $r$-fold iterate; $\lambda_{r}$ its Morse index; and let $\Sigma_{r} \subset \Lambda$ be its $S^{1}$-saturation. Assume the negative bundle $\Gamma_{r}$ over $\Sigma_{r}$ is orientable 3 , and let $\sigma_{r}, \bar{\tau}_{r}, \bar{\sigma}_{r}, \tau_{r}$ be generators for the local level (co)homology classes, that is,

$$
\sigma_{r} \in H_{\lambda_{r}}, \quad \bar{\sigma}_{r} \in H_{\lambda_{r}+1}, \quad \bar{\tau}_{r} \in H^{\lambda_{r}}, \quad \tau_{r} \in H^{\lambda_{r}+1} .
$$

As a consequence of the nilpotence results from $\$ 7$ and $\$ 10$, the index $\lambda_{r}$ can be neither minimal nor maximal for all $r$ (in the language of Proposition 6.1), and the local level homology and cohomology rings

$$
\left(\oplus H_{i}\left(\Lambda^{<a r} \cup \Sigma_{r}, \Lambda^{<a r} ; G\right), *\right) \quad \text { and } \quad\left(\oplus H^{i}\left(\Lambda^{<a r} \cup \Sigma_{r}, \Lambda^{<a r} ; G\right), \circledast\right)
$$

are not finitely generated. However, , if the index growth is minimal up to the $n$-th iterate (with $\lambda_{r}=\lambda_{r}^{\min }$ for all $r \leq n$ ) then nontrivial (level) homology products exist, and if the index growth is maximal up to the $2 n$-th iterate (with $\lambda_{r}=\lambda_{r}^{\max }$ for all $r \leq 2 n$ ) then nontrivial (level) cohomology products exist, as described in the following theorem.

11.3. Theorem. Assume the manifold $M$ is orientable and the negative bundle $\Gamma_{r}$ is orientable for all $r$. Assume $r \geq 2$. Then the following statements hold in the local level (co)homology group $H\left(\Lambda^{<r a} \cup \Sigma_{r}, \Lambda^{<r a} ; \mathbb{Z}\right)$.

(1) $\left(\sigma_{1}\right)^{* r}=0$ and $\left(\tau_{1}\right)^{* r}=0$.

(2) Some further products are described in the following tables:

\begin{tabular}{|c|c|c|}
\hline & $\left(\bar{\sigma}_{1}\right)^{*(r-1)} * \sigma_{1}$ & $\left(\bar{\sigma}_{1}\right)^{* r}$ \\
\hline \hline$\lambda_{r}=\lambda_{r}^{\min }$ and $\lambda_{n}=\lambda_{n}^{\min }$ & $\sigma_{r}$ & $\bar{\sigma}_{r}$ \\
\hline$\lambda_{r} \neq \lambda_{r}^{\min }$ & 0 & 0 \\
\hline
\end{tabular}

FIGURE 5. Homology level products

\begin{tabular}{|c|c|c|}
\hline & $\left(\bar{\tau}_{1}\right)^{\circledast(r-1)} \circledast \tau_{1}$ & $\left(\bar{\tau}_{1}\right)^{\circledast r}$ \\
\hline$\lambda_{r n}=\lambda_{r n}^{\max }$ & $\tau_{r}$ & $\bar{\tau}_{r}$ \\
\hline$\lambda_{r} \neq \lambda_{r}^{\max }$ & 0 & 0 \\
\hline
\end{tabular}

Figure 6. Cohomology level products

\footnotetext{
${ }^{3}$ If $M$ is orientable and $\gamma$ is prime then $\Gamma_{1}$ is orientable. This follows from the argument of $[\mathrm{R}] \S 2.2$, which is reproduced in the proof of Proposition 12.2 below. In addition, $\lambda_{1}$ and $\lambda_{2}$ have the same parity $\Longleftrightarrow$ all the negative bundles $\Gamma_{m}$ are orientable $\Longleftrightarrow$ all the $\lambda_{i}$ have the same parity, cf. $[\mathrm{R}, \mathrm{Wi}]$.
} 
(3) If $n-\lambda_{1}$ is even then $\left(\bar{\sigma}_{1}\right)^{* r}=0$.

(4) If $n-\lambda_{1}$ is odd then $\left(\bar{\tau}_{1}\right)^{\circledast r}=0$.

Proof. We begin with the parity statements (3) and (4). In general, if $\sigma \in H_{k}(\Lambda ; \mathbb{Z})$ and $\tau \in H^{k}(\Lambda ; \mathbb{Z})$ then $2 \sigma * \sigma=0$ if $n-k$ is odd and $2 \tau \circledast \tau=0$ if $n-k$ is even. This follows from Propositions 5.3 and 8.3 , and it implies the vanishing of $\left(\bar{\sigma}_{1}\right)^{* r}$ and $\left(\bar{\tau}_{1}\right)^{\circledast r}\left(n-\lambda_{1}\right.$ even, odd, respectively).

Statement (1) follows from the fact that the homology class $\sigma_{1}$ is supported on a closed subset $A \subset \Lambda^{\leq a}$ such that $A \cap \Sigma_{1}$ consists of a single point. By Proposition 5.4 the product $\sigma_{1} * \sigma_{1}$ is supported on the set $A * A^{\prime}$ where $A^{\prime} \subset \Lambda$ is a support set for $\sigma_{1}$ that intersects $\Sigma_{1}$ in a different point. Consequently $A * A^{\prime} \subset \Lambda^{<2 a}$. Similarly, the cohomology class $\tau_{1} \in$ $H^{\lambda}\left(\Lambda^{\leq a+\epsilon}, \Lambda^{<a}\right)$ is supported on a closed set $B \subset \Lambda^{\geq a}$ that intersects $\Sigma_{1}$ in a single point. The zeroes in the second row of each of the tables are also easily explained. The (level) homology classes $\left(\bar{\sigma}_{1}\right)^{* r}$ and $\bar{\sigma}_{r}$ have the same degree if and only if $\lambda_{r}=\lambda_{r}^{\min }$. The (level) cohomology classes $\left(\bar{\tau}_{1}\right)^{\circledast r}$ and $\bar{\tau}_{r}$ have the same degree iff $\lambda_{r}=\lambda_{r}^{\max }$. But all the $\lambda_{i}$ have the same parity, so if $\lambda_{r}$ does not attain its maximum or minimum value (i.e. if $\lambda_{r} \notin\left\{\lambda_{r}^{\min }, \lambda_{r}^{\max }\right\}$ ) then

$$
\lambda_{r}^{\min }+2 \leq \lambda_{r} \leq \lambda_{r}^{\max }-2 .
$$

In this case it follows from (11.1.1) that $\left(\bar{\sigma}_{1}\right)^{* r}=0$ and $\left(\bar{\tau}_{1}\right)^{\circledast r}=0$. The other calculations are similar. The remaining statements in Theorem 11.3 will be proven in the next two sections.

11.4. Case of maximal growth. In this section we assume $\lambda_{i}=\lambda_{i}^{\max }$ for $i \leq r n$. Choose $x_{0}=\gamma(0)$ for the base point of $M$. By Lemma 6.4 the index $\lambda_{r}$ equals the index $\lambda_{r}^{\Omega}$ of $\gamma^{r}$ in the based loop space $\Omega=\Omega_{x_{0}}$, and it coincides with the index of $\gamma^{r}$ in the spaces $T_{\gamma^{r}}^{\perp} \Lambda$ and $T_{\gamma^{r}}^{\perp} \Omega$ of vector fields $V(t)$ along $\gamma^{r}$ such that $V(t) \perp \gamma^{\prime}(t)$ for all $t$.

Let $W_{1}$ be a maximal negative subspace of $T_{\gamma}^{\perp} \Omega$ (so $\operatorname{dim}\left(W_{1}\right)=\lambda_{1}=\lambda_{1}^{\Omega}$ ). Let $W_{1}^{\bullet r}$ be the $r \lambda_{1}$-dimensional negative subspace of $T_{\gamma^{r}} \Omega$ consisting of concatenations $V_{1} \bullet V_{2} \bullet \cdots \bullet V_{r}$ of vector fields $V_{i} \in W_{1}$. Then $W_{1}^{\bullet r}$ is a maximal negative subspace of the kernel of

$$
\begin{aligned}
\nu: T_{\gamma^{r}}^{\perp} \Omega & \rightarrow T_{\gamma(0)}^{\perp} M \times \cdots \times T_{\gamma(0)}^{\perp} M \\
V & \mapsto\left(V\left(\frac{1}{r}\right), \cdots, V\left(\frac{r-1}{r}\right)\right)
\end{aligned}
$$

Choose a maximal negative subspace $W_{r} \subset T_{\gamma^{r}}^{\perp} \Omega$ containing $W_{1}^{\bullet r}$. Then

$$
\operatorname{dim}\left(W_{r}\right)=\lambda_{r}^{\Omega}=\lambda_{r}=\lambda_{r}^{\max }=r \lambda_{1}+(r-1)(n-1) .
$$

It follows that the restriction of $\nu$ to $W_{r}$ is surjective because its kernel has dimension $r \lambda_{1}$.

Let $\mathcal{C}_{r}$ be the $r$-leafed clover consisting of loops $\eta \in \Lambda$ such that $\eta(0)=\eta(i / r)$ for $i=$ $0,1,2, \cdots, r$. The exponential map $T_{\gamma^{r}}(\Lambda) \rightarrow \Lambda$ takes $W_{r}$ to a relative cycle in $\left(\Lambda^{\leq r a}, \Lambda^{\leq r a-\epsilon}\right)$ which we also denote by $W_{r}$, whose (relative) homology class is $\left[W_{r}\right]=\sigma_{r}$ (and $\left[W_{1}\right]=\sigma_{1}$ ). 
The function $J: \Lambda \times[0,1] \rightarrow \Lambda$ extends in an obvious way to a family of reparametrizations,

$$
J: W_{r} \times\left[\frac{1}{r}-\epsilon, \frac{1}{r}+\epsilon\right] \times\left[\frac{2}{r}-\epsilon, \frac{2}{r}+\epsilon\right] \times \cdots \times\left[\frac{r-1}{r}-\epsilon, \frac{r-1}{r}+\epsilon\right]
$$

which is transverse 4 to $C_{r}$ and such that

$$
J\left(W_{r} \times\left[\frac{1}{r}-\epsilon, \frac{1}{r}+\epsilon\right] \cdots \times\left[\frac{r-1}{r}-\epsilon, \frac{r-1}{r}+\epsilon\right]\right) \cap \mathcal{C}_{r}=W_{1} \bullet W_{1} \bullet \ldots \bullet W_{1}
$$

(Pontrjagin product). By (a relative version of) Proposition 8.5 we conclude that

$$
\begin{aligned}
\left\langle\bar{\tau}_{1} \circledast \bar{\tau}_{1} \circledast \ldots \circledast \bar{\tau}_{1}, \sigma_{r}\right\rangle & =\left\langle\bar{\tau}_{1} \circledast \bar{\tau}_{1} \circledast \ldots \circledast \bar{\tau}_{1},\left[W_{r}\right]\right\rangle \\
& =\left\langle\bar{\tau}_{1},\left[W_{1}\right]\right\rangle \cdot\left\langle\bar{\tau}_{1},\left[W_{1}\right]\right\rangle \cdot \ldots \cdot\left\langle\bar{\tau}_{1},\left[W_{1}\right]\right\rangle=1 .
\end{aligned}
$$

It follows that $\bar{\tau}_{1}^{\circledast r}=\overline{\tau_{r}}$. The calculation for $\bar{\tau}_{1}^{\circledast(r-1)} \circledast \tau_{1}$ is similar. The same technique, by explicitly displaying cycles, may be used to prove Theorem 13.2 below.

11.5. Case of minimal index growth. If $\left(D \Gamma_{r}, S \Gamma_{r}\right)$ denote the $\epsilon$-disk and sphere bundle of the negative bundle $\Gamma_{r} \rightarrow \Sigma_{r}$ then, for sufficiently small $\epsilon>0$ the exponential mapping $\exp : D \Gamma_{r} \rightarrow \Lambda$ is a smooth embedding whose image

$$
\left(\Sigma_{r}^{-}, \partial \Sigma_{r}^{-}\right)=\left(\exp \left(D \Gamma_{r}\right), \exp \left(S \Gamma_{r}\right)\right)
$$

is a smoothly embedded submanifold with boundary in $\Lambda$ that "hangs down" from the critical set $\Sigma_{r}$. Its dimension is $\lambda_{r}+1$ and its fundamental class is

$$
\bar{\sigma}_{r}=\left[\Sigma_{r}^{-}, \partial \Sigma_{r}^{-}\right] \in H_{\lambda_{r}+1}\left(\Lambda^{<r \ell} \cup \Sigma_{r}, \Lambda^{<r \ell}\right) \cong \mathbb{Z}
$$

where $\ell$ denotes the length of $\gamma$.

Now assume that $\lambda_{n}=\lambda_{n}^{\mathrm{min}}$. By Lemma 6.4 this implies that the difference between $\lambda_{1}^{\Omega}$ and $\lambda_{1}$ is the maximum possible: $\lambda_{1}=\lambda_{1}^{\Omega}+n-1$. Let $W_{1} \subset T_{\gamma}^{\perp} \Lambda$ be a maximal negative subspace. Then the mapping

$$
\nu: W_{1} \rightarrow T_{\gamma(0)}^{\perp} M, \quad \nu(V)=V(0)
$$

is surjective. Consequently

$$
\mathbf{e v}_{0}: \Sigma_{1}^{-} \rightarrow M
$$

is a submersion in a neighborhood of the closed geodesic $\gamma$. It follows that $\Sigma_{r}^{-}$and $\Sigma_{1}^{-}$are transverse over $M$ (in some neighborhood of $\gamma^{r}$ and $\gamma$ ) and

$$
\Sigma_{r}^{-} \times_{M} \Sigma_{1}^{-} \underset{\phi_{\min }}{\cong} \Sigma_{r}^{-} * \Sigma_{1}^{-}
$$

is a smooth submanifold of $\Lambda$ in a neighborhood of

$$
\Sigma_{r} \times_{M} \Sigma_{1} \underset{\phi_{\min }}{\cong} \Sigma_{r} * \Sigma_{1}=\Sigma_{r+1}
$$

\footnotetext{
${ }^{4}$ The restriction $J \mid W_{r}$ is transverse to $\mathcal{C}_{r}$ in the directions normal to $\gamma^{\prime}(0)$ because $\nu \mid W_{r}$ is surjective. The intervals $\left[\frac{i-1}{r}, \frac{i}{r}\right]$ take care of the tangential directions.
} 
and it is contained in $\Sigma_{r+1} \cup \Lambda^{<(r+1) \ell}$. Now assume the index growth is minimal up to level $r$, that is, $\lambda_{r}=r \lambda_{1}-(r-1)(n-1)$, so that

$$
\operatorname{dim}\left(\Sigma_{r}^{-} \times_{M} \Sigma_{1}^{-}\right)=\operatorname{dim}\left(\Sigma_{r+1}^{-}\right) .
$$

Then we may apply 5 Theorem C.2 to the embeddings

$$
\Sigma_{r+1} \subset \Sigma_{r}^{-} \times_{M} \Sigma_{1}^{-} \underset{\phi_{\min }}{\longrightarrow} \Lambda \leq(r+1) \ell
$$

to conclude that

$$
\left[\Sigma_{r}^{-} * \Sigma_{1}^{-}, \partial\left(\Sigma_{r}^{-} * \Sigma_{1}^{-}\right)\right]=\left[\Sigma_{r+1}^{-}, \partial \Sigma_{r+1}^{-}\right] \in H_{\lambda_{r+1}+1}\left(\Sigma_{r+1} \cup \Lambda^{<(r+1) \ell}, \Lambda^{<(r+1) \ell}\right) .
$$

(In fact we even obtain a local diffeomorphism $\tau: \Sigma_{r+1}^{-} \rightarrow \Sigma_{r}^{-} * \Sigma_{1}^{-}$between the negative submanifolds, by equation (C.3.1).) Using Proposition 5.6 we conclude that

$$
\left[\Sigma_{r}^{-}, \partial \Sigma_{r}^{-}\right] *\left[\Sigma_{1}^{-}, \partial \Sigma_{1}^{-}\right]=\left[\Sigma_{r+1}^{-}, \partial \Sigma_{r+1}^{-}\right]
$$

and, by induction, that

$$
\bar{\sigma}_{r+1}=\bar{\sigma}_{r} * \bar{\sigma}_{1}=\left(\bar{\sigma}_{1}\right)^{* r} * \bar{\sigma}_{1}=\left(\bar{\sigma}_{1}\right)^{*(r+1)}
$$

as claimed. The geometric calculation of the product $\overline{\sigma_{r}} * \sigma_{1}$ is similar. A similar procedure will be used to prove Theorem 12.4 below.

11.6. The non-nilpotent case. The case of isolated closed geodesics with slowest possible index growth was studied in [Hi2]; fastest possible index growth was studied in [Hi1] in a slightly different language because the $*$ and $\circledast$ products were not available at the time. The Chas-Sullivan homology product is modeled in the local geometry of an isolated closed geodesic with the slowest possible growth rate. The symmetry between the geometry in the case of slowest possible index growth (non-nilpotent level homology) and that of fastest possible index growth (non-nilpotent level cohomology) inspired the search for the cohomology product. We give statements here of two theorems on non-nilpotent products that are restatements of the "complementary theorem" (p. 3100 of [Hi1]) and the theorem (p. 3099 of [Hi1]):

11.7. Theorem. Let $\gamma$ be an isolated closed geodesic with non-nilpotent level homology. Let $L=$ length $(\gamma)$. Then, for any $\epsilon>0$, if $m \in \mathbb{Z}$ is sufficiently large there is a closed geodesic with length in the open internval $(m L, m L+\epsilon)$. It follows that $M$ has infinitely many closed geodesics.

11.8. Theorem. Let $\gamma$ be an isolated closed geodesic with non-nilpotent level cohomology. Let $L=$ length $(\gamma)$. Then, for any $\epsilon>0$, if $m \in \mathbb{Z}$ is sufficiently large there exists a closed geodesic with length in the open interval $(m L-\epsilon, m L)$. It follows that $M$ has infinitely many closed geodesics.

\footnotetext{
${ }^{5}$ The condition on the eigenvalues of the second derivative (in the hypotheses of Theorem C.2) is satisfied by the energy functional, as a consequence of Theorem 2.4.2 in $[\mathrm{K}]$.
} 


\section{Homology product when all geodesics are closed}

12.1. In this section $M$ denotes a compact $n$ dimensional Riemannian manifold. The coefficient group $G$ for homology is taken to be $G=\mathbb{Z}$ if $M$ is orientable, and $G=\mathbb{Z} /(2)$ otherwise. Throughout this section we assume that all geodesics $\gamma$ are closed and simply periodic with the same prime length $\ell$, meaning that: $\gamma(0)=\gamma(1), \gamma^{\prime}(0)=\gamma^{\prime}(1), \gamma$ is injective on $(0,1)$, and $L(\gamma)=\ell$ if $\gamma$ is prime.

For $r \geq 1$ denote by $\Sigma_{r} \subset \Lambda$ the critical set consisting of $r$-fold iterates of prime closed geodesics. There is a diffeomorphism $S M \cong \Sigma_{r}$ between the unit sphere bundle of $M$ and $\Sigma_{r}$, which assigns to each unit tangent vector $v$ the $r$-fold iterate of the prime geodesic with initial condition $v$. It follows that the nullity of each geodesic is at least $\operatorname{dim}\left(\Sigma_{r}\right)-1=2 n-2$. Since this is the maximum nullity possible, we see that the nullity $\nu_{r}$ of every closed geodesic is $2 n-2$. In particular, each $\Sigma_{r}$ is a nondegenerate critical submanifold (in the sense of Bott), with critical value $F\left(\Sigma_{r}\right)=r \ell$, and this accounts for all the critical points of the Morse function $F=\sqrt{E}$. Moreover, for any $c \in \mathbb{R}$, the singular and Cech homology $H_{*}\left(\Lambda^{\leq c}\right)$ agree, by Proposition 3.3. Every geodesic $\gamma \in \Sigma_{r}$ has the same index, ([Be] Thm. 7.23) say, $\lambda_{r}$. By Proposition (6.1.1),$\lambda_{r} \leq r \lambda_{1}+(r-1)(n-1)$. By (6.1.2), $\lambda_{r} \geq r \lambda_{1}+(r-1)(n-1)$, hence the index growth is maximal,

$$
\lambda_{r}=\lambda_{r}^{\max }=r \lambda_{1}+(r-1)(n-1) .
$$

As in Theorem C.2, let $\Gamma_{r} \rightarrow \Sigma_{r}$ be the negative definite bundle. It is a real vector bundle whose rank is $\lambda_{r}$.

12.2. Proposition. If $M$ is orientable (and all geodesics on $M$ are closed with the same prime period) then for any $r$ the negative bundle $\Gamma_{r}$ is also orientable.

Proof. Fix $r$ and let $\gamma_{0} \in \Sigma_{r}$ be a basepoint. Set $x_{0}=\gamma_{0}(0) \in M$. Using the long exact sequence for the fibration $S M \rightarrow M$ we see that the projection $\Sigma_{r} \rightarrow M$ induces an isomorphism $\pi_{1}\left(\Sigma_{r}, \gamma_{0}\right) \cong \pi_{1}\left(M, x_{0}\right)$. If $\lambda_{1}>0$ then by $\mathrm{Be}$ Thm. 7.23 the manifold $M$ is simply connected, so if $\operatorname{dim}(M) \geq 3$ the same is true of $\Sigma_{r}$, hence every vector bundle on $\Sigma_{r}$ is orientable. If $\operatorname{dim}(M)=2$ then $M=S^{2}$ is the 2-sphere and $\Gamma_{r}$ is orientable by inspection.

So we may assume that $\lambda_{1}=0$. By [Be] Thm 7.23 this implies that $M$ is diffeomorphic to real projective space and $\pi_{1}\left(M, x_{0}\right) \cong \mathbb{Z} /(2)$. Since $M$ is orientable, $n=\operatorname{dim}(M)$ is odd.

The bundle $\Gamma_{r} \rightarrow \Sigma_{r}$ is orientable iff its restriction to each loop in $\Sigma_{r}$ is orientable, and it suffices to check this on any loop in the single non-trivial class in $\pi_{1}\left(\Sigma_{r}, \gamma_{0}\right)$. We may even take that loop to be the canonical lift $\widetilde{\gamma}:[0,1] \rightarrow S M$,

$$
\widetilde{\gamma}(t)=\left(\gamma(t), \gamma^{\prime}(t) /\left\|\gamma^{\prime}(t)\right\|\right)
$$

of a periodic prime geodesic $\gamma:[0,1] \rightarrow M$ with $\gamma(0)=x_{0}$. (Since each geodesic is determined by its initial conditions, it follows that $\gamma_{0}=\gamma^{r}$. This geodesic loop is contractible in $M$ iff $r$ is even.) 
Following [R] $\S 2.2$, the $S^{1}$ action on $\Gamma_{r} \rightarrow \Sigma_{r}$ corresponds to an operator $T: E \rightarrow E$ such that $T^{r}=I$, where $E=\Gamma_{r, \gamma_{0}}$ is the fiber of $\Gamma_{r}$ at the basepoint $\gamma_{0}$. Then $\operatorname{det}(T)= \pm 1$ and the bundle $\Gamma_{r} \mid \widetilde{\gamma}$ is orientable iff $\operatorname{det}(T)=+1$. If $\lambda$ is an eigenvalue of $T$ then $\lambda^{r}=1$. Those eigenvalues which are not equal to \pm 1 come in complex conjugate pairs. Hence, $\operatorname{det}(T) \neq 1$ iff the dimension of the -1-eigenspace of $T$ is odd. If $r$ is odd then -1 is never an eigenvalue of $T$ so $\operatorname{det}(T)=1$. Thus we may assume that $r$ is even. As remarked in $[\mathrm{R}]$, by $[\mathrm{K}] \S 3.2 .9$, $\S 4.1 .5$ (see pp. 128,129), since $r$ is even, the dimension of the -1 -eigenspace of $T$ is equal to the quantity $I_{\gamma}(-1)$ of $[\mathrm{R}](\S 1.1)$ and $[\mathrm{K}](\S 4.1)$, namely

$$
I_{\gamma}(-1)=\lambda_{2}-\lambda_{1}=n-1
$$

which is even. Therefore $\Gamma_{r} \mid \widetilde{\gamma}$ is orientable, so the bundle $\Gamma_{r} \rightarrow \Sigma_{r}$ is orientable.

It follows from Theorem C.2 that a choice of orientation for $\Gamma_{r}$ determines an isomorphism

$$
h_{r}: H_{i}\left(\Sigma_{r} ; G\right) \cong H_{i+\lambda_{r}}\left(\Lambda^{\leq r \ell}, \Lambda^{<r \ell} ; G\right)
$$

where $G=\mathbb{Z}$ if $M$ is orientable and $G=\mathbb{Z} /(2)$ otherwise.

12.3. The non-nilpotent homology class. For any $c \in \mathbb{R}$, the long exact sequence for the pair $\left(\Lambda^{\leq c}, \Lambda_{0}\right)$ is canonically split by the evaluation mapping $\mathbf{e v}_{0}: \Lambda^{\leq c} \rightarrow \Lambda_{0}$ so for any Abelian group $G$ we obtain a canonical isomorphism

$$
H_{i}\left(\Lambda^{\leq c} ; G\right) \cong H_{i}\left(\Lambda_{0} ; G\right) \oplus H_{i}\left(\Lambda^{\leq c}, \Lambda_{0} ; G\right) .
$$

Taking $c=\ell=F\left(\Sigma_{1}\right)$ and using Theorem C.2 gives a canonical isomorphism

$$
H_{i}\left(\Lambda^{\leq \ell} ; G\right) \cong H_{i}\left(\Lambda_{0} ; G\right) \oplus H_{i-\lambda}\left(\Sigma_{1} ; G\right) .
$$

where $G=\mathbb{Z}$ if $M$ is orientable, and $G=\mathbb{Z} /(2)$ otherwise. The manifold $\Sigma_{1}$ is orientable (whether or not $M$ is), since $T \Sigma_{1} \oplus \mathbf{1} \cong h^{*}(T M) \oplus h^{*}(T M)$. Choose an orientation of $\Sigma_{1}$ with a resulting fundamental class $\left[\Sigma_{1}\right] \in H_{2 n-1}\left(\Sigma_{1} ; G\right)$. Define

$$
\Theta \in H_{2 n-1+\lambda_{1}}(\Lambda \leq \ell)
$$

to be its image under the isomorphism (12.3.2). Set $b=\lambda_{1}+n-1$.

12.4. Theorem. Let $M$ be an $n$ dimensional compact Riemannian manifold, all of whose geodesics are simply periodic with the same prime length $\ell$. If $M$ is orientable let $G=\mathbb{Z}$, otherwise set $G=\mathbb{Z} /(2)$. Then the following statements hold.

(1) the energy $E: M \rightarrow \mathbb{R}$ is a perfect Morse-Bott function for $H_{*}(\Lambda ; G)$, that is, for each $r \geq 1$ every connecting homomorphism vanishes in the long exact sequence

$$
\longrightarrow H_{i}\left(\Lambda^{<r \ell} ; G\right) \longrightarrow H_{i}\left(\Lambda^{\leq r \ell} ; G\right) \longrightarrow H_{i}\left(\Lambda^{\leq r \ell}, \Lambda^{<r \ell} ; G\right) \longrightarrow
$$

(2) the product $* \Theta: H_{i}\left(\Lambda, \Lambda_{0} ; G\right) \rightarrow H_{i+b}\left(\Lambda, \Lambda_{0} ; G\right)$ with the class $\Theta$ is injective, and

(3) for all $r \geq 1$ this product induces an isomorphism on level homology,

$$
w_{r}: H_{i}\left(\Lambda^{\leq r \ell}, \Lambda^{<r \ell} ; G\right) \rightarrow H_{i+b}\left(\Lambda^{\leq(r+1) \ell}, \Lambda^{<(r+1) \ell} ; G\right) .
$$


Proof. Assume, by induction on $r$ that $\beta_{r}: H_{i}\left(\Lambda^{\leq r \ell}, \Lambda_{0} ; G\right) \rightarrow H_{i}\left(\Lambda^{\leq r \ell}, \Lambda^{<r \ell} ; G\right)$ is surjective for all $i$. The case $r=1$ is handled by equation (12.3.2). Consider the following commutative diagram, where the vertical mappings are given by the Chas-Sullivan product $* \Theta$,

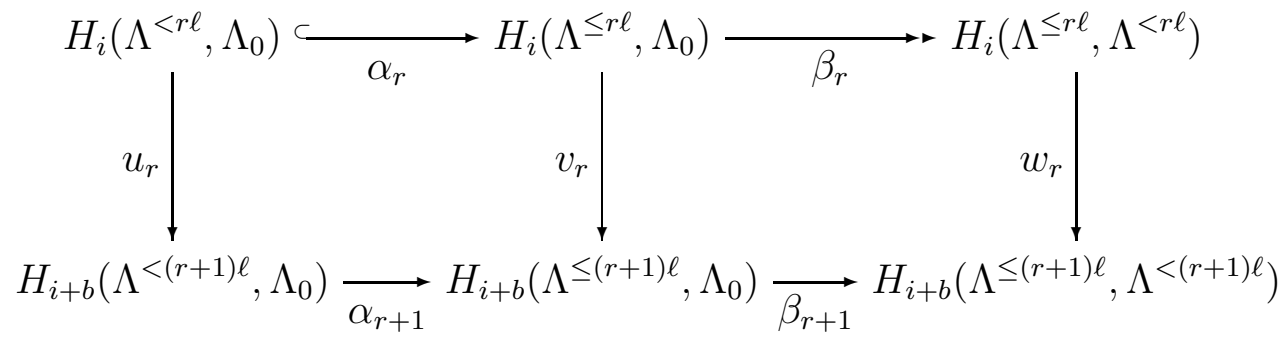

We will show below that the mapping $w_{r}$ is an isomorphism. Assuming this for the moment, it follows that $\beta_{r+1}$ is surjective in all degrees. Hence the horizontal sequences in this diagram split into short exact sequences (so the Morse function is perfect). Therefore $u_{r}$ is injective if and only if $v_{r}$ is injective. However $v_{r}$ may be identified with the mapping $u_{r+1}$ under the isomorphism $H_{i}\left(\Lambda^{\leq r \ell}\right) \cong H_{i}\left(\Lambda^{<(r+1) \ell}\right)$ so it is injective by induction. (The mapping $u_{1}$ is trivially injective.) The rest of $\$ 12$ will be devoted to proving that $w_{r}$ is an isomorphism.

12.5. Theorem. After composing with the isomorphism

$$
h_{r}: H_{*}(S M) \rightarrow H_{*}\left(\Lambda^{\leq r \ell}, \Lambda^{<r \ell}\right)
$$

(where $S M$ denotes the unit sphere bundle of the tangent bundle to $M$ ), the Chas-Sullivan product becomes the intersection product on homology, which is to say that the following diagram commutes:

$$
\begin{gathered}
H_{i}\left(\Lambda^{\leq r \ell}, \Lambda^{<r \ell}\right) \times H_{j}\left(\Lambda^{\leq \ell}, \Lambda^{<\ell}\right) \stackrel{*}{\longrightarrow} H_{i+j-n}\left(\Lambda^{\leq(r+1) \ell}, \Lambda^{<(r+1) \ell}\right) \\
h_{r} \times h_{1}\left|\cong \quad h_{r+1}\right| \cong \\
H_{i-\lambda_{r}}(S M) \times H_{j-\lambda_{1}}(S M) \longrightarrow H_{i-\lambda_{r}+j-\lambda_{1}-2 n+1}(S M)
\end{gathered}
$$

where the bottom row denotes the intersection product in homology

We remark that this immediately implies that $w_{r}$ is an isomorphism because the mapping $w_{r}$ is the C-S product with the unique top dimensional class in $H_{*}\left(\Lambda^{\leq \ell}, \Lambda^{<\ell}\right)$ which becomes the fundamental class $[S M] \in H_{2 n-1}(S M)$ under the vertical isomorphism in the above diagram. But the intersection with the fundamental class is the identity mapping $H_{*}(S M) \rightarrow$ $H_{*}(S M)$.

Proof. The set $\Sigma_{r} * \Sigma_{1}=\phi_{\frac{r}{r+1}}\left(\Sigma_{r} \times_{M} \Sigma_{1}\right)$ consists of pairs of composable loops; the first is an $r$-fold iterate of a prime geodesic and the second is a single prime geodesic; all parametrized proportionally with respect to arclength. This set contains $\Sigma_{r+1}$ as a submanifold of codimension $n-1$. In fact the inclusion

$$
\Sigma_{r+1} \rightarrow \Sigma_{r} * \Sigma_{1} \rightarrow \Sigma_{r} \times \Sigma_{1}
$$


is the diagonal mapping $S M \rightarrow S M \times S M$.

Let $\left(\Sigma_{r}^{-}, \partial \Sigma_{r}^{-}\right)=\left(\exp \left(D \Gamma_{r}\right), \exp \left(\partial D \Gamma_{r}\right)\right)$ be the negative submanifold that hangs down from $\Sigma_{r}$, as in Proposition C.2. where $D \Gamma_{r}$ denotes a sufficiently small disk bundle in the negative bundle $\Gamma_{r} \rightarrow \Sigma_{r}$ and where $\partial D \Gamma_{r}$ denotes its bounding sphere bundle. Then $\operatorname{dim}\left(\Sigma_{r}^{-} \times_{M} \Sigma_{1}^{-}\right)=\operatorname{dim}\left(\Sigma_{r+1}^{-}\right)$so we can apply Theorem C.2 to the embeddings

$$
\Sigma_{r+1} \subset \Sigma_{r}^{-} \times_{M} \Sigma_{1}^{-} \underset{\phi_{\min }}{\longrightarrow} \Lambda \leq(r+1) \ell
$$

followed by an arbitrarily brief flow under the vector field $-\nabla F$. The condition on the eigenvalues of the second derivative (in the hypotheses of Theorem C.2) is satisfied by the energy functional, as a consequence of Theorem 2.4.2 in [K]. As in (C.3.1) we obtain a local (in a neighborhood of $\Sigma_{r+1}$ ) diffeomorphism

$$
\tau: \Sigma_{r+1}^{-} \rightarrow \Sigma_{r}^{-} \times_{M} \Sigma_{1}^{-}
$$

between the negative submanifolds, see Figure 7. By Proposition 5.6 the Chas-Sullivan product is given by the composition down the right side of this figure.

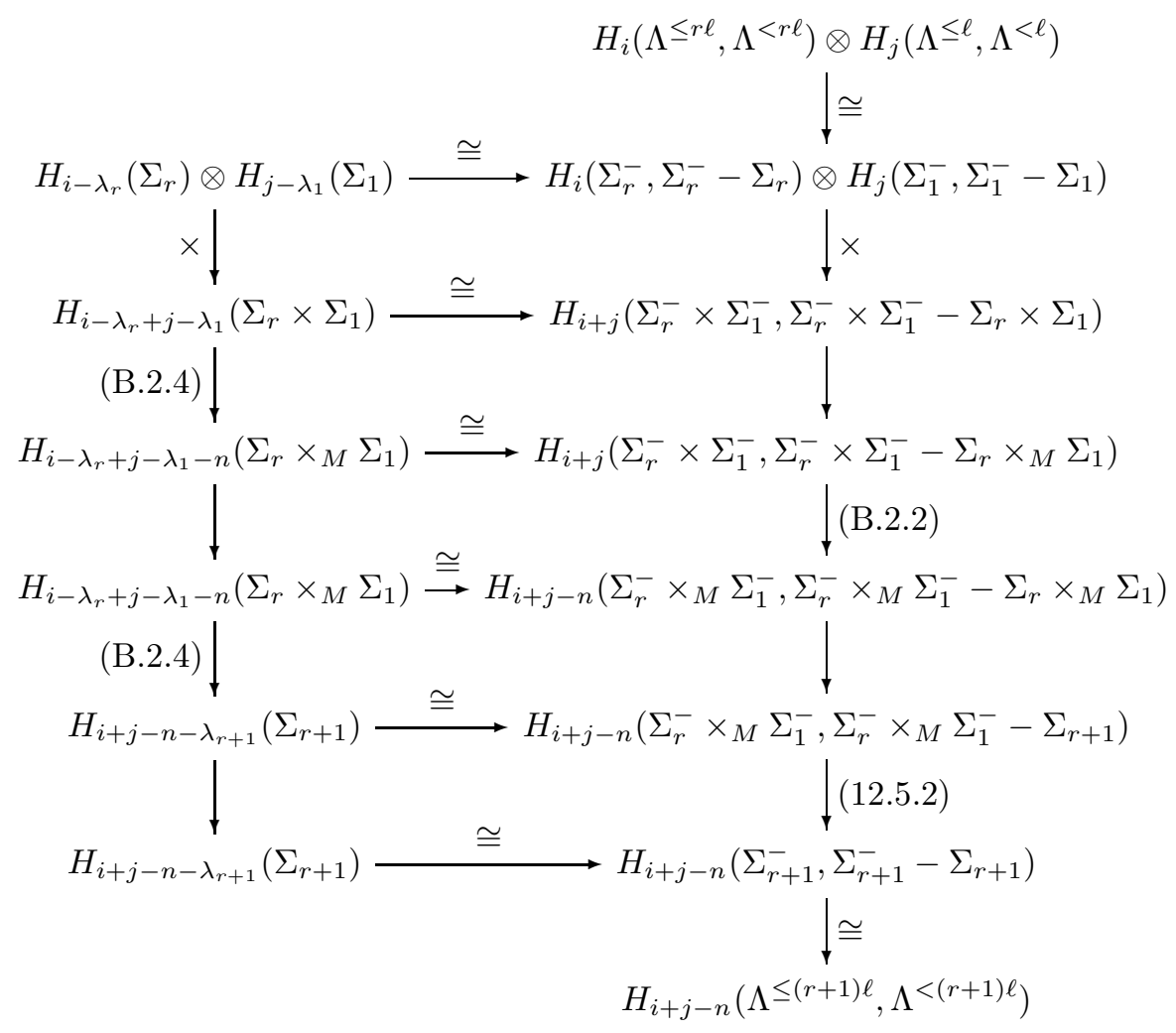

Figure 7. The C-S product when all geodesics are closed

On the other hand, the composition down the left side of the diagram is the intersection pairing because the composition down the middle four rows is just the Gysin pull back for 
the (diagonal) embedding (12.5.1). This completes the proof of Theorem 12.5 and hence

also of Theorem 12.4,

12.6. Define the filtration $0 \subset I_{0} \subset I_{1} \cdots \subset H_{*}\left(\Lambda, \Lambda_{0} ; G\right)$ by

$$
I_{r}=\text { Image }\left(H_{*}\left(\Lambda^{\leq r \ell}, \Lambda_{0} ; G\right) \rightarrow H_{*}\left(\Lambda, \Lambda_{0} ; G\right)\right) .
$$

By Proposition [5.4, $I_{r} * I_{s} \subset I_{r+s}$ so the Chas-Sullivan product induces a product on the associated graded group,

$$
\operatorname{Gr}_{I} H_{*}\left(\Lambda, \Lambda_{0} ; G\right)=\bigoplus_{r=1}^{\infty} I^{r} / I^{r-1} \cong \bigoplus_{r=1}^{\infty} H_{*}\left(\Lambda^{\leq r \ell}, \Lambda^{<r \ell}\right)
$$

which therefore coincides with the level homology ring (5.4.4). Let $H_{*}(S M ; G)$ be the homology (intersection) ring of the unit sphere bundle and let $H_{*}(S M)[T]_{\geq 1}=T H_{*}(S M)[T]$ be the ideal of polynomials of degree $\geq 1$.

12.7. Corollary. The mapping

$$
\begin{gathered}
\Phi: H_{*}(S M ; G)[T]_{\geq 1} \rightarrow G r_{I} H_{*}\left(\Lambda, \Lambda_{0} ; G\right) \\
\Phi\left(a T^{m}\right)=h_{1}(a) * \Theta^{*(m-1)} \in H_{\operatorname{deg}(a)+\lambda_{1}+(m-1) b}\left(\Lambda^{\leq(m) \ell}, \Lambda^{<(m) \ell} ; G\right)
\end{gathered}
$$

is an isomorphism of rings.

Proof. This follows immediately from Theorems 12.4 and 12.5 .

\section{Cohomology products when all geodesics are closed}

13.1. As in $\$ 12.1$, assume that $M$ is compact $n$ dimensional and all geodesics on $M$ are simply periodic with the same prime length, $\ell$. Let $\Sigma_{r} \subset \Lambda$ denote the submanifold consisting of the $r$-fold iterates of prime geodesics. It is a nondegenerate critical submanifold, diffeomorphic to the unit sphere bundle $S M$, having index $\lambda_{r}=r \lambda_{1}+(r-1)(n-1)$ and critical value $F\left(\Sigma_{r}\right)=r a$. Let $D \Gamma_{r}, S \Gamma_{r}$ be the unit disk bundle and unit sphere bundle of the negative bundle $\Gamma_{r} \rightarrow \Sigma_{r}$. If $M$ is orientable, take cohomology with coefficients in the ring $G=\mathbb{Z}$ and choose orientations of $M$ and $\Gamma_{r}$, otherwise take coefficients in in $G=\mathbb{Z} /(2)$. Theorem C.2 then gives an isomorphism

$$
h_{r}: H^{i}\left(\Sigma_{r}\right) \stackrel{\cong}{\longrightarrow} H^{i+\lambda_{r}}\left(\Lambda^{\leq r \ell}, \Lambda^{<r \ell}\right)
$$

by identifying each with $H^{i+\lambda_{r}}\left(D \Gamma_{r}, S \Gamma_{r}\right)$. Let $b=\lambda_{1}+n-1$. Define

$$
\Omega \in H^{\lambda_{1}}\left(\Lambda^{\leq \ell}, \Lambda_{0}\right) \cong H^{0}\left(\Sigma_{1}\right)
$$

to be the image $h_{1}(1)$ of the element 1.

13.2. Theorem. Assume $M$ satisfies the above hypotheses. Then 
(1) The energy $E: \Lambda \rightarrow \mathbb{R}$ is a perfect Morse function for cohomology, meaning that for each $r \geq 1$ the connecting homomorphism vanishes in the long exact sequence

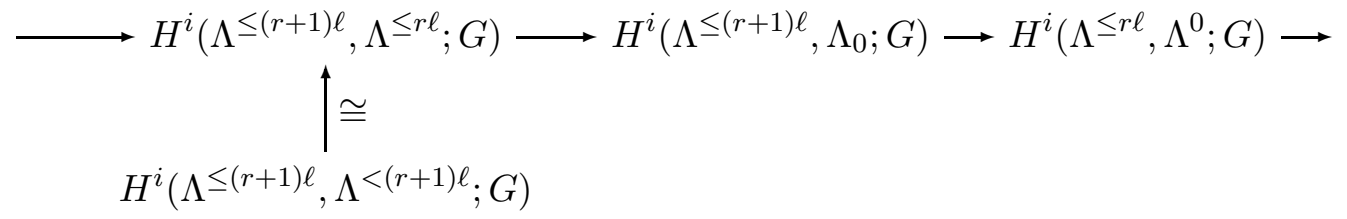

(2) The product $\circledast \Omega: H^{i}\left(\Lambda, \Lambda_{0}\right) \rightarrow H^{i+b}\left(\Lambda, \Lambda_{0}\right)$ is injective and

(3) this product induces an isomorphism

$$
w_{r}: H^{i}\left(\Lambda^{\leq r \ell}, \Lambda^{<r \ell}\right) \rightarrow H^{i+b}\left(\Lambda^{\leq(r+1) \ell}, \Lambda^{<(r+1) \ell}\right)
$$

for all $r \geq 1$ and all $i \geq 0$.

As in the proof of Theorem 12.4, part (3) implies parts (1) and (2). Part (3) follows from the stronger statement,

\subsection{Theorem. After composing with the isomorphism}

$$
h_{r}: H^{*}(S M) \rightarrow H^{*}\left(\Lambda^{\leq r \ell}, \Lambda^{<r \ell}\right)
$$

the cohomology product becomes the cup product on cohomology, which is to say that the following diagram commutes (recall that $\lambda_{r+1}=\lambda_{1}+\lambda_{r}+n-1$ ),

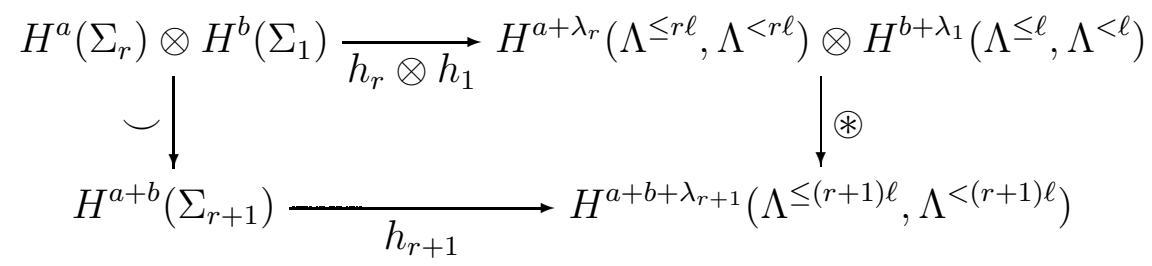

The proof appears in the next few sections. In order to use Proposition 8.6 we will need to work in the space $\mathcal{A}$ of PPAL loops.

13.4. Fix $r \geq 1$. Let $r \ell^{+}=r \ell+2 \epsilon, r \ell^{-}=r \ell-\epsilon, \ell^{+}=\ell+2 \epsilon$, and $\ell^{-}=\ell-\epsilon$. Set $j=a+b+\lambda_{r}+\lambda_{1}$ so that $j+n-1=a+b+\lambda_{r+1}$. It is convenient to replace the gluing map $\phi_{\frac{1}{2}}: \mathcal{A} \times_{M} \mathcal{A} \rightarrow \mathcal{A}_{\frac{1}{2}}$ with the topologically equivalent embedding $\phi_{\frac{r}{r+1}}: \mathcal{A} \times_{M} \mathcal{A} \rightarrow \mathcal{A}_{\frac{r}{r+1}}$, which approximates $\phi_{\min }$ near $\Sigma_{r} \times \Sigma_{1}$, in fact,

$$
\Sigma_{r} * \Sigma_{1}=\phi_{\min }\left(\Sigma_{r} \times_{M} \Sigma_{1}\right)=\phi_{\frac{r}{r+1}}\left(\Sigma_{r} \times_{M} \Sigma_{1}\right) .
$$

We will write

$$
\mathcal{F}_{\frac{r}{r+1}}^{<a,<b} \text { for } \phi_{\frac{r}{r+1}}\left(\mathcal{F}_{\mathcal{A}}^{<a,<b}\right) .
$$

Similarly we replace mapping $J_{\mathcal{A}}: \mathcal{A} \times[0,1] \rightarrow \mathcal{A}_{\frac{1}{2}}$ with the mapping

$$
J_{r}: \mathcal{A} \times[0,1] \rightarrow \mathcal{A}_{\frac{r}{r+1}}
$$


given by $J_{r}(\alpha, s)=\alpha \circ \theta_{\frac{r}{r+1} \rightarrow s}$ where $A_{\frac{r}{r+1}}$ and $\theta_{\frac{r}{r+1} \rightarrow s}$ are defined by replacing $\frac{1}{2}$ with $\frac{r}{r+1}$ in 98.1. The mapping $\mathcal{A}^{\leq(r+1) \ell} \rightarrow \mathcal{A}_{\frac{r}{r+1}}^{\leq r \ell} \leq \ell$ given by $\alpha \mapsto J_{r}\left(\alpha, \frac{r}{r+1}\right)$ is a homotopy equivalence; its inverse assigns to a pair of joinable PPAL paths $\alpha, \beta \in \mathcal{A}_{\frac{r}{r+1}}$ (with $\alpha(1)=\beta(0)$ and $\beta(1)=\alpha(0))$ with lengths $\leq r \ell$ and $\leq \ell$ respectively, the path $\phi_{\min }(\alpha, \beta)$ obtained by joining them at time $L(\alpha) /(L(\alpha)+L(\beta))$.

Let $I^{\prime}$ be the closed interval $I^{\prime}=\left[\frac{r \ell-\epsilon}{(r+1) \ell+\epsilon}, \frac{r \ell+2 \epsilon}{(r+1) \ell+\epsilon}\right]$ as in 88.11 , Then

$$
J_{r}\left(\mathcal{A}^{<(r+1) \ell+\epsilon} \times I^{\prime}\right) \subset \mathcal{A}_{\frac{r}{r+1}}^{<\ell^{+},<\ell^{+}}
$$

and $J_{r}$ takes both $\mathcal{A}^{<(r+1) \ell+\epsilon} \times \partial I^{\prime}$ and $\mathcal{A}^{<(r+1) \ell-2 \epsilon} \times I^{\prime}$ into the subset

$$
\mathcal{A}_{\frac{r}{r+1}}^{<r \ell^{+},<\ell^{+}}-\phi_{\frac{r}{r+1}}\left(\mathcal{F}_{\mathcal{A}}^{\left[r \ell^{-}, r \ell^{+}\right),\left[\ell^{-}, \ell^{+}\right)}\right) .
$$

13.5. Recall from \$12.1 that the negative bundle $\Gamma_{r}$ over $\Sigma_{r}$ is orientable if $M$ is orientable, and that the exponential defines a diffeomorphism $e_{r}$ of a sufficiently small disk bundle and its bounding sphere bundle, $\left(D \Gamma_{r}, \partial D \Gamma_{r}\right)$ onto a submanifold with boundary, $\left(\Sigma_{r}^{-}, \partial \Sigma_{r}\right)$ in $\Lambda^{\leq r \ell}$, such that $e_{r}\left(D \Gamma_{r}-\Sigma_{r}\right) \subset \Lambda^{<r \ell}$ (where $\Sigma_{r}$ is the zero section). Using the homotopy equivalence $Q: \Lambda^{\leq r \ell} \rightarrow \mathcal{A}^{\leq r \ell}$ of Proposition 2.2, we may assume that $\Sigma_{r}^{-} \subset \mathcal{A}^{\leq r \ell}$ so we obtain isomorphisms which we also denote by

$$
h_{r}: H^{i}\left(\Sigma_{r}\right) \cong H^{i+\lambda_{r}}\left(\Sigma_{r}^{-}, \partial \Sigma_{r}^{-}\right) \cong H^{i+\lambda_{r}}\left(\mathcal{A}^{\leq r \ell}, \mathcal{A}^{<r \ell}\right) .
$$

Moreover, equation (12.5.2) gives a diffeomorphism $\tau: \Sigma_{r+1}^{-} \rightarrow \Sigma_{r}^{-} \times_{M} \Sigma_{1}^{-} \cong \Sigma_{r}^{-} * \Sigma_{1}^{-}$. The following diagram may help in sorting out these different spaces.

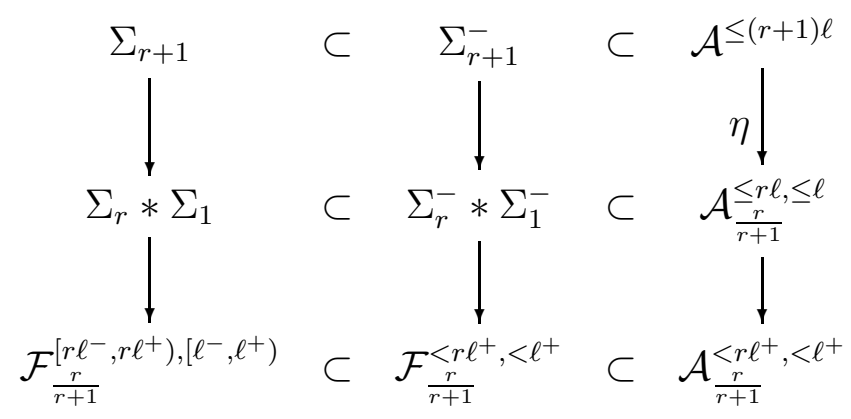

In order to compact the notation, for the rest of the section we will write

$$
H^{*}(Y, \sim A) \text { for } H^{*}(Y, Y-A) \text {. }
$$

13.6. We are now in a position to expand the diagram in Theorem 13.3, This is accomplished in Figure 8, Here, $j=a+b+\lambda_{r}+\lambda_{1}$ so that $j+n-1=a+b+\lambda_{r+1}$. Each of the rectangles in this diagram is obviously commutative except possibly for the portion denoted 1 , which we now explain, as it involves the somewhat mysterious degree shift of 1 , and its relationship to the mapping $J_{r}$. 


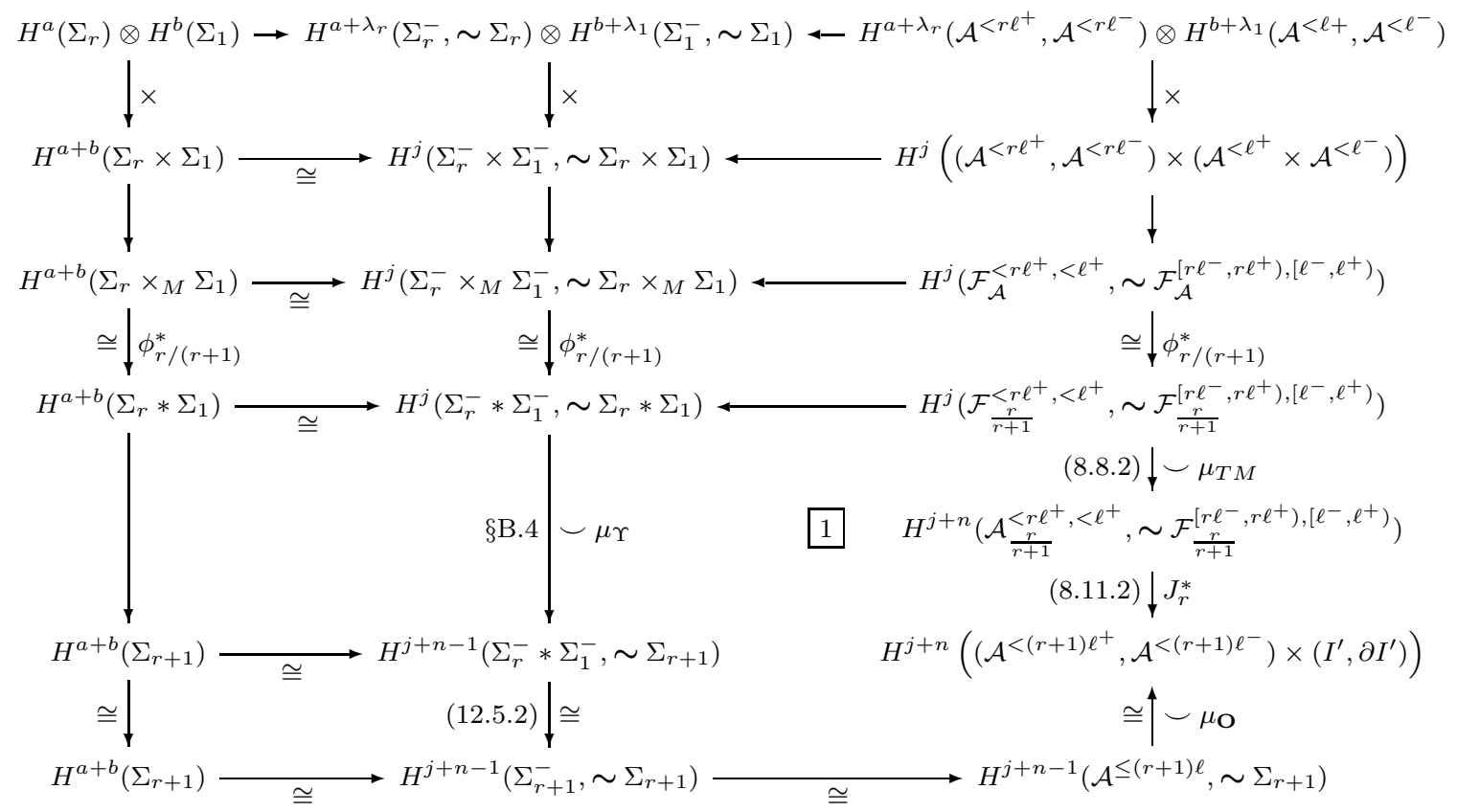

Figure $8 . \circledast$ product

The figure eight space $\mathcal{F}$ has a normal bundle in $\Lambda$ that is isomorphic to the (pullback of the) normal bundle of the diagonal in $M \times M$, and hence to the tangent bundle $T M$ of $M$. Its Thom class is denoted $\mu_{T M}$ and the Thom isomorphism (8.8.2) is given by the cup product with this Thom class. The normal bundle of $\Sigma_{r+1}$ in $\Sigma_{r} \times_{M} \Sigma_{1}$ is denoted $\Upsilon$. The Gysin mapping (labeled $\$$ B.4 in the diagram) is given by the cup product with the Thom class $\mu_{\Upsilon}$. The Künneth isomorphism at the lower right corner of the diagram is given by the cup product with the generator of $H^{1}\left(I^{\prime}, \partial I^{\prime}\right)$ which may be identified with the Thom class $\mu_{\mathbf{O}}$ of the trivial one dimensional bundle $\mathbf{O}$ on the interval $I^{\prime}$.

So to prove that 1 commutes we need to compare the Thom class $\mu_{T M}$ with the product of Thom classes $\mu_{\Upsilon} \cup \mu_{\mathbf{O}}$. It suffices to construct a vector bundle isomorphism $J_{r}^{*}(T M) \cong \Upsilon \oplus \mathbf{O}$.

The critical set $\Sigma_{r+1}$ is a submanifold of codimension $n-1$ in $\Sigma_{r} * \Sigma_{1}$. A point in the latter space is an $r$-fold iterate of a prime closed geodesic followed by a prime closed geodesic with the same base point, all parametrized proportionally with respect to arclength, so it is determined by a triple $(p, u, v)$ where $p \in M$ and $u, v \in S_{p}$ are unit tangent vectors at $p$. This point lies in $\Sigma_{r+1}$ if and only if $u=v$. It follows that the normal bundle $\Upsilon$ of $\Sigma_{r+1}$ in $\Sigma_{r} * \Sigma_{1}$ may be naturally identified with the bundle $\operatorname{ker}(d \pi)$ of tangents to the fibers of the projection $\pi: S M \rightarrow M$. But there is another way to view this bundle.

Let $\nu$ be the tautological (trivial) bundle over $S M$ whose fiber at the point $v \in S_{p}$ is the 1-dimensional span $\langle v\rangle \subset T_{p} M$. Then $\pi^{*}(T M) \cong \nu \oplus \nu^{\perp}$ where $\nu^{\perp}$ is the bundle whose fiber over $v \in S_{p}$ is $v^{\perp}$. For any $v \in S_{p}$ the inclusion of the unit tangent sphere $S_{p} \subset T_{p} M$ 
induces an injection $\operatorname{ker}(d \pi) \hookrightarrow T_{p} M$ whose image is $\nu^{\perp}$. In this way we obtain a canonical isomorphism $\Upsilon \cong \nu^{\perp}$ and therefore an isomorphism $\pi^{*}(T M) \cong \Upsilon \oplus \nu$.

Consider the restriction $J_{r}: \Sigma_{r+1} \times I^{\prime} \rightarrow \Lambda$, say $\beta=J_{r}(\alpha, s)$. Then $\frac{\partial J_{r}}{\partial s}(\alpha, s)$ is a vector field along $\beta$ that is a multiple of the tangent vector $\beta^{\prime}$ since the $s$ factor only changes the parametrization. This gives an isomorphism between $J_{r}^{*}(\nu)$ and the trivial 1-dimensional tangent bundle $T I^{\prime}$ on $\Sigma_{r+1} \times I^{\prime}$. In summary we have constructed an isomorphism $J_{r}^{*}(T M) \cong$ $\Upsilon \oplus \mathbf{O}$. This completes the proof that the diagram in Theorem 13.3 commutes, so it completes the proof of Theorem 13.2 .

13.7. Level cohomology ring. Continue with the assumption that all geodesics on $M$ are simply periodic with the same prime length $\ell$. Define the filtration $H^{*}\left(\Lambda, \Lambda_{0} ; G\right)=I^{0} \supset$ $I^{1} \supset \cdots$ by

$$
I^{r}=\text { Image }\left(H^{*}\left(\Lambda, \Lambda^{\leq r \ell} ; G\right) \rightarrow H^{*}\left(\Lambda, \Lambda_{0} ; G\right)\right) .
$$

Each $I^{r} \subset H^{*}\left(\Lambda, \Lambda_{0}\right)$ is an ideal (with respect to the $\circledast$ product) and $I^{r} \circledast I^{s} \subset I^{r+s}$. Since the Morse function is perfect it induces an isomorphism

$$
G r^{I} H^{*}\left(\Lambda, \Lambda_{0}\right) \cong \underset{r \geq 1}{\oplus} H^{*}\left(\Lambda^{\leq r \ell}, \Lambda^{<r \ell}\right)
$$

between the associated graded ring and the level cohomology ring. Let $H^{*}(S M ; G)$ denote the cohomology ring of the unit sphere bundle of $M$.

13.8. Corollary. The mapping (cf. \$12.6)

$$
\Psi: H^{*}(S M ; G)[T]_{\geq 1} \rightarrow G r^{I} H^{*}\left(\Lambda, \Lambda_{0} ; G\right)=\underset{r \geq 1}{\oplus} H_{*}\left(\Lambda^{\leq r \ell}, \Lambda^{<r \ell}\right)
$$

given by

$$
\Psi\left(a T^{m}\right)=h_{1}(a) \circledast \Omega^{\circledast(m-1)} \in H^{\operatorname{deg}(a)+\lambda_{1}+(m-1) b}\left(\Lambda^{\leq(m) \ell}, \Lambda^{<(m) \ell} ; G\right)
$$

is an isomorphism of rings.

Proof. Just as in Corollary 12.7, the $\circledast$ product on $\Sigma_{1}$ may be identified with the cup product because the diagram in \$13.6 commutes.

13.9. Based loop space. Let $\Omega=\Omega_{x_{0}} \subset \Lambda$ denote the space of loops in $M$ that are based at $x_{0}$. Suppose as above that all geodesics on $M$ are simply periodic with the same prime length $\ell$. Since the index growth is maximal, cf. (12.1.1), the index of each critical point in $\Omega$ is the same as that in $\Lambda$, cf. Lemma 6.4. The critical set $\Sigma_{r}^{\Omega} \subset \Omega$ at level $r \ell$ is parametrized by the unit sphere $S^{n-1} \subset T_{x_{0}} M$. The arguments of the preceding section may be applied to the based loop space with its product $\circledast(\mathrm{cf}$. Proposition 8.5) and we conclude that the cohomology algebra $\left(H^{*}\left(\Omega_{x_{0}}, x_{0}\right), \circledast\right)$ is filtered by the energy and the associated graded algebra is isomorphic to the polynomial algebra $H^{*}\left(\Sigma_{1}^{\Omega}\right)[T]$ where $\operatorname{deg}(T)=b=\lambda_{1}+n-1$ and where $H^{*}\left(\Sigma_{1}^{\Omega}\right)$ is the cohomology algebra of the sphere $S^{n-1}$. The restriction mapping $H^{*}(\Lambda) \rightarrow H^{*}(\Omega)$ induces the mapping on the associated graded algebras

$$
H^{*}(S M)[T] \rightarrow H^{*}\left(S^{n-1}\right)[T]
$$


which is determined by the restriction homomorphism

$$
H^{*}\left(\Sigma_{1}\right) \rightarrow H^{*}\left(\Sigma_{1}^{\Omega}\right) .
$$

If $M=S^{n}$ is the n-sphere, the restriction mapping (13.9.2) is surjective when $n$ is odd.

\section{Related products}

14.1. Composing the Künneth isomorphism with the action $S^{1} \times \Lambda \rightarrow \Lambda$ gives a map

$$
\Delta_{*}: H_{i}(\Lambda ; G) \rightarrow H_{i+1}(\Lambda ; G) \text { and } \Delta^{*}: H^{i}(\Lambda ; G) \rightarrow H^{i-1}(\Lambda ; G)
$$

for any coefficient group $G$. Then [CS] define $H_{i}(\Lambda) \times H_{j}(\Lambda) \stackrel{\{\cdot \cdot \cdot\}}{\longrightarrow} H_{i+j-n+1}(\Lambda)$ such that

$$
\{\sigma, \delta\}=(-1)^{|\sigma|} \Delta_{*}(\sigma * \delta)-(-1)^{|\sigma|} \Delta_{*}(\sigma) * \delta-\sigma * \Delta_{*}(\delta)
$$

where $|\sigma|=i-n$ if $\sigma \in H_{i}(\Lambda)$. They prove that the bracket is (graded) anti-commutative, it satisfies the (graded) Jacobi identity, and it is a derivation in each variable, that is,

(1) $\{\sigma, \tau\}=-(-1)^{(|\sigma|+1)(|\tau|+1)}\{\tau, \sigma\}$

(2) $\{\sigma,\{\tau, \omega\}\}=\{\{\sigma, \tau\}, \omega\}+(-1)^{(|\sigma|+1)(|\tau|+1)}\{\tau,\{\sigma, \omega\}\}$

(3) $\{\sigma, \tau * \omega\}=\{\sigma, \tau\} * \omega+(-1)^{|\tau|(|\sigma|+1)} \tau *\{\sigma, \omega\}$.

Since $\Delta_{*}$ preserves the energy, it follows that the bracket operation is also defined on the relative homology groups $\check{H}_{*}\left(\Lambda \leq a, \Lambda \leq a^{\prime} ; G\right)$ and it satisfies the energy estimates of Proposition 5.4 .

Similarly, we may define $H^{i}\left(\Lambda, \Lambda_{0}\right) \times H^{j}\left(\Lambda, \Lambda_{0}\right) \stackrel{\{\cdot \cdot \cdot\}}{\longrightarrow} H^{i+j+n-2}\left(\Lambda, \Lambda_{0}\right)$ by

$$
\{\tau, \omega\}=(-1)^{|\tau|} \Delta^{*}(\tau \circledast \omega)-(-1)^{|\tau|} \Delta^{*}(\tau) \circledast \omega-\tau \circledast \Delta^{*}(\omega)
$$

where $|\tau|=i+n-1$ if $\tau \in H^{i}(\Lambda)$.

14.2. Theorem. The cohomology bracket satisfies the following for any $\sigma, \tau, \omega \in H^{*}\left(\Lambda, \Lambda_{0}\right)$.

(A) $\{\tau, \omega\}=-(-1)^{(|\tau|+1)(|\omega|+1)}\{\omega, \tau\}$

(B) $\{\sigma,\{\tau, \omega\}\}=\{\{\sigma, \tau\}, \omega\}+(-1)^{(|\tau|+1)(|\omega|+1)}\{\tau,\{\sigma, \omega\}\}$

(C) $\{\sigma, \tau \circledast \omega\}=\{\sigma, \tau\} \circledast \omega+(-1)^{|\tau|(|\sigma|+1)} \tau \circledast\{\sigma, \omega\}$.

Proof. Part (A) follows directly from the definition. The proof of parts (B) and (C) will appear in Appendix D.

14.3. Nondegenerate case. As in Theorem 11.3, assume the manifold $M$ is orientable, $\gamma$ is a closed geodesic such that all of its iterates are nondegenerate, and assume the negative bundle $\Gamma_{r}$ is orientable for all $r$, cf. footnote 3. Let $a=L(\gamma)$. Assume $r \geq 2$. Let $\sigma_{r}, \bar{\sigma}_{r}, \tau_{r}, \bar{\tau}_{r}$ be the local (level) homology and cohomology classes described in equation (11.2.1). In the local level (co)homology group $H\left(\Lambda^{<r a} \cup \Sigma_{r}, \Lambda^{<r a}\right)$ we have:

$$
\begin{aligned}
\Delta_{*}\left(\sigma_{r}\right) & =r \bar{\sigma}_{r} ; & \Delta_{*}\left(\bar{\sigma}_{r}\right) & =0 \\
\Delta^{*}\left(\tau_{r}\right) & =r \bar{\tau}_{r} ; & \Delta^{*}\left(\bar{\tau}_{r}\right) & =0 .
\end{aligned}
$$


Using Theorem 11.3, if $\lambda_{j+k}=\lambda_{j+k}^{\min }$ then in $H_{*}\left(\Lambda^{<(j+k) a} \cup \Sigma_{j+k}, \Lambda^{<(j+k) a}\right)$ we have

$$
\begin{aligned}
& \left\{\sigma_{j}, \sigma_{k}\right\}=-\left(k+(-1)^{\left|\sigma_{1}\right|} j\right) \sigma_{j+k} \\
& \left\{\sigma_{j}, \bar{\sigma}_{k}\right\}=(-1)^{\left|\sigma_{1}\right|} k \bar{\sigma}_{j+k}
\end{aligned}
$$

while if $\lambda_{j+k}=\lambda_{j+k}^{\max }$ then in $H^{*}\left(\Lambda^{<(j+k) a} \cup \Sigma_{j+k}, \Lambda^{<(j+k) a}\right)$ we have

$$
\begin{aligned}
\left\{\tau_{j}, \tau_{k}\right\} & =\left(-k+(-1)^{\left|\tau_{1}\right|} j\right) \tau_{j+k} \\
\left\{\tau_{j}, \bar{\tau}_{k}\right\} & =(-1)^{\left|\tau_{1}\right|} k \bar{\tau}_{j+k} .
\end{aligned}
$$

14.4. Equivariant homology and cohomology. As in CS, one may consider the $T=$ $S^{1}$-equivariant homology $H_{*}^{T}(\Lambda)$ of the free loop space $\Lambda$. Let $E T \rightarrow B T$ be the classifying space and universal bundle for $T=S^{1}$; it is the limit of finite dimensional approximations $S^{2 n+1} \rightarrow \mathbb{C} P^{n}$ and let $\pi: \Lambda \times E T \rightarrow \Lambda_{T}=\Lambda \times_{T} E T$ be the Borel construction. There are Gysin (exact) sequences ([Sp] $\S 5.7$ p. 260) with coefficients in $\mathbb{Z}$,

$$
\begin{aligned}
& \longrightarrow H_{i+1}(\Lambda) \stackrel{\pi_{*}}{\longrightarrow} H_{i+1}^{T}(\Lambda) \longrightarrow H_{i-1}^{T}(\Lambda) \stackrel{\pi^{*}}{\longrightarrow} H_{i}(\Lambda) \longrightarrow \\
& \longrightarrow H_{T}^{i}(\Lambda) \underset{\pi^{*}}{\longrightarrow} H^{i}(\Lambda) \underset{\pi_{*}}{\longrightarrow} H^{i-1}(\Lambda) \longrightarrow H^{i+1}(\Lambda) \longrightarrow
\end{aligned}
$$

The Chas-Sullivan "string bracket" (homology) product on equivariant homology is defined to be $(-1)^{i-n}$ times the composition

$$
H_{i}^{T}(\Lambda) \times H_{j}^{T}(\Lambda) \stackrel{\pi^{*} \times \pi^{*}}{\longrightarrow} H_{i+1}(\Lambda) \times H_{j+1}(\Lambda) \stackrel{*}{\longrightarrow} H_{i+j+2-n}(\Lambda) \stackrel{\pi_{*}}{\longrightarrow} H_{i+j+2-n}^{T}(\Lambda),
$$

that is, $[\sigma, \delta]=(-1)^{|\sigma|} \pi_{*}\left(\pi^{*}(\sigma) * \pi^{*}(\delta)\right)$. The action of $T=S^{1}$ preserves the energy function, so the (homology) string bracket extends to products on relative homology $\check{H}_{*}^{T}\left(\Lambda \leq a, \Lambda \leq a^{\prime}\right)$ and on level homology $\check{H}_{*}^{T}\left(\Lambda^{\leq a}, \Lambda^{<a}\right)$ which satisfy the same energy estimates as those in Proposition 5.4.

Similarly the (cohomology) product $\circledast$ gives rise to a product in equivariant cohomology as $(-1)^{i+n-1}$ times the composition

$$
H_{T}^{i}(\Lambda) \times H_{T}^{j}(\Lambda) \stackrel{\pi^{*} \times \pi^{*}}{\longrightarrow} H^{i}(\Lambda) \times H^{j}(\Lambda) \stackrel{\circledast}{\longrightarrow} H^{i+j+n-1}(\Lambda) \stackrel{\pi_{*}}{\longrightarrow} H_{T}^{i+j+n-2}(\Lambda),
$$

or $\tau \odot \omega=(-1)^{|\tau|} \pi_{*}\left(\pi^{*}(\tau) \circledast \pi^{*}(\omega)\right)$. It also gives products in relative equivariant cohomology $\check{H}_{T}^{*}\left(\Lambda^{\leq a}, \Lambda^{\leq b}\right)$ with energy estimates as in Proposition 8.6 .

14.5. The string bracket is discussed in [CS], p. 24 in the case when $M$ is a surface of genus $>1$. When $n=2$ it gives a non-trivial map

$$
H_{0}^{T}(\Lambda) \times H_{0}^{T}(\Lambda) \underset{46}{\stackrel{[\cdot, \cdot])}{\longrightarrow}} H_{0}^{T}(\Lambda)
$$


which turns out to be a product discovered by Goldman [Go] and Wolpert [Wo]. In this case the equivariant cohomology product @ is also non-trivial in degree zero,

$$
H_{T}^{0}\left(\Lambda, \Lambda_{0}\right) \times H_{T}^{0}\left(\Lambda, \Lambda_{0}\right) \stackrel{\odot}{\longrightarrow} H_{T}^{0}\left(\Lambda, \Lambda_{0}\right)
$$

The group $H_{T}^{0}\left(\Lambda, \Lambda_{0}\right)$ can be identified with the set of maps from the set of free homotopy classes of loops in $M$ to the coefficient group $G$, which take the homotopy class of trivial loops to the identity element in $G$. We give a simple example: consider the case when $\alpha, \beta \in H_{T}^{0}\left(\Lambda, \Lambda_{0}\right)$ are given by $\cap A$ and $\cap B$ where $A$ and $B$ are disjoint simple closed loops in $M$. Assume that $M$ also supports loops $A^{\prime}$ and $B^{\prime}$ with

$$
\begin{aligned}
& A \cap A^{\prime}=B \cap B^{\prime}=1 \\
& A \cap B^{\prime}=B \cap A^{\prime}=A \cap B=0 .
\end{aligned}
$$

Let $C \in \Lambda$. By chasing through the definitions we see that the class

$$
\alpha \odot \beta \in H_{T}^{0}\left(\Lambda, \Lambda_{0}\right)
$$

has the following properties, when considered as a map from the set of free homotopy classes of loops in $M$ to $G$ :

(1) $\langle\alpha \odot \beta,[C]\rangle=0$ if $C$ is an embedded loop,

(2) $\langle\alpha \odot \beta,[C]\rangle=1$ if there exist loops $A^{\prime \prime}, B^{\prime \prime}$, homotopic to $A^{\prime}, B^{\prime}$ (respectively), which intersect transversally at the point $A^{\prime \prime}(0)=B^{\prime \prime}(0)$ such that $C$ is homotopic to the composed loop $A^{\prime \prime} \cdot B^{\prime \prime}$.

This product appears to be related to the Turaev cobracket described in [Chs], p. 27.

\section{Appendix A. Čech homology and cohomology}

A.1. Throughout this paper, the symbols $H_{i}$ and $H^{j}$ denote the singular homology and cohomology while $\check{H}_{i}$ and $\check{H}^{j}$ denote the Čech homology and cohomology as described, for example in [ES] §9, D] p. 339, [Br] p. 315 (Čech homology), and [Sp $§ 6.7$ Ex. 14 p. 327 (Čech cohomology).

The problem is that the space $\Lambda \leq a$ and even its finite dimensional approximation $\mathcal{M}_{N}^{\leq a}$ might be pathological if $a$ is a critical value of the function $F$. However, for each regular value $a+\epsilon$ the space $\Lambda^{\leq a+\epsilon}$ has the homotopy type of a finite simplicial complex. Thus one might hope to describe the homology and cohomology of $\Lambda \leq a$ using a limiting process. The Cech homology and cohomology are better behaved under limiting processes than the singular homology and cohomology. Unfortunately, the Cech homology does not always satisfy the exactness axiom for a homology theory (although the Čech cohomology does satisfy the exactness axiom). These issues are explained in detail in [ES]. We now review the relevant properties of these homology theories that are used in this article. 
A.2. Let $G$ be an Abelian group. If $A \subset X$ are topological spaces then the composition of any two homomorphisms in the homology sequence for the pair $\check{H}_{*}(X, A ; G)$ is always zero. If $X$ and $A$ are compact and if $G$ is finite or if $G$ is a field then the homology sequence for $\check{H}_{*}(X, A ; G)$ is exact.

If a topological space $X$ has the homotopy type of a finite simplicial complex then the natural transformations $H_{j}(X ; G) \rightarrow \check{H}_{j}(X ; G)$ and $\check{H}^{j}(X ; G) \rightarrow H^{j}(X ; G)$ are isomorphisms for all $j$.

By [Ha Thm 3.33, if a topological space $X$ is an increasing union of subspaces $X_{1} \subset$ $X_{2} \subset \cdots$ and if every compact subset $K \subset X$ is contained in some $X_{n}$ then for all $j$ the inclusions $X_{n} \rightarrow X$ induces isomorphisms

$$
H_{j}(X ; G) \cong \lim _{\longrightarrow} H_{j}\left(X_{n} ; G\right) \text { and } \check{H}_{j}(X ; G) \cong \lim _{\longrightarrow} \check{H}_{j}\left(X_{n} ; G\right) .
$$

A.3. Let $A$ be a closed subset of a paracompact Hausdorff space $X$. Let $U_{1} \supset U_{2} \supset \cdots$ be a sequence of subsets of $X$ such that $\cap_{n=1}^{\infty} U_{n}=A$. Then the following table describes sufficient conditions that

$$
\check{H}^{q}(A ; G) \cong \lim _{\longrightarrow} \check{H}^{q}\left(U_{n} ; G\right) \text { and } \check{H}_{q}(A ; G) \cong \lim _{\leftarrow} \check{H}_{q}\left(U_{n} ; G\right) .
$$

\begin{tabular}{|c||c|c|}
\hline & $U_{n}$ open & $U_{n}$ closed \\
\hline \hline cohomology & no restriction & $X$ is compact \\
homology & $\mathrm{X}$ is a manifold & $X$ is compact \\
\hline
\end{tabular}

These facts are classical and the proofs may be found in the textbooks, for example [ES] $\S I X, \S X,[\mathrm{Sp}] \S 6.6$ Thm 2, Thm 6; [D] VIII $\S 6.18, \S 13.4, \S 13.16$; and [Br]. (By [Sp] $\S 6.8$ Cor. 8, the Cech cohomology coincides with the Alexander-Spanier cohomology on the class of paracompact Hausdorff spaces.)

For the remainder of this Appendix, continue with the notation $M, \Lambda, F, \Sigma$ of $\$ 2$.

A.4. Lemma. Let $G$ be an Abelian group and let $a \in \mathbb{R}$. Then the natural homomorphisms

$$
H_{*}(\Lambda ; G) \rightarrow \check{H}_{*}(\Lambda ; G) \text { and } H_{*}\left(\Lambda^{<a} ; G\right) \rightarrow \check{H}_{*}\left(\Lambda^{<a} ; G\right)
$$

are isomorphisms. If $a \in \mathbb{R}$ is a regular value of $F$, or if a is a nondegenerate critical value of $F$ in the sense of Bott, then the morphism $H_{*}\left(\Lambda^{\leq a} ; G\right) \rightarrow \check{H}_{*}\left(\Lambda^{\leq a} ; G\right)$ is an isomorphism. The same statements hold for Čech cohomology.

Proof. This follows from Proposition 3.3: the space $\Lambda^{<a}$ has the homotopy type of a finite dimensional manifold, and if $a$ is a regular value then $\Lambda \leq a$ is homotopy equivalent to a finite dimensional compact manifold with boundary.

A.5. Lemma. If $a^{\prime}<a \in \mathbb{R}$ then the inclusion $\Lambda^{\leq a} \rightarrow \Lambda^{\leq a+\epsilon}$ induces canonical isomorphisms

$$
\begin{aligned}
\check{H}_{i}\left(\Lambda^{\leq a} ; G\right) & \cong \lim _{0 \longleftarrow \epsilon} H_{i}\left(\Lambda^{<a+\epsilon} ; G\right) \\
\check{H}_{i}\left(\Lambda^{\leq a}, \Lambda^{\leq a^{\prime}} ; G\right) & \cong \lim _{0 \leftarrow \epsilon} H_{i}\left(\Lambda^{<a+\epsilon}, \Lambda^{<a^{\prime}+\epsilon} ; G\right)
\end{aligned}
$$


with Čech homology on the left and singular homology on the right. If $G$ is a field, $\alpha \in$ $H_{i}(\Lambda ; G)$ and if $a=\operatorname{cr}(\alpha)$ is its critical value (\$4) then there exists $\omega \in \check{H}_{i}(\Lambda \leq a ; G)$ which maps to $\alpha$.

Proof. By Proposition 3.3 the space $\Lambda^{\leq a}$ is homotopy equivalent to the finite dimensional space $\mathcal{M}_{N}^{\leq a}$ which is contained in a manifold. Therefore

$$
\check{H}_{i}\left(\Lambda^{\leq a}\right) \cong H_{i}\left(\mathcal{M}_{N}^{\leq a}\right)=\lim _{0 \longleftarrow \epsilon} H_{i}\left(\mathcal{M}_{N}^{<a+\epsilon}\right) \cong \lim _{0 \longleftarrow \epsilon} H_{i}\left(\Lambda^{\leq a+\epsilon}\right)
$$

which proves A.5.1). The relative case A.5.2) is similar. Now suppose $G$ is a field and let $b_{n} \downarrow a=\operatorname{cr}(\alpha)$ be a convergent sequence of regular values of $F$. Then $\check{H}_{i}(\Lambda \leq a ; G)$ is the limit of the sequence of finite dimensional vector spaces

$$
H_{i}\left(\Lambda^{\leq b_{1}}\right) \leftarrow H_{i}\left(\Lambda^{\leq b_{2}}\right) \leftarrow H_{i}\left(\Lambda^{\leq b_{3}}\right) \leftarrow \cdots
$$

and for each $n \geq 1$ there is an element $\omega_{n} \in H_{i}\left(\Lambda^{\leq b_{n}}\right)$ that maps to $\alpha$. Let $H_{n}=$ Image $\left(H_{i}\left(\Lambda^{\leq b_{n}} ; G\right) \rightarrow H_{i}\left(\Lambda^{\leq b_{1}} ; G\right)\right)$. These form a decreasing chain of finite dimensional vector spaces which therefore stabilize after some finite point, say,

$$
H_{N}=\operatorname{Image}\left(H_{i}\left(\Lambda^{\leq b_{N}} ; G\right) \rightarrow H_{i}\left(\Lambda^{\leq b_{1}} ; G\right)\right)=\cap_{n=1}^{\infty} H_{n}=\check{H}_{i}\left(\Lambda^{\leq a} ; G\right) .
$$

It then suffices to take $\omega \in H_{N}$ to be the image of $\omega_{N} \in H_{i}\left(\Lambda^{\leq b_{N}} ; G\right)$.

A.6. Proposition. Fix $c \in \mathbb{R}$. Let $U \subset \Lambda$ be a neighborhood of $\Sigma^{=c}$. The inclusions

$$
\left(\Lambda^{<c} \cup \Sigma^{=c}\right) \cap U \hookrightarrow \Lambda^{<c} \cup \Sigma^{=c} \hookrightarrow \Lambda^{\leq c}
$$

induce isomorphisms on $\check{C}$ ech homology,

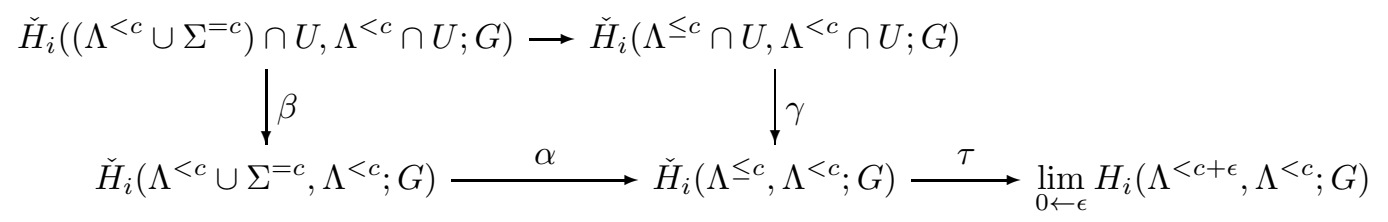

Proof. It follows from excision that the relative homology group $\check{H}_{i}\left(\left(\Lambda^{<c} \cup \Sigma^{c}\right) \cap U, \Lambda^{<c} \cap U\right)$ is independent of $U$. Taking $U=\Lambda$ gives the isomorphism $\beta$. The same argument applies to the isomorphism $\gamma$. The mapping $\tau$ is an isomorphism by \$.3. Finally, the mapping $\alpha$ is an isomorphism because the inclusion $\left(\Lambda^{<c} \cup \Sigma^{c}, \Lambda^{<c}\right) \rightarrow\left(\Lambda^{\leq c}, \Lambda^{<c}\right)$ is a homotopy equivalence. A homotopy inverse is given by the time $t$ flow $\psi_{t}: \Lambda \leq c \rightarrow \Lambda \leq c$ of the vector field $-\operatorname{grad}(F)$, for any choice of $t>0$ (cf. [K] $\S 1,[\mathrm{C}] \S \mathrm{I} .3$ ).

\section{Appendix B. Thom isomorphisms}

B.1. The constructions in this paper necessitate the use of various relative versions of the Thom isomorphism for finite and infinite dimensional spaces in singular and Čech homology and cohomology. In this section we review these standard facts. 
Recall that a neighborhood $N$ of a closed subset $X$ of a topological space $Y$ is a tubular neighborhood if there exists a finite dimensional ("normal") real vector bundle $\pi: E \rightarrow X$, and a homeomorphism $\psi: E \rightarrow N \subset Y$ which takes the zero section to $X$ by the identity mapping. In this case, excising $Y-N$ gives an isomorphism

$$
H(E, E-X ; G) \cong H(N, N-X ; G) \cong H(Y, Y-X ; G)
$$

where $H$ denotes either singular homology or cohomology (with coefficients in an Abelian group $G$ ) and where $E-X$ is the complement of the zero section. Let us take the coefficient group to be $G=\mathbb{Z}$ if the normal bundle $E$ is orientable (in which case we fix an orientation), and $G=\mathbb{Z} /(2)$ otherwise. The Thom class

$$
\mu_{E} \in H^{n}(E, E-X ; G)
$$

is the unique cohomology class which restricts to the chosen homology generator of each fiber $\pi^{-1}(x)$. The cup product with this class gives the Thom isomorphism in cohomology,

$$
H^{i}(X ; G) \cong H^{i}(E ; G) \rightarrow H^{i+n}(E, E-X ; G) \cong H^{i+n}(Y, Y-X ; G)
$$

and the cap product with this class gives the Thom isomorphism in homology,

$$
H_{i}(X ; G) \cong H_{i}(E ; G) \leftarrow H_{i+n}(E, E-X ; G) \cong H_{i+n}(Y, Y-X ; G) .
$$

See [Spanier] Chapt. 5 Sec. 7 p. 259. The same results hold for Čech homology and cohomology. We need to establish relative versions of these isomorphisms.

As usual we take the coefficient group $G$ to be either $\mathbb{Z}$ or $\mathbb{Z} /(2)$.

B.2. Proposition. Let $A \subset X$ be closed subsets of a topological space $Y$. Assume that $X$ has a tubular neighborhood $N$ in $Y$ corresponding to a homeomorphism $\phi: E \rightarrow N$ of a normal bundle $E \rightarrow X$ of fiber dimension $n$. If $E$ is orientable then choose an orientation and set $G=\mathbb{Z}$, otherwise set $G=\mathbb{Z} /(2)$. Then the Thom isomorphism induces an isomorphism

$$
H^{i}(X, X-A ; G) \cong H^{i+n}(Y, Y-A ; G)
$$

in singular cohomology, and an isomorphism

$$
H_{i}(X, X-A ; G) \cong H_{i+n}(Y, Y-A ; G)
$$

in singular homology. Taking $A=X$ gives Gysin homomorphisms

$$
\begin{aligned}
H^{i}(X ; G) & \cong H^{i+n}(Y, Y-X ; G) \rightarrow H^{i+n}(Y ; G) \\
H_{i+n}(Y ; G) & \rightarrow H_{i+n}(Y, Y-X ; G) \cong H_{i}(X ; G)
\end{aligned}
$$

denoted $h_{!}$and $h$ ! respectively, where $h: X \rightarrow Y$ denotes the inclusion. If $U \subset Y$ is open then taking $A=X-X \cap U$ gives Gysin homomorphisms

$$
\begin{aligned}
H^{i}(X, X \cap U ; G) & \cong H^{i+n}(Y, Y-A ; G) \rightarrow H^{i+n}(Y, U ; G) \\
H_{i+n}(Y, U ; G) & \rightarrow H_{i+n}(Y, Y-A ; G) \cong H_{i}(X, X \cap U ; G)
\end{aligned}
$$


Proof. We will describe the argument for (B.2.1); the argument for (B.2.2) is the same, with the arrows reversed. We suppress the coefficient group $G$ in order to simplify the notation in the following argument. Let $E^{A}=\pi^{-1}(X-A) \subset E$ and let $E^{0}=E-X$. The sets $E^{0}, E^{A}$ are open in $X$ so they form an excisive pair 6 giving the excision isomorphism

$$
H^{i}\left(E^{0} \cup E^{A}, E^{0}\right) \stackrel{\cong}{\longrightarrow}\left(E^{A}, E^{0} \cap E^{A}\right) .
$$

The cup product with the Thom class $\mu_{E} \in H^{n}\left(E, E^{0} ; \mathbb{Z}\right)$ gives a mapping

$$
H^{i}(X, X-A) \cong H^{i}\left(E, E^{A}\right) \rightarrow H^{i+n}\left(E, E^{0} \cup E^{A}\right)
$$

which we claim is an isomorphism. This follows from the five lemma and the exact sequence of the triple

$$
E^{0} \subset\left(E^{0} \cup E^{A}\right) \subset E .
$$

In fact, the following diagram commutes:

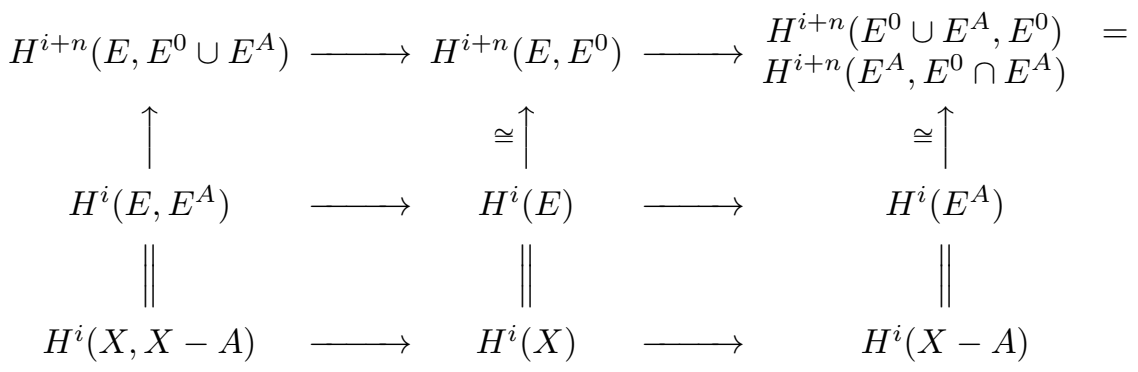

FiguRE 9 .

so the left hand vertical mapping is an isomorphism. Since $A$ is closed in $Y$ we may excise $Y-N$ from $Y-A$ to obtain an isomorphism

$$
H^{i+n}(Y, Y-A) \cong H^{i+n}(N, N-A) \cong H^{i+n}\left(E, E^{0} \cup E^{A}\right) .
$$

B.3. We will also need in $\$ 7$ the following standard facts concerning the Thom isomorphism. Suppose $E_{1} \rightarrow A$ and $E_{2} \rightarrow A$ are oriented vector bundles of ranks $d_{1}$ and $d_{2}$. If both are oriented let $G=\mathbb{Z}$ (and choose orientations of each), otherwise let $G=\mathbb{Z} /(2)$ be the coefficient group for homology. Let $E=E_{1} \oplus E_{2}$. The diagram of projections

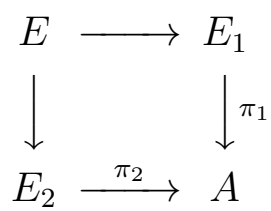

gives identifications $E \cong \pi_{1}^{*}\left(E_{2}\right) \cong \pi_{2}^{*}\left(E_{1}\right)$ of the total space $E$ as a vector bundle $\pi_{1}^{*}\left(E_{2}\right)$ over $E_{1}$ (resp. as a vector bundle $\pi_{2}^{*}\left(E_{1}\right)$ over $\left.E_{2}\right)$. The Thom class $\mu_{2} \in H^{d_{2}}\left(E_{2}, E_{2}-A\right)$

\footnotetext{
${ }^{6}$ A pair $A, B \subset X$ is excisive if $A \cup B=A^{o} \cup B^{o}$ where $A^{o}$ denotes the relative interior of $A$ in $A \cup B$, cf. Sp] p. 188
} 
pulls up to a class

$$
\pi_{1}^{*}\left(\mu_{2}\right) \in H^{d_{2}}\left(E, E-E_{1}\right)
$$

and similarly with the indices reversed. Then the relative cup product $H^{d_{1}}\left(E, E-E_{1}\right) \times H^{d_{2}}\left(E, E-E_{2}\right) \rightarrow H^{d_{1}+d_{2}}\left(E,\left(E-E_{1}\right) \cup\left(E-E_{2}\right)\right)=H^{d_{1}+d_{2}}(E, E-A)$ takes $\left(\pi_{1}^{*}\left(\mu_{2}\right), \pi_{2}^{*}\left(\mu_{1}\right)\right)$ to the Thom class

$$
\mu_{E}=\pi_{1}^{*}\left(\mu_{2}\right) \smile \pi_{2}^{*}\left(\mu_{1}\right) .
$$

Consequently the Thom isomorphism for $E$ is the composition of the Thom isomorphisms

$$
H^{i}(A) \underset{\smile \mu_{1}}{\longrightarrow} H^{i+d_{1}}\left(E_{1}, E_{1}-A\right) \underset{\mu_{2}}{\longrightarrow} H^{i+d_{1}+d_{2}}(E, E-A) .
$$

B.4. Corollary. In the situation of Proposition B.2, suppose that $A \subset X \subset Y$ are closed sets, that $A$ has a tubular neighborhood in $X$ with oriented normal bundle $\Upsilon$ of rank $m$, and suppose $X$ has a tubular neighborhood in $Y$ with oriented normal bundle $E$ of rank $n$. Then $\mu_{E \oplus \Upsilon}=\pi_{\Upsilon}^{*}\left(\mu_{E}\right) \smile \pi_{E}^{*}\left(\mu_{\Upsilon}\right)$ is a Thom class in $H^{n+m}(E \oplus \Upsilon, E \oplus \Upsilon-A)$ and the composition of Thom isomorphisms across the bottom, in the following diagram

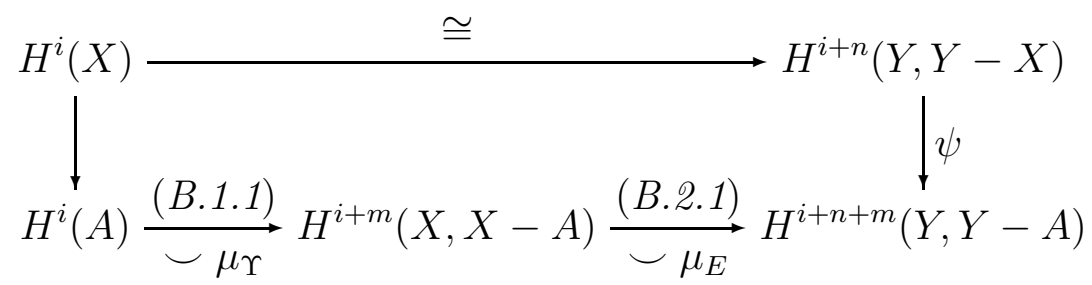

is the Thom isomorphism $\smile\left(\mu_{E \oplus \Upsilon}\right)$. The diagram gives rise to a Gysin homomorphism $\psi: H^{r}(Y, Y-X) \rightarrow H^{r+m}(Y, Y-A)$ which may be interpreted as the cup product with the Thom class

$$
\mu_{\Upsilon} \in H^{m}(\Upsilon, \Upsilon-A) \cong H^{m}(X, X-A) \cong H^{m}\left(E, E-\pi_{E}^{-1}(A)\right)
$$

in the following sequence of homomorphisms

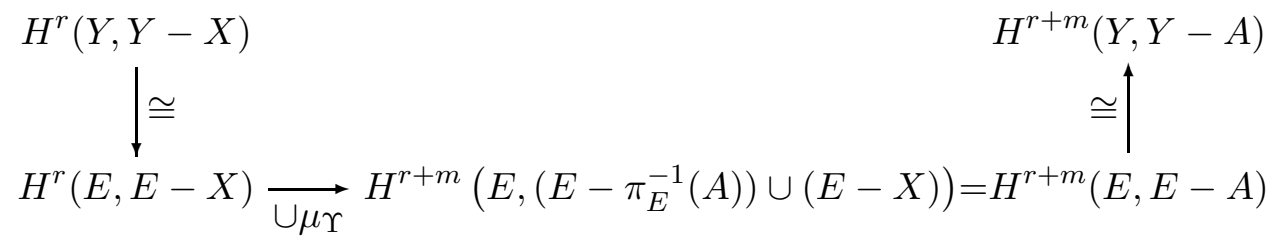

\section{Appendix C. Theorems of Morse and Bott}

C.1. We have the following theorems of Morse and Bott [Mo1, [Bo2, [Mi], Bo1, [L], [K] Cor. 2.4.11, and $\$ 3.2,[\mathrm{R}]$. (By Lemma A.4 the homology groups that appear in the following theorem may be taken to be either Čech or singular.) 
C.2. Theorem. Let $X$ be a Riemannian Hilbert manifold and let $f: X \rightarrow \mathbb{R}$ be a smooth function that satisfies condition $C$. Let $\Sigma$ be a finite dimensional connected nondegenerate critical submanifold in the strong sense that the eigenvalues of $d^{2} f$ on the normal bundle of $\Sigma$ are bounded away from 0 . Let $\lambda<\infty$ be the index of $\Sigma$ and let $d<\infty$ be the dimension of $\Sigma$. Let $c=f(\Sigma)$ be the critical value. Suppose there is a smooth connected manifold $V$ with

$$
\operatorname{dim}(V)=\operatorname{dim}(\Sigma)+\lambda
$$

and smooth embeddings

$$
\Sigma \underset{\sigma}{\longrightarrow} V \underset{\rho}{\longrightarrow} X
$$

so that $\rho \circ \sigma: \Sigma \rightarrow \Sigma$ is the identity and $f \circ \rho(x)<c$ whenever $x \in V-\Sigma$. Then $\rho$ induces an isomorphism

$$
H_{i}(V, V-\Sigma ; G) \stackrel{\cong}{\longrightarrow} H_{i}\left(X^{<c} \cup \Sigma, X^{<c} ; G\right)
$$

for any coefficient group $G$ and for all $i \geq 0$. In fact, $\rho$ induces a local diffeomorphism of pairs $(V, V-\Sigma) \cong\left(\Sigma^{-}, \Sigma^{-}-\Sigma\right)$, where $\Sigma^{-}$is defined below.

Composing with the Thom isomorphism (B.1.2) gives a further isomorphism

$$
H_{i}(V, V-\Sigma ; G) \cong H_{i-\lambda}(\Sigma ; G)
$$

where $G=\mathbb{Z}$ if the normal bundle of $\Sigma$ in $V$ is orientable, and $G=\mathbb{Z} /(2)$ otherwise.

C.3. Proof. This essentially follows from Theorem 7.3 (p. 72) of [C] or Corollary 2.4.8 and Proposition 2.4 .9 of $[\mathrm{K}]$. The tangent bundle $T X \mid \Sigma$ decomposes into an orthogonal sum of vector bundles $\Gamma^{+} \oplus \Gamma^{0} \oplus \Gamma^{-}$spanned by the positive, null, and negative eigenvectors (respectively) of the self adjoint operator associated to $d^{2} f$. The inclusion $\Sigma \rightarrow X$ induces an isomorphism $T \Sigma \cong \Gamma^{0}$ so we may identify the normal bundle of $\Sigma$ in $X$ with $\Gamma^{+} \oplus \Gamma^{-}$.

For $\epsilon$ sufficiently small the restriction of the exponential map

$$
\exp :\left(\Gamma^{+} \oplus \Gamma^{-}\right)_{\epsilon} \rightarrow X
$$

is a homeomorphism onto some neighborhood $U \subset X$. Let $\Sigma^{-}=\exp \left(\Gamma_{\epsilon}^{-}\right) \subset X$. This submanifold is often described as "the unstable manifold that hangs down from $\Sigma$," for if $\epsilon$ is sufficiently small and if $0 \neq a \in \Gamma_{\epsilon}^{-}$then $f(\exp (a))<c$. Its tangent bundle, when restricted to $\Sigma$, is

$$
T \Sigma^{-} \mid \Sigma=\Gamma^{0} \oplus \Gamma^{-}
$$

The projection $\Gamma^{+} \oplus \Gamma^{-} \rightarrow \Gamma^{-}$induces a projection $\pi: U \rightarrow \Sigma^{-}$which is homotopic to the identity by the homotopy

$$
\pi_{t}(\exp (a \oplus b))=\exp (t a \oplus b)
$$

where $t \in[0,1], a \in \Gamma_{\epsilon}^{+}, b \in \Gamma_{\epsilon}^{-}$, and where $\pi_{1}$ is the identity and $\pi_{0}=\pi$. Moreover the kernel of the differential $d \pi(x): T_{x} X \rightarrow T_{x} \Sigma^{-}$at any point $x \in \Sigma$ is precisely the positive 
eigenspace, $\Gamma_{x}^{+} \subset T_{x} X$. Let us identify the manifold $V$ with its image $\rho(V) \subset X$ so that $T V \mid \Sigma \subset \Gamma^{0} \oplus \Gamma^{-}$. It follows that the restriction of $\pi$

$$
\pi: V \cap U \rightarrow \Sigma^{-}
$$

has nonvanishing differential at every point $x \in \Sigma \subset V$ and consequently the mapping (C.3.1) is a diffeomorphism in some neighborhood of $\Sigma$. It follows that $\pi$ induces an isomorphism

$$
\pi_{*}: H_{i}(V, V-\Sigma) \rightarrow H_{i}\left(\Sigma^{-}, \Sigma^{-}-\Sigma\right) \cong H_{i}\left(D \Gamma^{-}, \partial D \Gamma^{-}\right)
$$

where $D \Gamma^{-}$denotes a sufficiently small disk bundle in $\Gamma^{-}$and $\partial D \Gamma^{-}$is its boundary. On the other hand, by Morse theory (the above mentioned Theorem 7.3 of [C] or Proposition 2.4.9 of $[\mathrm{K}]$ ), the space $U^{\leq c+\delta}$ has the homotopy type of the adjunction space $U^{\leq c-\delta} \cup_{\partial D \Gamma^{-}} D \Gamma^{-}$. This gives the standard isomorphism of Morse theory,

$$
H_{i}\left(D \Gamma^{-}, \partial D \Gamma^{-}\right) \cong H_{i}\left(U^{\leq c+\delta}, U^{\leq c-\delta}\right) .
$$

All these isomorphisms fit together in a commutative diagram:

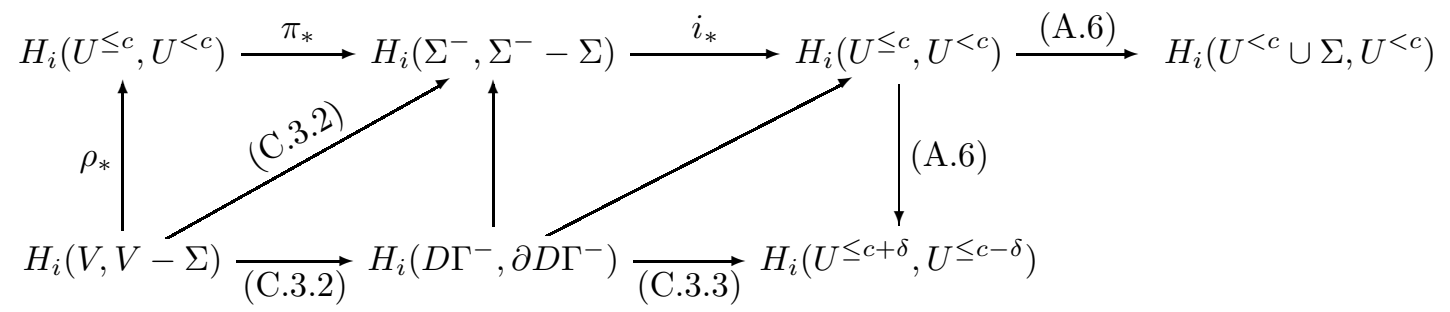

Each of the arrows labeled by an equation number is an isomorphism, so $i_{*}$ is an isomorphism. But $i_{*} \pi_{*}$ is the identity, so $\pi_{*}$ is also an isomorphism, hence also $\rho_{*}$.

\section{Appendix D. Proof of Theorem 14.2}

D.1. The proof of Theorem 14.2 involves a second construction of the cohomology bracket, along the same lines as the definition of the $\circledast$ product. As in $\S 2$ let $\Lambda$ be the free loop space of mappings $x: \mathbb{R} / \mathbb{Z} \rightarrow M$ (or $x:[0,1] \rightarrow M$ ). For the purposes of this appendix only, let $\widehat{\Lambda}$ be the free loop space of $H^{1}$ mappings $\mathbb{R} / 2 \mathbb{Z} \rightarrow M$ (or $[0,2] \rightarrow M$ ). If $x, y \in \Lambda$, if $s \in \mathbb{R} / \mathbb{Z}$ (or $s \in[0,1]$ ) and if $x(0)=y(s)$, define $x \cdot{ }_{s} y \in \widehat{\Lambda}$ (see Figure 10) by

$$
x \cdot{ }_{s} y(t)= \begin{cases}y(t) & \text { if } 0 \leq t \leq s \\ x(t-s) & \text { if } s \leq t \leq 1+s . \\ y(t)=y(t-1) & \text { if } 1+s \leq t \leq 2\end{cases}
$$

Define $\{\Lambda, \Lambda\}$ to be the set of triples $(x, y, s) \in \Lambda \times \Lambda \times \mathbb{R} / 2 \mathbb{Z}$ such that

$$
\left\{\begin{array}{l}
x(0)=y(s) \quad \text { if } \quad 0 \leq s \leq 1 \\
y(0)=x(s) \text { if } \quad 1 \leq s \leq 2
\end{array}\right.
$$




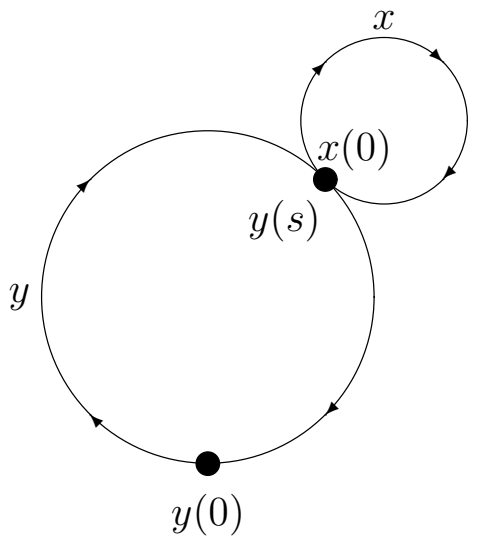

Figure 10. Joining two loops at time $s$

Define $\Phi_{1}:\{\Lambda, \Lambda\} \rightarrow \widehat{\Lambda}$ by $\Phi_{1}(x, y, s)= \begin{cases}x \cdot{ }_{s} y & \text { if } 0 \leq s \leq 1 \\ y \cdot{ }_{(s-1)} x & \text { if } 1 \leq s \leq 2 .\end{cases}$

We have embeddings

$$
\Lambda \times \Lambda \times \mathbb{R} /(2 \mathbb{Z}) \stackrel{h}{\longleftarrow}\{\Lambda, \Lambda\} \stackrel{\Phi}{\longrightarrow} \widehat{\Lambda} \times \mathbb{R} / 2 \mathbb{Z}
$$

where $\Phi(x, y, s)=\left(\Phi_{1}(x, y, s), s\right)$. The images $\Phi(\{\Lambda, \Lambda\})$ and $h(\{\Lambda, \Lambda\})$ have normal bundles and tubular neighborhoods and in fact they are given by the pull-back of the diagonal $\Delta$ under the mappings

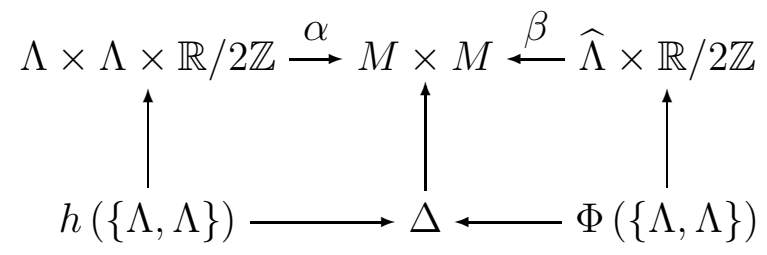

where

$$
\alpha(x, y, s)= \begin{cases}(x(0), y(s)) & \text { if } 0 \leq s \leq 1 \\ (x(s), y(0)) & \text { if } 1 \leq s \leq 2\end{cases}
$$

and $\beta(w, s)=(w(s), w(s+1))$. (Each half of $\{\Lambda, \Lambda\}$ has a smooth tubular neighborhood and normal bundle in $\Lambda \times \Lambda \times \mathbb{R} / 2 \mathbb{Z}$ but there is a "kink" where the two halves are joined so we only obtain a topological tubular neighborhood and normal bundle of $h(\{\Lambda, \Lambda\})$.) In particular,

$$
\Phi(\{\Lambda, \Lambda\})=\{(w, s) \in \widehat{\Lambda} \times \mathbb{R} / 2 \mathbb{Z}: w(s)=w(s \pm 1)\}
$$


D.2. We claim that the bracket $\{x, y\} \in H_{\operatorname{deg}(x)+\operatorname{deg}(y)-n+1}(\Lambda)$ is obtained by passing from left to right in (D.1.1) , i. e., it is the image of $x \times y \times[\mathbb{R} / 2 \mathbb{Z}]$ under the composition

$$
\begin{gathered}
H_{i}(\Lambda) \times H_{j}(\Lambda) \times H_{1}(\mathbb{R} / 2 \mathbb{Z}) \stackrel{\epsilon \times}{\longrightarrow} H_{i+j+1}(\Lambda \times \Lambda \times \mathbb{R} / 2 \mathbb{Z}) \\
h^{!} \downarrow(\underline{\mathrm{B} .2 .4}) \\
H_{i+j-n+1}(\widehat{\Lambda}) \stackrel{\Phi_{1}}{\longleftarrow} H_{i+j+1-n}(\{\Lambda, \Lambda\})
\end{gathered}
$$

where $\operatorname{deg}(x)=i, \operatorname{deg}(y)=j, \epsilon=(-1)^{n(n-j-1)}$, and $[\mathbb{R} / 2 \mathbb{Z}] \in H_{1}(\mathbb{R} / 2 \mathbb{Z})$ denotes the orientation class. First we show this agrees with the definition of $\{x, y\}$ in $[\mathrm{CS}$.

The projection $\pi:\{\Lambda, \Lambda\} \rightarrow \mathbb{R} / 2 \mathbb{Z}$ is locally trivial and $\pi^{-1}(0) \cong \pi^{-1}(1) \cong \mathcal{F}$ is the figure eight space. Let $\{\Lambda, \Lambda\}_{[0,1]}=\pi^{-1}([0,1])$ and $\partial\{\Lambda, \Lambda\}_{[0,1]}=\pi^{-1}(\{0\} \cup\{1\})$. Then the bracket product in [CS] is a sum of two terms,

$$
\{x, y\}=x \vee y-(-1)^{(i-n+1)(j-n+1)} y \diamond x
$$

(but [CS] use a $*$ rather than a $\varnothing$ ) which may be identified as the two images of

$$
h^{!}(\epsilon x \times y \times[\mathbb{R} / 2 \mathbb{Z}]) \in H_{i+j-n+1}(\{\Lambda, \Lambda\})
$$

in

$$
H_{i+j-n+1}\left(\Phi\left(\{\Lambda, \Lambda\}_{[0,1]}\right), \partial \Phi\left(\{\Lambda, \Lambda\}_{[0,1]}\right)\right) \text { and } H_{i+j-n+1}\left(\Phi\left(\{\Lambda, \Lambda\}_{[1,2]}\right), \partial \Phi\left(\{\Lambda, \Lambda\}_{[1,2]}\right)\right)
$$

respectively. The projection to $\widehat{\Lambda}$ adds these together (with the appropriate sign).

The proof that the construction of $\S \mathrm{D} .2$ agrees with (14.1.1) is essentially the same as the proof of Corollary 5.3 in [CS]. Using this fact, the proof of $(1),(2),(3)$ in $\$ 14.1$ is then the same as in [CS] $\S 4$.

D.3. In this paragraph we define the reparametrization function

$$
\widehat{J}: \widehat{\Lambda} \times \mathbb{R} / 2 \mathbb{Z} \times[0,2] \rightarrow \widehat{\Lambda} \times \mathbb{R} / 2 \mathbb{Z}
$$

that is analogous to the function $J$ of $\oint 8.1$. First some notation. For $r \in[0,2]$ let $\widehat{\theta}_{1 \rightarrow r}$ : $[0,2] \rightarrow[0,2]$ be the piecewise linear function taking $0 \mapsto 0,1 \mapsto r$, and $2 \mapsto 2$. It is just the function $\theta$ of 98.1 , but the domain and range have been stretched to $[0,2]$. For any real number $s$ define the translation $\chi_{s}: \mathbb{R} / 2 \mathbb{Z} \rightarrow R Z$ by $\chi_{s}(t)=t+s$. Define

$$
\widehat{J}(w, s, r)=\left(w \circ \chi_{s} \circ \theta_{1 \rightarrow r} \circ \chi_{-s}, s\right)
$$

and set $\widehat{J}_{r}(w, s)=\widehat{J}(w, s, r)$. In analogy with our notation for $\mathcal{F}$ in $\S 8.1$ let $\{\Lambda, \Lambda\}^{>0,>0}$ be the set of $(x, y, s) \in\{\Lambda, \Lambda\}$ such that $F(x)>0$ and $F(y)>0$. Let $\widehat{\Lambda}_{0}=\widehat{\Lambda}^{=0}$ denote the constant loops in $\widehat{\Lambda}$. We claim that $\widehat{J}$ takes both of the following sets

$$
\widehat{\Lambda}_{0} \times \mathbb{R} / 2 \mathbb{Z} \times[0,2] \text { and } \widehat{\Lambda} \times \mathbb{R} / 2 \mathbb{Z} \times \partial[0,2]
$$

into the set $\widehat{\Lambda} \times \mathbb{R} / 2 \mathbb{Z}-\Phi\left(\{\Lambda, \Lambda\}^{>0,>0}\right)$. 
This is obvious for the first of these sets, while the verification for the second set involves four cases: $s \leq 1$ or $s \geq 1$ vs. $r=0$ or $r=2$. In each case the function $\chi_{s} \circ \widehat{\theta}_{1 \rightarrow r} \circ \chi_{-s}$ is constant, with value $s$, on the interval $[s, s+1](\bmod 2)$. Therefore $J(w, s, r)=(\gamma, s)$ where $\gamma=x \cdot s y(s \leq 1)$ or $\gamma=x \cdot(s-1) y(s \geq 1)$ and either $x$ or $y$ is a constant loop.

D.4. The geometric construction of the cohomology bracket is the following composition,

$$
\begin{gathered}
H^{i}\left(\Lambda, \Lambda_{0}\right) \times H^{j}\left(\Lambda, \Lambda_{0}\right) \times H^{0}(\mathbb{R} / 2 \mathbb{Z}) \stackrel{\epsilon \times}{\longrightarrow} H^{i+j}\left(\left(\Lambda, \Lambda_{0}\right) \times\left(\Lambda, \Lambda_{0}\right) \times \mathbb{R} / 2 \mathbb{Z}\right) \\
h^{*} \downarrow \\
H^{i+j}\left(\{\Lambda, \Lambda\},\{\Lambda, \Lambda\}-\{\Lambda, \Lambda\}^{>0,>0}\right) \\
\Phi_{!} \downarrow(\underline{\mathrm{B} .2 .1}) \\
H^{i+j+n}\left(\widehat{\Lambda} \times \mathbb{R} / 2 \mathbb{Z}, \widehat{\Lambda} \times \mathbb{R} / 2 \mathbb{Z}-\Phi\left(\{\Lambda, \Lambda\}^{>0,>0}\right)\right) \\
\widehat{J}^{*} \downarrow \\
\left.H^{i+j+n-2}\left(\widehat{\Lambda}, \widehat{\Lambda}_{0}\right)\right) \stackrel{\pi_{!}}{\longleftarrow} H^{i+j+n}\left(\left(\widehat{\Lambda}, \widehat{\Lambda}_{0}\right) \times \mathbb{R} / 2 \mathbb{Z} \times([0,2], \partial[0,2])\right)
\end{gathered}
$$

where $\pi$ denotes the projection to $\widehat{\Lambda}$. So the bracket is obtained by passing from left to right in the following diagram,

$$
\Lambda \times \Lambda \times \mathbb{R} / 2 \mathbb{Z} \stackrel{h}{\longleftarrow}\{\Lambda, \Lambda\} \stackrel{\Phi}{\longrightarrow} \widehat{\Lambda} \times \mathbb{R} / 2 \mathbb{Z} \stackrel{\widehat{J}}{\longleftarrow} \widehat{\Lambda} \times \mathbb{R} / 2 \mathbb{Z} \times[0,2] \longrightarrow \widehat{\Lambda}
$$

D.5. For $r \in[0,2]$ set $\widehat{J}_{r}(w, s)=\widehat{J}(w, s, r)$. Let $\mathcal{T}:\{\Lambda, \Lambda\} \rightarrow\{\Lambda, \Lambda\}$ by $T(x, y, s)=$ $(y, x, s+1)$ and (by abuse of notation) set $\mathcal{T}: \widehat{\Lambda} \times \mathbb{R} / 2 \mathbb{Z} \rightarrow \widehat{\Lambda} \times \mathbb{R} / 2 \mathbb{Z}$ by $\mathcal{T}(z, s)=$ $\left(z \circ \chi_{1}, s+1\right)$. (So $\mathcal{T}$ moves the basepoint half way around the loop.) Then the following diagram commutes:

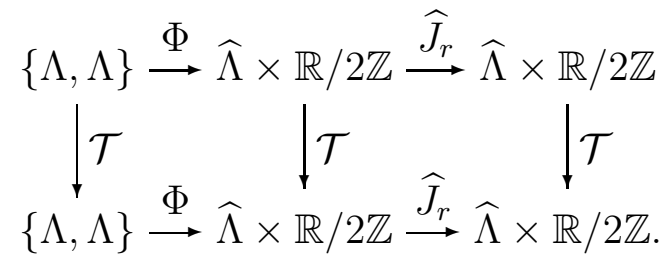

As in $\$$ D.2 the bracket is a sum of two terms,

$$
\{x, y\}=x \boldsymbol{s} y-(-1)^{(|x|+1)(|y|+1)} y \mathbf{k} x
$$

which are interchanged by the involution $\mathcal{T}$. The proof that the construction in $\$ \mathrm{D} .4$ agrees with the formula (14.1.2) is essentially the same as the proof of Corollary 5.3 in [CS]. The proof of $(\mathrm{A}),(\mathrm{B}),(\mathrm{C})$ in Theorem 14.2 14.1 is then similar to the argument in [CS] $\S 4$. 


\section{Appendix E. Associativity of $\circledast$}

E.1. In this section we complete the proof of Proposition 8.3. The following statements refer to the diagram "Associativity of $\circledast "$ ". We omit the parallel diagram that is obtained by taking cohomology of each of the spaces and pairs that appear in this diagram. Each mapping denoted $\tau$ denotes an inclusion with normal bundle. The corresponding homomorphism in the cohomology diagram is the Thom isomorphism. In the cohomology diagram, the squares involving arrows denoted $\tau$ commute because the relevant normal bundles pull back.

Starting with $x \times y \times z$ in the upper left corner, the product $x \circledast(y \circledast z)$ is obtained by going across the top row then down the right side of the diagram, while the product $(x \circledast y) \circledast z$ is obtained by going down the left side of the diagram and then along the bottom row. Here, the symbol $\mathcal{F}_{\frac{1}{3}}$ denotes $\phi_{\frac{1}{3}}(\mathcal{F})$ ) and the space $\mathcal{C}$ denotes the space of (three-leaf) clovers, that is, the pre-image of the (small) diagonal under the mapping

$$
\left(\mathbf{e v}_{0}, \mathbf{e v}_{\frac{1}{3}}, \mathbf{e v}_{\frac{2}{3}}\right): \Lambda \rightarrow M \times M \times M .
$$

It has a normal bundle in $\Lambda$ that is isomorphic to $T M \oplus T M$. The symbol $\mathcal{C}^{>0,>0, \geq 0}$ denotes those loops consisting of three composable loops $\alpha \cdot \beta \cdot \gamma$, with $\alpha, \beta$ glued at time $1 / 3$ and with $\beta, \gamma$ glued at time $2 / 3$, such that two (or more) of these "leaves" have positive energy (that is, one or fewer of these loops is constant). Hence $\mathcal{C}-\mathcal{C}^{>0,>0, \geq 0}$ consists of clovers such that two or more of the leaves are constant. The square marked 1 is Cartesian: the lower right corner is the intersection of the upper right and lower left corners. The symbol $\Delta$ denotes a diagonal mapping and id denotes an identity mapping.

E.2. Using the obvious extension of the notation for $\theta_{\frac{1}{2} \rightarrow s}: I \rightarrow I$ (with $\theta(0)=0$ and $\theta(1)=1)$, the mappings $J_{i}$ and $\widehat{j}_{i}$ are (re)defined by

$$
\begin{array}{ll}
J_{1}(s, \gamma)=\gamma \circ \theta_{\frac{1}{3} \rightarrow \frac{2}{3} s} & J_{2}(\gamma, t)=\gamma \circ \theta_{\frac{1}{3} \rightarrow \frac{1}{3}} \\
\frac{2}{3} \rightarrow \frac{2}{3}+\frac{2}{3} t \\
\widehat{J}_{1}(s, \gamma)=\gamma \circ \theta_{\frac{1}{3} \rightarrow s} & \widehat{J}_{2}(\gamma, t)=\gamma \circ \theta_{\frac{2}{3} \rightarrow t}
\end{array}
$$

so that

$$
\begin{aligned}
& J_{1} \circ\left(\text { id } \times \widehat{J}_{2}\right)(s, \gamma, t)=\gamma \circ \theta_{\frac{2}{3} \rightarrow t} \circ \theta_{\frac{1}{3} \rightarrow \frac{2}{3} s}=\gamma \circ \theta_{\frac{2}{3} \rightarrow \frac{1}{3}} \quad \begin{array}{r}
\frac{1}{3} \rightarrow s t \\
\frac{2}{3} \rightarrow t
\end{array} \\
& J_{2} \circ\left(\widehat{J}_{1} \times \text { id }\right)(\gamma, s, t)=\gamma \circ \theta_{\frac{1}{3} \rightarrow s} \circ \theta_{\substack{\frac{1}{3} \rightarrow \frac{1}{3} \\
\frac{2}{3} \rightarrow \frac{1}{3}+\frac{2}{3} t}}=\gamma \circ \theta_{\frac{1}{3} \rightarrow s} \underbrace{}_{\frac{2}{3} \rightarrow s+(1-s) t}
\end{aligned}
$$

We need to prove that the corresponding cohomology diagram commutes. The only part that is not obvious is the square designated 2 in the diagram. This square commutes up to (relative) homotopy for the following reason. Let $\mathfrak{m}$ denote the set of continuous nondecreasing mappings $\theta:[0,1] \rightarrow[0,1]$ such that $\theta(0)=0, \theta(1)=1$, and $\theta$ is linear on $\left[0, \frac{1}{3}\right]$, on $\left[\frac{1}{3}, \frac{2}{3}\right]$, and on $\left[\frac{2}{3}, 1\right]$. For $i=1,2,3$ let $\mathfrak{m}_{i}$ denote the collection of all $\theta \in \mathfrak{m}$ such that $\theta$ is 


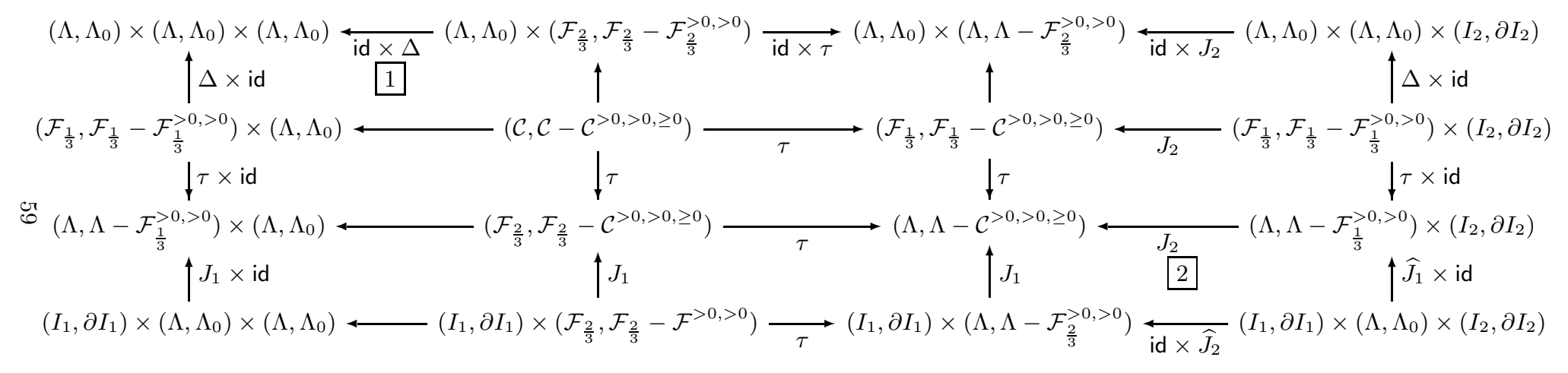

FIGURE 11 . Associativity of $\circledast$ product 
constant on $\left[\frac{i-1}{3}, \frac{i}{3}\right]$. The functions $\theta_{\text {etc. }}$ appearing on the right side of (E.2.1) and (E.2.2) may be interpreted as continuous mappings $(s, t) \in I_{1} \times I_{2} \rightarrow \mathfrak{m}$ with the following boundary behavior: $\{0\} \times I_{2} \rightarrow \mathfrak{m}_{1},\{1\} \times I_{2} \rightarrow \mathfrak{m}_{2} ; I_{1} \times\{0\} \rightarrow \mathfrak{m}_{2}$; and $I_{1} \times\{1\} \rightarrow \mathfrak{m}_{3}$. This boundary behavior is indicated in Figure 12 .

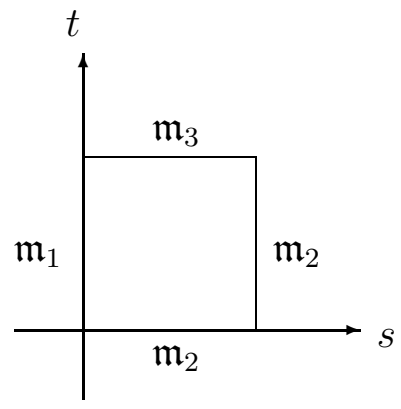

Figure 12. Boundary behavior

But the collection of such maps $I_{1} \times I_{2} \rightarrow \mathfrak{m}$ is convex, so the mappings (E.2.1) and (E.2.2) are homotopic. This completes the proof that the $\circledast$ product is associative.

\section{References}

[AS1] B. Abbondandolo and M. Schwarz, Notes on Floer homology and loop space homology, in Morse theoretic methods in nonlinear analysis and in symplectic topology, Proceedings of the NATO Advanced Study Institute, Montral, Canada, July 2004, Springer, Dordrecht, 2006.

[AS2] B. Abbondandolo and M. Schwarz, On the Floer homology of cotangent bundles, Comm. Pure. Appl. Math. 59 (2005), 254-316.

[A] D. Anosov, Certain homotopies in the space of closed curves. Math. USSR Izvestiya 17 (1981), 423-453.

[Ba] N. Baas, On bordism theories of manifolds with singularities, Math. Scand.,33 (1973), 279-302.

[BC] A. Bahri and F. R. Cohen, On "small geodesics" and free loop spaces, preprint/work in progress

[BO] A. Baker and C. Özel, Complex cobordism of Hilbert manifolds with some applications to flag varieties and loop groups. in Geometry and Topology: Aarhus 1998,1-19, Contemp. Math. 258, Amer. Math. Soc., Providence RI, 2000.

[Bal] W. Ballman, G. Thorbergsson and W. Ziller, Closed geodesics on positively curved manifolds, Ann. Math. 116 (1982), 213-247.

[Ban] V. Bangert, On the existence of closed geodesics on two-spheres, Int. J. Math. 4 (1993), 1-10.

[Be] A. Besse, Manifolds all of whose Geodesics are Closed, Ergebnisse der Mathematik 93, Springer-Verlag, Berlin, 1978.

[BH1] G. Birkhoff and M. Hestenes, Generalized minimax principle in the calculus of variations, Proc Natl Acad Sci U.S.A. 1935 February; 21(2): 96-99.

[BH2] G. Birkhoff and M. Hestenes, Generalized minimax principle in the calculus of variations, Duke Math. J. 1, no. 4 (1935), 413-432

[BM] A. Borel and J. C. Moore, Homology theory for locally compact spaces, Michigan Math. J. 7, (1960), $137-159$. 
[Bo1] R. Bott, On the iteration of closed geodesics and the Sturm intersection theory, Comm. Pure Appl. Math. 9 (1956), 171-206.

[Bo2] R. Bott, Morse theory and its application to homotopy theory, Lectures delivered in Bonn, 1958, notes by A. van de Ven. Harvard lecture notes; Reprinted in Collected Papers, Vol. 1-4, Birkhäuser, Boston, 1994, 1995.

[Br] G. Bredon, Sheaf Theory (second edition), Graduate Texts in Mathematics 170, Springer Verlag, N. Y., 1997.

[Br2] G. Bredon, Topology and Geometry, Springer Verlag, New York, 1993.

[BRS] S. Buoncristiano, C. Rourke and B. Sanderson, A geometric approach to homology theory, L. M. S. Lecture Notes 18, Cambridge Univ. Pr., Cambridge, 1978.

[C] K. -C. Chang, Infinite Dimensional Morse Theory and Multiple Solution Problems, PNLDE 6, Birkhäuser Boston, 1993.

[Chs] M. Chas, Combinatorial Lie bialgebras of curves on surfaces, Topology 43 (2004), 543-568.

[CS] M. Chas and D. Sullivan, String topology, preprint, math.GT/9911159 (1999).

[Cht] D. Chataur, A bordism approach to string topology, preprint, math.AT/0306080.

[Co] R. Cohen, Homotopy and geometric perspectives on string topology, Lecture notes, Stanford University, 2005.

[CHV] R. Cohen, K. Hess, and A. Voronov, String Topology and Cyclic Homology, Birkhauser, Basel, 2006.

[CJ] R. Cohen and J. Jones, A homotopy theoretic realization of string topology, Math. Ann. 324 (2002), 773-798.

[CJY] R. Cohen, J. Jones, and J. Yan, The loop homology algebra of spheres and projective spaces, Progr. Math. 215, Birkhauser, Basel 2003, 77-92.

[CKS] R. Cohen, J. Klein, and D. Sullivan, The homotopy invariance of the string topology loop product and string bracket, math.GT/0509667.

[D] A. Dold, Lectures on Algebraic Topology, Grundlehren Math. 200, Springer Verlag, Berlin, 1972.

[ES] S. Eilenberg and N. Steenrod, Foundations of Algebraic Topology, Princeton University Press, 1952, Princeton, NJ.

[F] J. Franks, Geodesics on $S^{2}$ and periodic points of annulus homeomorphisms, Invent. Math. 108 (1992), 403-418.

$[\mathrm{GeM}]$ S. I. Gelfand and Yu. I. Manin, Methods of Homological Algebra, second edition, Springer Verlag, Berlin, 2002.

[GeM2] S. I. Gelfand and Yu. I. Manin, Homological Algebra, Encyclopedia of Mathematics 38, Algebra V, Springer Verlag, Berlin, 1994.

[Go] W. Goldman, Invariant functions on Lie groups and Hamiltonian flows of surface group representations, Inv. Math. 85 (1986), 263-302.

[Gor] M. Goresky, Triangulation of stratified objects, Proc. Amer. Math. Soc. 72 (1978), 193-200.

[GoM] M. Goresky and R. MacPherson, Intersection Homology II, Inv. Math. 71 (1983), 77-129.

[GrM] D. Gromoll and W. Meyer, Periodic geodesics on compact Riemannian manifolds, J. Diff. Geom. 3 (1969), 493-510.

[Har] R. Hardt, Stratification of real analytic mappings and images. Invent. Math. 28 (1975), 193-208.

[Ha] A. Hatcher, Algebraic Topology, Cambridge University Press, Cambridge, UK, 2002.

[Hi1] N. Hingston, On the lengths of closed geodesics on a two-sphere, Proc. Amer. Math. Soc. 125 (1997), 3099-3106. 
[Hi2] N. Hingston, On the growth of the number of closed geodesics on the two-sphere, Int. Math. Res. Not. 9 (1993), 253-262.

[Hir] H. Hironaka, Subanalytic sets. Number theory, algebraic geometry and commutative algebra, in honor of Yasuo Akizuki, pp. 453-493. Kinokuniya, Tokyo, 1973.

[Hs] M. Hirsch, On normal microbundles, Topology 5 (1966), 229-240.

[Ho] P. Holm, Microbundles and Thom classes, Bull. Amer. Math. Soc. 72 (1966), 549-554.

[I] B. Iverson, Cohomology of Sheaves, Universitext, Springer Verlag, Berlin, 1986.

[J] F. E. A. Johnson, On the triangulation of stratified sets and singular varieties, Trans. Amer. Math. Soc. 275 (1983), 333-343.

$[\mathrm{K}] \quad$ W. Klingenberg, Lectures on Closed Geodesics, Grundlehren der mathematischen Wissenschaften 230, Springer Verlag, Berlin, 1978.

[L] Y. Long, Index Theory for Symplectic Paths with Applications Progress in Mathematics 207, Birkhäuser, Basel, 2002.

[Mi] J. Milnor, Morse Theory, Annals of Mathematics Studies 51, Princeton University Press, Princeton N.J., 1963.

[Mi2] J. Milnor, Microbundles, Part I, Topology 3, supp. 1 (1964), 53-80.

[Mo1] M. Morse, Calculus of Variations in the Large, Amer. Math. Soc. Colloquium Publications, XVIII, Providence, R.I., 1934.

[Mo2] M. Morse, Functional Topology and Abstract Variational Theory, Memoriale des Sciences Math. 92, Gauthier-Villars, Paris, 1939.

[P] D. Pope, On the approximation of function spaces in the calculus of variations, Pacific J. Math. ? (1962), 1029-1045.

[R] H. -B. Rademacher, On the average indices of closed geodesics, J. Diff. Geom. 29 (1989), 65-83.

[SaW] D. Salamon and J. Weber, Floer homology and the heat flow, Geometric and Functional Analysis (GAFA) 16 (2005), 1050-1138.

[Sp] E. Spanier, Algebraic Topology, McGraw-Hill, New York, 1966.

[Su] D. Sullivan, Open and closed string field theory interpreted in classical algebraic topology, in Topology, Geometry and Quantum Field Theory, Procdeedings of the 2002 Oxford Symposium, London Mathematical Society Lecture Note Series 308, Cambridge University Press, Cambridge, 2004; pages 344-357.

[T] V. Turaev, Skein quantization of Poisson algebras of loops on surfaces, Ann. Sci. École Norm. Sup. 24 (1991), 635-704.

[V] J. L. Verdier, Des Catégories Dérivées des Catégories Abéliennes, 1963, reprinted in Astérisque 239, Soc. Math. de France, Paris, 1996.

[Vi] C. Viterbo, Functors and computations in Floer homology with applications II, preprint, 1996, revised 2003.

[VS] M. Vigué-Poirrier and D. Sullivan, The homology theory of the closed geodesic problem, J. Diff. Geom. 11 (1976), 633-644.

[Wh] G. Whitehead, Generalized Homology Theories, Trans. Amer. Math. Soc. 102 (Feb., 1962), pp. 227-283.

[Wi] B. Wilking, Index parity of closed geodesics and rigidity of Hopf fibrations, Inv. Math. 144 (2001), 281-295.

[Wo] S. Wolpert, On the symplectic geometry of deformations of hyperbolic surfaces, Ann. Math. 117 (1983), 207-234.

[Z] W. Ziller, Geometry of the Katok examples. Ergod.Th. \& Dyn.Syst. 3 (1982) 